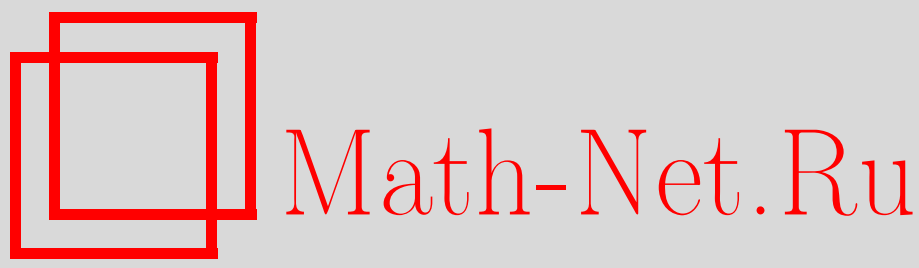

В. В. Жиков, Усреднение задач теории упругости на сингулярных структурах, Изв. РАН. Сер. матем., 2002, том 66, выпуск 2, 81-148

DOI: https://doi.org/10.4213/im380

Использование Общероссийского математического портала Math-Net.Ru подразумевает, что вы прочитали и согласны с пользовательским соглашением http://www. mathnet.ru/rus/agreement

Параметры загрузки:

IP : 3.85 .5 .30

26 апреля 2023 г., 13:38:12 
УДК 517.9

\author{
В. В. Жииков
}

\title{
Усреднение задач теории упругости на сингулярных структурах
}

\begin{abstract}
Рассмотрена теория усреднения на периодических сетках, сочленениях плит и стержней, а также вообще на сингулярных объектах. Установлено, что в типичном случае усредненное уравнение имеет "неклассический" характер. Этот факт существенно отличает усреднение задач теории упругости от усреднения скалярных задач.

Проведено исследование свойств соболевских пространств для различных сингулярных структур, доказан неклассический принцип усреднения для сингулярных периодических структур общего вида, установлен "масштабный эффект" для модельных задач, содержащих два малых геометрических параметра.

Библиограффия: 17 наименований.
\end{abstract}

\section{$\S 1$. Введение}

1.1. Простейший сингулярный объект - это отрезок (стержень) на плоскости, рассматриваемый с точки зрения двумерной теории упругости. На рис. 1 представлены другие примеры сингулярных объектов.

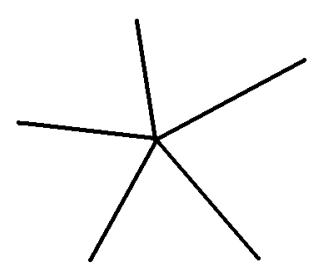

$a$

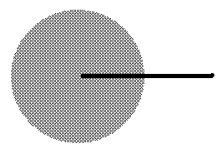

$b$

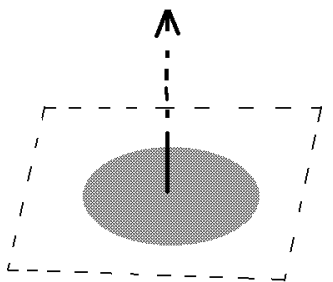

$c$

Рис. 1. $a$-пучок стержней; $b$-плита со стержнем; $c$-плита в пространстве

На каждом из указанных на рис. 1 объектов сушествует естественная мера $\mu$. Например, для плиты со стержнем (см. рис. 1,b) мера $\mu$ - это сумма плоской меры Лебега на плите и линейной меры на отрезке, для плиты в пространстве (см. рис. $1, c$ ) мера $\mu$ - это мера в $\mathbb{R}^{3}$, сосредоточенная на плите и равная на этой плите плоской мере Лебега.

Работа выполнена при финансовой поддержке РФФИ (проект № 99-01-00072).

(C) в. В. Жиков, 2002 


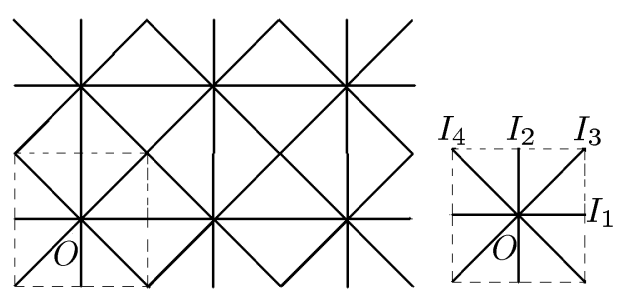

Рис. 2. Периодическая сетка

С точки зрения приложений, большой интерес представляют периодические сингулярные структуры указанного или более сложного типа. На рис. 2 представлена периодическая сетка (ячейка периодичности указана пунктиром).

Ячейка периодичности состоит из четырех отрезков: $I_{1}, I_{2}, I_{3}, I_{4}$. Меру $\mu$ определим как сумму линейных мер этих отрезков, взятых с неотрицательными весами $\rho_{1}, \rho_{2}, \rho_{3}, \rho_{4}$.

В случае $\rho_{3}=\rho_{4}=0$ имеем квадратную сетку, а в случае $\rho_{1}=1, \rho_{2}=\rho_{3}=$ $\rho_{4}=0$ - систему параллельных стержней (см. рис. 3,4$)$.
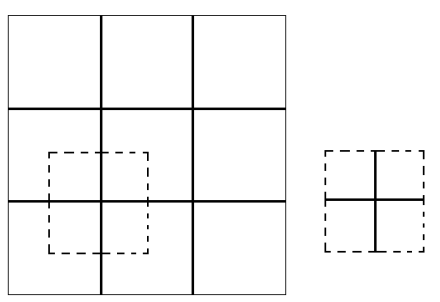

Рис. 3. Квадратная сетка

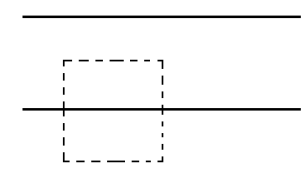

Рис. 4. Система параллельных стержней

На рис. 5 представлена периодическая составная структура. Для нее мера $\mu$ пропорциональна плоской мере Лебега на круге и линейным мерам на восьми отрезках.

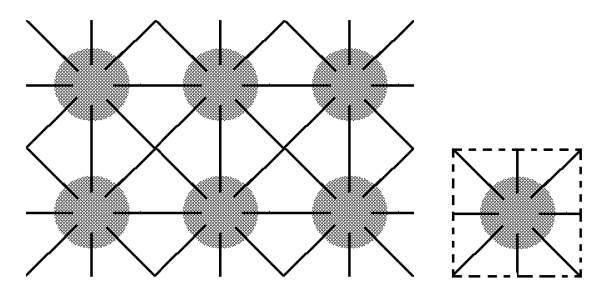

Рис. 5. Периодическая составная структура

Можно рассматривать периодические сетки в $\mathbb{R}^{3}$, а также ящичную структуру в $\mathbb{R}^{3}$. Последняя получится, если $\mathbb{R}^{3}$ разбить на единичные кубы (ячейки периодичности), а периодическую меру $\mu$ определить как плоскую лебегову меру на гранях. Перечень подобных примеров можно без труда продолжить. 
В общем случае сингулярньй периодический объект описывается периодической борелевской мерой $\mu, \square=[0,1)^{N}$ - ячейка периодичности, $\int_{\square} d \mu=1$. При этом сама сингулярная структура - это просто носитель меры $\mu$. Обычно носитель есть объединение некоторых многообразий разной размерности, и на каждом таком многообразии мера $\mu$ пропорциональна соответствующей мере Лебега.

Для постановки задачи усреднения введем меру $\mu_{\varepsilon}$ равенством

$$
\mu_{\varepsilon}(B)=\varepsilon^{N} \mu\left(\varepsilon^{-1} B\right)
$$

для любого борелевского множества $B \subset \mathbb{R}^{N}$. Мера $\mu_{\varepsilon}$ имеет период $\varepsilon$, и справедливо равенство

$$
\int_{\varepsilon \square} d \mu_{\varepsilon}=\varepsilon^{N} \int_{\square} d \mu=\varepsilon^{N}
$$

Отсюда следует, что мера $\mu_{\varepsilon}$ слабо сходится к мере Лебега: $d \mu_{\varepsilon} \rightarrow d x$, т.е.

$$
\lim _{\varepsilon \rightarrow 0} \int_{\mathbb{R}^{N}} \varphi d \mu_{\varepsilon}=\int_{\mathbb{R}^{N}} \varphi d x, \quad \varphi \in C_{0}^{\infty}\left(\mathbb{R}^{N}\right) .
$$

Пусть $\Omega$ - ограниченная липшицева область в $\mathbb{R}^{N}$. С сингулярньм объектом $\left(\Omega, d \mu_{\varepsilon}\right)$ мы должны связать некоторую задачу теории упругости. Прежде всего следует определить отвечающее теории упругости соболевское пространство $\mathcal{H}_{0}\left(\Omega, d \mu_{\varepsilon}\right)$.

Для гладкого вектора $u=\left(u^{1}, u^{2}, \ldots, u^{N}\right)$ определим тензор деформации

$$
e(u)=\frac{1}{2}\left(\frac{\partial u^{i}}{\partial x_{j}}+\frac{\partial u^{j}}{\partial x_{i}}\right)
$$

Пусть $W=W_{\varepsilon}$ - замыкание множества пар $\left\{u, e(u): u \in C_{0}^{\infty}(\Omega)^{N}\right\}$ в произведении пространств $L^{2}\left(\Omega, d \mu_{\varepsilon}\right)^{N} \times L^{2}\left(\Omega, d \mu_{\varepsilon}\right)^{\frac{N(N+1)}{2}}$. Элементами $W$ служат пары $u, z$, где $u$ - вектор, а $z$ - симметрическая матрица. Условимся матрицу $z$ обозначать $e(u)$ и называть симметрическим градиентом вектора и. Совокупность первых компонент $u$ образует пространство $\mathcal{H}_{0}^{1}\left(\Omega, d \mu_{\varepsilon}\right)$. При таком определении симметрический градиент не единствен, но это пока несушественно.

Через $A=\left\{a_{i j s h}\right\}$ обозначим тензор упругости, подчиненный обычным условиям симметрии: $a_{i j s h}=a_{s h i j}=a_{j i s h}$.

Пусть $\xi=\left\{\xi_{i j}\right\}$ - симметрическая матрица. Скалярное произведение матриц определим по обычному правилу: $\xi \cdot \eta=\xi_{i j} \eta_{i j}$, в частности $\xi^{2}=\xi_{i j} \xi_{i j}$. Для тензора упругости $A$ определим его действие на матрицу $\xi$ по правилу $A \xi=\left\{a_{i j s h} \xi_{s h}\right\}$. Тогда $A \xi \cdot \xi=a_{i j s h} \xi_{i j} \xi_{s h}$ - плотность упругой энергии. Предполагается, что выполнено условие положительной определенности

$$
\nu \xi^{2} \leqslant A \xi \cdot \xi \leqslant \nu^{-1} \xi^{2}, \quad \nu>0
$$

Изучим следующую модельную задачу усреднения:

$$
\begin{gathered}
u_{\varepsilon} \in \mathcal{H}_{0}^{1}\left(\Omega, d \mu_{\varepsilon}\right), \quad-\operatorname{div}\left(A_{\varepsilon} e\left(u_{\varepsilon}\right)\right)+u_{\varepsilon}=f_{\varepsilon}, \\
A_{\varepsilon}=A_{\varepsilon}(x)=A\left(\varepsilon^{-1} x\right)
\end{gathered}
$$


где $A(y)$ - периодический $\mu$-измеримьй тензор упругости, подчиненный условию коэрцитивности $(1.2), f_{\varepsilon} \in L^{2}\left(\Omega, d \mu_{\varepsilon}\right)^{N}$.

По определению $u_{\varepsilon} \in \mathcal{H}_{0}^{1}\left(\Omega, d \mu_{\varepsilon}\right)$ - решение уравнения (1.3), если выполнено интегральное тождество

$$
\int_{\Omega}\left[A_{\varepsilon} e\left(u_{\varepsilon}\right) \cdot e(\psi)+u_{\varepsilon} \cdot \psi\right] d \mu_{\varepsilon}=\int_{\Omega} f_{\varepsilon} \cdot \psi d \mu_{\varepsilon}, \quad \psi \in C_{0}^{\infty}(\Omega)^{N}
$$

в котором $e\left(u_{\varepsilon}\right)$ - некоторый симметрический градиент $u_{\varepsilon}$. Сушествование и единственность решения в виде пары $u_{\varepsilon}, e\left(u_{\varepsilon}\right)$ следует из теоремы Рисса о представлении (см. $\S 5)$.

В дальнейшем подробно изучим соболевские пространства, отвечающие задачам теории упругости на сингулярных объектах. Здесь только отметим, что для квадратной сетки пространство $\mathcal{H}_{0}\left(\Omega, d \mu_{\varepsilon}\right)$ имеет тривиальную структуру: распадается на пространства, отвечаюшие отдельным стержням. В связи с этим и сами задачи теории упругости на таких сетках тривиальны. Однако для более сложных структур, когда в узлах пересекаются три или более стержней, соболевские пространства и соответствуюшие задачи уже весьма сложны.

Цель усреднения в том, что решение $u_{\varepsilon}$ задачи (1.3) в определенном смысле сходится к решению усредненной задачи, связанной уже с мерой Лебега, поскольку $d \mu_{\varepsilon} \rightarrow d x$. Здесь требуется пояснить, что означает сама сходимость последовательности функций $v_{\varepsilon} \in L^{2}\left(\Omega, d \mu_{\varepsilon}\right)$ к функции $v \in L^{2}(\Omega, d x)=L^{2}(\Omega)$. В связи с этим напомним понятие сходимости "в переменном пространстве $L^{2}$ ".

Будем считать, что последовательность $v_{\varepsilon}$ ограничена в $L^{2}\left(\Omega, d \mu_{\varepsilon}\right)$, т.е.

$$
\limsup _{\varepsilon \rightarrow 0} \int_{\Omega}\left|v_{\varepsilon}\right|^{2} d \mu_{\varepsilon}<\infty
$$

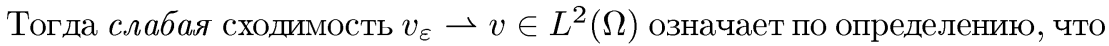

$$
\lim _{\varepsilon \rightarrow 0} \int_{\Omega} \varphi v_{\varepsilon} d \mu_{\varepsilon}=\int_{\Omega} \varphi v d x \quad \forall \varphi \in C_{0}^{\infty}(\Omega)
$$

Сильная сходимость $v_{\varepsilon} \longrightarrow v$ означает, что

$$
\lim _{\varepsilon \rightarrow 0} \int_{\Omega} v_{\varepsilon} z_{\varepsilon} d \mu_{\varepsilon}=\int_{\Omega} v z d x, \quad \text { как только } z_{\varepsilon} \rightarrow z
$$

ПРЕДЛОЖЕНИЕ 1.1. Справедливы следующие утверждения:

(i) всякая ограниченная последовательность компактна в смысле слабой сходимости;

(ii) если $v$-достаточно гладкая функиия, например $v \in C(\bar{\Omega})$, то

$$
v_{\varepsilon} \rightarrow v \Longleftrightarrow \lim _{\varepsilon \rightarrow 0} \int_{\Omega}\left|v-v_{\varepsilon}\right|^{2} d \mu_{\varepsilon}=0
$$


1.2. Рассмотрим множество $\mathcal{R}$ периодических жестких перемещений. По определению

$$
u \in \mathcal{R} \Longleftrightarrow \exists \varphi_{n} \in C_{\mathrm{per}}^{\infty}(\square)^{N}: \varphi_{n} \rightarrow u, \quad e\left(\varphi_{n}\right) \rightarrow 0 \quad \text { в } L^{2}(\square, d \mu),
$$

где $C_{\mathrm{per}}^{\infty}-$ множество всех бесконечно дифференцируемых периодических функций на $\mathbb{R}^{N}$.

Постоянные векторы, очевидно, всегда принадлежат $\mathcal{R}$, но обычно существуют нетривиальные жесткие перемещения. Для периодической сетки это будут все векторы из $L^{2}(\square, d \mu)^{2}$, которые на каждом ребре ортогональны этому ребру (трансверсальные векторы, или поперечные перемещения).

Приведем упрошенную формулировку принципа усреднения для задач теории упругости. В ней подчеркнем только роль нетривиальных периодических жестких перемешений, оставив без внимания другие обстоятельства.

С периодическим тензором $A=A(y)$ свяжем усредненный тензор по правилу

$$
A^{\text {hom }} \xi \cdot \xi=\inf _{w \in C_{\text {per }}^{\infty}(\square)^{N}} \int_{\square} A(y)(\xi+e(w(y))) \cdot(\xi+e(w(y))) d \mu(y) .
$$

ПРИНЦИП УСРЕДНЕНИЯ. Пусть $u_{\varepsilon}-$ решение задачи Дирихле (1.3), причем $f_{\varepsilon}=f \in C^{\infty}(\bar{\Omega})^{N}$. Тогда имеет место слабая сходимость

$$
u_{\varepsilon} \rightarrow T u_{0}+(E-T) f
$$

где $T: \mathbb{R}^{N} \rightarrow \mathbb{R}^{N}$ - некоторый линейный неотрицательный оператор, связанный с существованием нетривиальных периодических жсестких перемещений; в частности, $T=E$ (единичный оператор), если таковые отсутствуют. При этом $u_{0}-$ решение задачи Дирихле:

$$
u_{0} \in H_{0}^{1}(\Omega)^{N}, \quad-\operatorname{div} A^{\mathrm{hom}} e\left(u_{0}\right)+T u_{0}=T f .
$$

В случае $T=$ Е сходимость в (1.8) становится сильной, а уравнение (1.9) классическим усредненным уравнением:

$$
\begin{gathered}
\int_{\Omega}\left|u_{\varepsilon}(x)-u_{0}(x)\right|^{2} d \mu_{\varepsilon} \rightarrow 0, \\
u_{0} \in H_{0}^{1}(\Omega)^{N}, \quad-\operatorname{div} A^{\text {hom }} e\left(u_{0}\right)+u_{0}=f .
\end{gathered}
$$

Оператор $T$ обычно нетрудно вычислить в явном виде (см. далее $(2.8))$.

Для иллюстрации принципа усреднения приведем два примера.

$1^{\circ}$. Усреднение в перфорированных областях. Рассмотрим периодическую связную область $F$ в $\mathbb{R}^{N}$ и соответствующую ей меру

$$
d \mu=\rho(x) d x, \quad \rho(x)=\left\{\begin{array}{cll}
\frac{1}{|\square \cap F|}, & \text { если } & x \in F, \\
0, & \text { если } & x \in \mathbb{R}^{N} \backslash F .
\end{array}\right.
$$

В этом случае $d \mu_{\varepsilon}=\rho\left(\varepsilon^{-1} x\right) d x$ и интегрирование в (1.4) ведется только по "перфорированной" области $\Omega_{\varepsilon}=\Omega \cap F_{\varepsilon}$, где $F_{\varepsilon}=\varepsilon F$. Получаем задачу в области $\Omega_{\varepsilon}$, когда на $\partial \Omega \cap \partial F_{\varepsilon}$ задается условие Дирихле $u_{\varepsilon}=0$, а на остальной 
части гранищы области $\Omega_{\varepsilon}-$ естественное краевое условие $A e\left(u_{\varepsilon}\right) n=0$, где $n-$ единичная нормаль к границе.

Из связности множества $F$ в $\mathbb{R}^{N}$ ясно, что периодические жесткие перемещения - только константы. Поэтому $T=E$ и получено классическое усреднение (1.10). При этом на периодическое множество $F$ никаких требований гладкости, например липшицевости, не накладывается. Отметим, что в многочисленных работах по перфорированным областям (см. [1]-[6]) применялись другие методы и требование гладкости множества $F$ было обязательным.

Рассмотренный пример является исключением. Во всех остальных случаях множество $\mathcal{R}$ нетривиально, нетривиален и оператор $T$.

$2^{\circ}$. Периодическая сетка. Здесь оператор $T$ нетривиален,

$$
T=\frac{1}{2}\left(\begin{array}{cc}
1+\rho_{1}-\rho_{2} & \rho_{3}-\rho_{4} \\
\rho_{3}-\rho_{4} & 1-\rho_{1}+\rho_{2}
\end{array}\right)
$$

а усредненный тензор $A^{\text {hom }}$ можно вычислить в явной форме. Ограничимся рассмотрением случая, когда исходный тензор $A$ постоянен и изотропен:

$$
\begin{aligned}
& A \xi=k \xi+k_{1} E \operatorname{tr} \xi, \quad k>0, \quad k_{1} \geqslant 0, \quad \xi=\left(\begin{array}{ll}
\xi_{11} & \xi_{12} \\
\xi_{12} & \xi_{22}
\end{array}\right), \\
& \operatorname{tr} \xi=\xi_{11}+\xi_{22}, \quad E=\left(\begin{array}{ll}
1 & 0 \\
0 & 1
\end{array}\right), \quad A \xi \cdot \xi=k \xi^{2}+k_{1}(\operatorname{tr} \xi)^{2} .
\end{aligned}
$$

Тогда

$$
\begin{gathered}
A^{\text {hom }} \xi \cdot \xi=\hat{k}\left[\rho_{1} \xi_{11}^{2}+\rho_{2} \xi_{22}^{2}+\frac{1}{4} \rho_{3}\left(\xi_{11}+2 \xi_{12}+\xi_{22}\right)^{2}\right. \\
\left.+\frac{1}{4} \rho_{4}\left(\xi_{11}-2 \xi_{12}+\xi_{22}\right)^{2}\right] \\
\hat{k}=k \frac{k+2 k_{1}}{k+k_{1}} .
\end{gathered}
$$

1.3. Необходимо отметить, что для скалярных задач (когда тензор деформации $e(u)$ заменяется градиентом $\nabla u$ скалярной функции) проблема усреднения более простая и определенная. Здесь при весьма общих условиях имеет место классический принцип усреднения вида (1.10). Единственное требование - это эргодичность меры $\mu$ (см. [7], [8]). Напомним, что мера $\mu$ әргодична или 2-связна, если $u \equiv$ const $\mu$-п.в., как только найдутся $u_{n} \in C_{\mathrm{per}}^{\infty}(\square)$ такие, что $u_{n} \longrightarrow u, \nabla u_{n} \rightarrow 0$ в $L^{2}(\square, d \mu)$.

Более формально, эргодичность меры $\mu$ означает, что функция $u$ есть константа, если она принадлежит соболевскому пространству периодических функций $H_{\mathrm{per}}^{1}(\square, d \mu)$ и имеет нулевой градиент. Во всех примерах, которые рассматриваются или упоминаются в настоящей работе, соответствующая мера $\mu$ эргодична.

Для задач теории упругости понятие эргодичности неприменимо, поскольку рассматриваются не градиенты, а тензоры деформации. Равенство нулю тензора деформации в соболевском пространстве $\mathcal{H}_{\mathrm{per}}^{1}(\square, d \mu)$ означает, что $u$ - периодическое жесткое перемешение, которое может не быть постоянным вектором. Самым примечательным следствием этого служит тот факт, что последовательность 
решений $u_{\varepsilon}$ задачи (1.3) не является компактной в смысле сильной сходимости в $L^{2}\left(\Omega, d \mu_{\varepsilon}\right)$. В физических терминах это значит, что решение $u_{\varepsilon}$ "осциллирует" (в отличие от классического усреднения, где имеется сильная сходимость).

Естественно ожидать, что осциллируюшая компонента решения должна описываться с помощью нетривиального жесткого периодического перемещения. Действительно, оказывается, можно найти корректирующую добавку $\chi=\chi(x, y)$ такую, что $\chi(x, \cdot)$ - нетривиальное периодическое жесткое перемещение, а разность

$$
u_{\varepsilon}(x)-u_{0}(x)-\chi\left(x, \frac{x}{\varepsilon}\right)
$$

уже сильно сходится к нулю. При этом "предельной функцией" естественно считать функцию $u(x, y)=u_{0}(x)+\chi(x, y)$ двух переменных $x \in \Omega$ и $y \in \square$. Эта функция служит “двухмасштабным пределом" последовательности $u_{\varepsilon}$.

\section{§ 2. Неклассический принцип усреднения}

2.1. Множество $\mathcal{R}$ периодических жестких перемещений - это замкнутое подпространство в $L^{2}(\square, d \mu)^{N}$, содержащее постоянные векторы. Во всех примерах, которые будут подробно разобраны в дальнейшем, выполнено следуюшее условие: любой вектор $u \in \mathcal{R}$ допускает единственное представление в виде

$$
u(y)=c+g(y)
$$

где с-постоянный вектор, а $g(y)$ - трансверсальный вектор, или поперечное перемещение.

Обычно носитель меры $\mu$ - это объединение некоторых подмногообразий. Назовем вектор $g(y)$ трансверсальныц, если он отличен от нуля только на подмногообразиях размерности меньше $N$ и в каждой точке ортогонален этому подмногообразию.

Рассмотрим некоторые примеры разложения (2.1).

$1^{\circ}$. Система из одного стержня (рис. 4). В этом случае $u(y)=\left(c_{1}, 0\right)+$ $\left(0, \beta\left(y_{1}\right)\right)$, где функция $\beta\left(y_{1}\right)$ периодична по $y_{1}$.

Этот пример исключителен в двух отношениях. Во-первых, разложение (2.1) ортогонально в $L^{2}(\square, d \mu)^{2}$; во-вторых, постоянный вектор $c$ из (2.1) пробегает одномерное подпространство из $\mathbb{R}^{2}$. Для более сложных структур ничего подобного не наблюдается.

$2^{\circ}$. Квадратная сетка (рис. 3). Здесь $u(x)=\left(c_{1}, c_{2}\right)+g(y), g$-трансверсальньй вектор (см. рис. 6).

Это разложение неортогонально, а $c$ пробегает все пространство $\mathbb{R}^{2}$.

$3^{\circ}$. Периодическая составная структура (рис. 5). В этом случае $c$ пробегает все пространство $\mathbb{R}^{2}$, а $g$ - произвольньй трансверсальньй вектор, отличный от нуля вне плиты $P$, т.е. $\left.g\right|_{P}=0$ в $L^{2}(P, d \mu)$ (не только в $\left.L^{2}(P, d x)\right)$.

Таким образом, за исключением случая одного стержня, разложение (2.1) принимает вид

$$
\mathcal{R}=\mathbb{R}^{N}+\mathcal{R}_{1},
$$

где $\mathcal{R}_{1}$ - множество всех трансверсальных или поперечных жестких перемешений. 


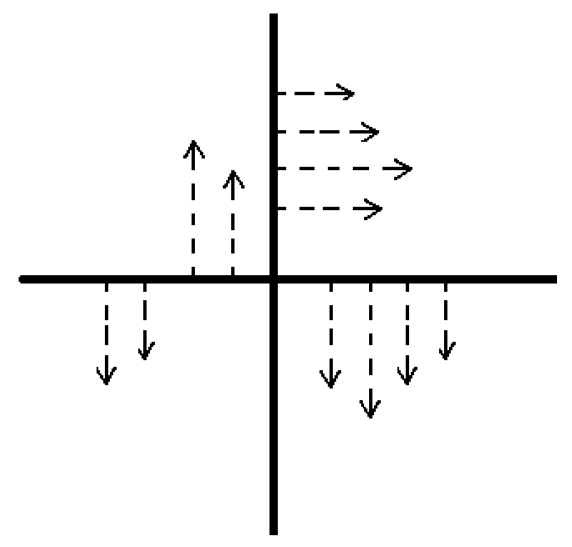

Рис. 6. Поперечные перемешения на сетке

Аналог разложений (2.1), (2.2) имеет смысл и в случае меры $\mu$ общего вида. Для этого требуется подходяшим образом сформулировать понятие "поперечного жесткого перемещения" (общую формулировку см. в $§ 7$ ).

2.2. Для некоторого упрошения формулировок будем сначала считать, что усредненный тензор положительно определенный:

$$
A^{\mathrm{hom}} \xi \cdot \xi \geqslant c_{0} \xi^{2}, \quad c_{0}>0
$$

Например, для периодической сетки явная формула (1.12) дает положительную определенность усредненного тензора, если три веса из четырех $\rho_{1}, \rho_{2}, \rho_{3}, \rho_{4}$ положительны. Все результаты сохраняются и для вырожденного тензора $A^{\text {hom }}$ (это будет видно в дальнейшем).

НЕКЛАССИЧЕСКИЙ ПРИНЦИП УСРЕДНЕНИЯ. Пусть $u_{\varepsilon}(x)$ - решение исходной задачи (1.3), причем $f_{\varepsilon}(x)=f(x) \in C^{\infty}(\bar{\Omega})^{N}$. Тогда найдется векторфункиия $и(x, y)$ двух переменных $x \in \Omega, y \in \mathbb{R}^{N}, u(x, y) \in L^{2}(\Omega \times \square, d x \times d \mu)^{N}$, периодическая по у и такая, что

$$
\lim _{\varepsilon \rightarrow 0} \int_{\Omega}\left|u_{\varepsilon}(x)-u\left(x, \varepsilon^{-1} x\right)\right|^{2} d \mu_{\varepsilon}=0 .
$$

Вектор-функщия $и(x, \cdot)$ - это периодическое жсесткое перемещение, и в соответствии с разложением (2.1) (см. также (2.2))

$$
u(x, y)=u_{0}(x)+\chi(x, y), \quad \chi(x, \cdot) \in \mathcal{R}_{1}
$$

При этом выполняются соотношения

$$
\begin{gathered}
u_{0} \in H_{0}^{1}(\Omega)^{N}, \quad-\operatorname{div} A^{\mathrm{hom}} e\left(u_{0}\right)+\langle u\rangle=f, \\
u(x, \cdot)-f(x) \perp \mathcal{R}_{1}
\end{gathered}
$$


əде $\langle u\rangle=\int_{\square} u(x, y) d \mu(y)=u_{0}(x)+\int_{\square} \chi(x, y) d \mu(y)$.

Покажем, что соотношения (2.5)-(2.7) однозначно определяют векторфункцию $u$, и поэтому их следует рассматривать как одно предельное или усредненное уравнение. Действительно, рассмотрим ортогональные проекторы

$$
P_{1}: \mathcal{R} \rightarrow \mathcal{R}_{1}, \quad P_{2}=I-P_{1}
$$

Тогда из (2.5), (2.7) имеем

$$
\begin{gathered}
P_{1} u_{0}+\chi=P_{1} f \\
u=u_{0}+\chi=P_{2} u_{0}+P_{1} f, \quad\langle u\rangle=\left\langle P_{2} u_{0}\right\rangle+\left\langle P_{1} f\right\rangle .
\end{gathered}
$$

Определив оператор $T: \mathbb{R}^{N} \rightarrow \mathbb{R}^{N}$ равенством

$$
T c=\left\langle P_{2} c\right\rangle, \quad c \in \mathbb{R}^{N}
$$

получим два соотношения

$$
\begin{gathered}
u_{0} \in H_{0}^{1}(\Omega)^{N}, \quad-\operatorname{div} A^{\mathrm{hom}} e\left(u_{0}\right)+T u_{0}=T f \\
P_{1} u_{0}+\chi=P_{1} f
\end{gathered}
$$

Первое соотношение представляет отдельную эллиптическую задачу для слагаемого $u_{0}$, а второе - связь между слагаемыми $u_{0}$ и $\chi$.

Отметим, что проектор $P_{1}: \mathcal{R} \rightarrow \mathcal{R}_{1}$ действует в пространстве периодических функций, т.е. в выражении $P_{1} u_{0}$ вектор $u_{0}=u_{0}(x)$ рассматривается как постоянньй. В каждом конкретном примере легко находим ортогональное разложение постоянного вектора: $c=P_{1} c+P_{2} c$. Обе проекции - это периодические функции, а не постоянные векторы (за исключением случая системы из одного стержня).

2.3. Дадим более формальное описание неклассической усредненной задачи. Для этого введем множество

$$
V: \varphi=\varphi(x, y)=\varphi_{0}(x)+\varphi_{1}(x, y), \quad \varphi_{0} \in H_{0}^{1}(\Omega)^{N}, \quad \varphi_{1} \in L^{2}\left(\Omega, \mathcal{R}_{1}\right) .
$$

ОПРЕДЕЛЕНИЕ 2.1. НаЗовем $u=u_{0}(x)+\chi(x, y) \in V$ решением усредненной задачи, если интегральное тождество

$$
\int_{\Omega} A^{\text {hom }} e\left(u_{0}\right) \cdot e\left(\varphi_{0}\right) d x+\int_{\Omega} \int_{\square} u \cdot \varphi d x d \mu=\int_{\Omega} \int_{\square} f \cdot \varphi d x d \mu
$$

выполнено для любой вектор-функции $\varphi=\varphi(x, y)=\varphi_{0}(x)+\varphi_{1}(x, y) \in V$.

Левая часть равенства (2.10) задает на $V$ скалярное произведение, причем само множество $V$ полно относительно соответствущей нормы. Поэтому сушествование и единственность решения очевидны. Далее, взяв в тождестве $(2.10)$ пробную функцию $\varphi=\varphi_{0}$, а затем $\varphi=\varphi_{1}$, получим соответственно свойства (2.6) и (2.7).

Классическую усредненную задачу (1.10) можно представить в виде

$$
\mathcal{A} u_{0}+u_{0}=f \quad \text { в } L^{2}\left(\Omega, \mathbb{R}^{N}\right),
$$


где $\mathcal{A}$ - неотрицательньй самосопряженный оператор в $L^{2}\left(\Omega, \mathbb{R}^{N}\right)$, определенньй квадратичной формой

$$
\int_{\Omega} A^{\mathrm{hom}} e\left(u_{0}\right) \cdot e\left(u_{0}\right) d x, \quad u_{0} \in \mathcal{H}_{0}^{1}(\Omega) .
$$

Легко видеть, что и неклассическая задача (2.10) имеет аналогичную структуру $\mathcal{A} u+u=f$, но теперь $\mathcal{A}$ - неотрицательный самосопряженный оператор в $L^{2}(\Omega, \mathcal{R})$, определенный квадратичной формой

$$
\mathbf{a}(u, u)=\int_{\Omega} A^{\mathrm{hom}} e\left(u_{0}\right) \cdot e\left(u_{0}\right) d x, \quad u=u_{0}+\chi \in V .
$$

Задача на собственные значения

$$
\mathcal{A} u=\lambda u, \quad u=u_{0}+\chi,
$$

сводится к двум соотношениям

$$
\begin{gathered}
u_{0} \in H_{0}^{1}(\Omega)^{N}, \quad-\operatorname{div} A^{\mathrm{hom}} e\left(u_{0}\right)=\lambda T u_{0}, \\
\lambda\left(P_{1} u_{0}+\chi\right)=0 .
\end{gathered}
$$

Первое из этих соотношений представляет отдельную спектральную задачу для слагаемого $u_{0}$, а второе - связь между слагаемыми $u_{0}$ и $\chi$. Для $\lambda=0$ оба соотношения выполнены, как только $u_{0}=0$. Другими словами, точка $\lambda=0$ является бесконечно кратным собственным значением, $L^{2}\left(\Omega, \mathcal{R}_{1}\right)$ - ядро оператора $\mathcal{A}$. Ненулевыми собственными значениями оператора $\mathcal{A}$ будут собственные значения отдельной спектральной задачи (2.11) для $u_{0}$, а соответствуюшими собственными функциями будут $u=P_{2} u_{0}$.

2.4. Ранее предполагалось, что усредненный тензор $A^{\text {hom }}$ невырожлден (cм. (2.3)). В случае вырождения этого тензора приншип усреднения сохраняется и необходимы только небольшие изменения в его формулировке. Слагаемое $u_{0}$ уже необязательно принадлежит $H_{0}^{1}(\Omega)^{N}$, но $A^{\text {hom }} e\left(u_{0}\right) \in L^{2}(\Omega)$ в смысле распределений. Более того, $u_{0}$ принадлежит пространству $\widetilde{\mathcal{H}}_{0}^{1}(\Omega)$, которое является замыканием $C_{0}^{\infty}(\Omega)^{N}$ по норме

$$
\left(\int_{\Omega}\left[A^{\mathrm{hom}} e(\varphi) \cdot e(\varphi)+\varphi \cdot \varphi\right] d x\right)^{\frac{1}{2}} .
$$

Предельную задачу для слагаемого $u_{0}$ теперь представим в следующем виде:

$$
u_{0} \in \tilde{\mathcal{H}}_{0}^{1}(\Omega), \quad-\operatorname{div} A^{\text {hom }} e\left(u_{0}\right)+T u_{0}=T f .
$$

Все остальные утверждения принципа усреднения остаются без изменений.

Приведем простой пример, в котором тензор $A^{\text {hom }}$ вырожден. Рассмотрим периодическую систему горизонтальных стержней. Проверим, что в этом исключительном случае неклассическое усреднение совпадает с классическим. Разложение (2.1) ортогонально,

$$
\begin{gathered}
u_{0}=\left(u_{0}^{1}, 0\right), \quad c=P_{1} c+P_{2} c=\left(0, c_{2}\right)+\left(c_{1}, 0\right), \\
T=\left(\begin{array}{ll}
1 & 0 \\
0 & 0
\end{array}\right), \quad A^{\text {hom }} \xi \cdot \xi=\hat{k} \xi_{11}^{2} .
\end{gathered}
$$


Усредненная задача для слагаемого $u_{0}$ принимает вид

$$
-\hat{k} \frac{\partial^{2} u_{0}^{1}}{\partial x_{1}^{2}}+u_{0}^{1}=f^{1}
$$

Из второго соотношения (2.9) следует, что $\chi=\left(0, f^{2}\right)$. Введем новые обозначения: $u^{1}=u_{0}^{1}, u^{2}=\chi^{2}, u=\left(u^{1}, u^{2}\right)$. Поскольку $u=u_{0}+\chi$ не зависит от $y$, то из соотношения (2.4) получим

$$
\lim _{\varepsilon \rightarrow 0} \int_{\Omega}\left|u_{\varepsilon}(x)-u(x)\right|^{2} d \mu_{\varepsilon}=0 .
$$

Следовательно,

$$
\begin{gathered}
-\hat{k} \frac{\partial^{2} u^{1}}{\partial x_{1}^{2}}+u^{1}=f^{1}, \\
u^{2}=f^{2} .
\end{gathered}
$$

Мы получили классическое усреднение.

\section{§ 3. Тонкие периодические сетки. Масштабный эффект}

3.1. Выше мы обсуждали задачу усреднения (1.3) в стандартной форме, когда имеется только один малый геометрический параметр $\varepsilon-$ период неоднородной среды. Представляют интерес задачи, содержашие наряду с $\varepsilon$ и другие малые геометрические параметры.

Начнем с одной модельной задачи, содержащей два малых параметра. Именно, рассмотрим периодическую сетку, которая, в отличие от бесконечно тонкой сетки, представленной на рис. 2 , состоит из стержней толшиной $2 h>0$ ( $h$-стержни). Можно представить, что каждый бесконечно тонкий стержень совпадает с продольной осью симметрии соответствуюшего $h$-стержня (см. рис. 7 ).

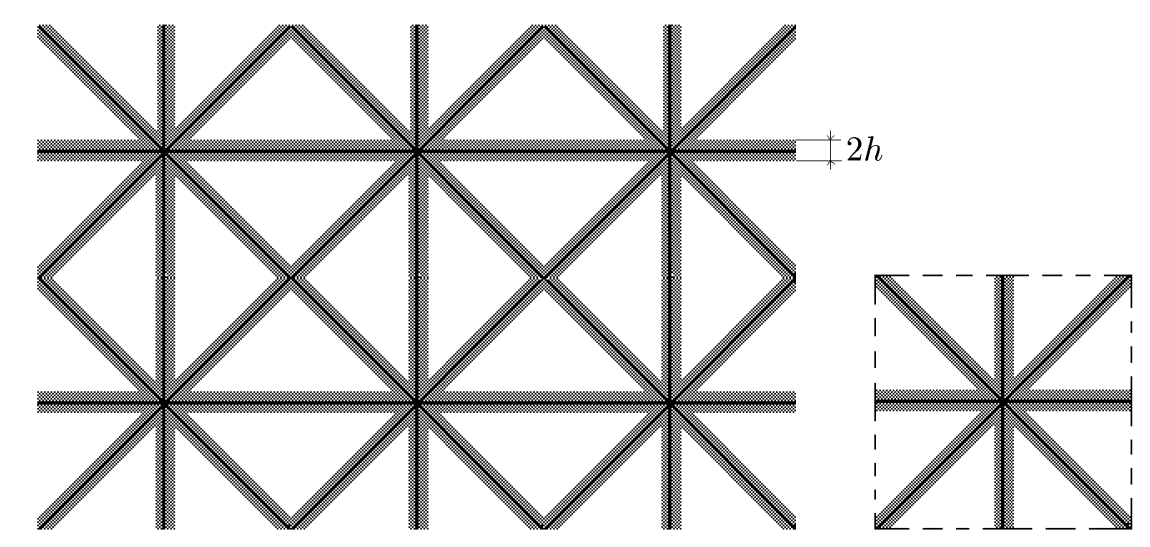

Рис. 7. Тонкая периодическая сетка или стержневая структура 
Введем следуюшие обозначения: $F^{h}$ - тонкая периодическая сетка (рис. 7 ); $\mu^{h}$ периодическая нормированная мера в $\mathbb{R}^{2}$, сосредоточенная на тонкой периодической сетке и пропорциональная на этой сетке плоской мере Лебега; $\mu$ - периодическая нормированная мера, сосредоточенная на бесконечно тонкой периодической сетке (рис. 2) и пропорциональная на этой сетке линейной мере Лебега.

Рассмотрим задачу

$$
u_{\varepsilon, h} \in \mathcal{H}_{0}^{1}\left(\Omega, d \mu_{\varepsilon}^{h}\right), \quad-\operatorname{div} A e\left(u_{\varepsilon, h}\right)+u_{\varepsilon, h}=f,
$$

где $f \in C(\bar{\Omega})^{2}, A$ - постоянный тензор упругости. По определению

$$
\begin{gathered}
u_{\varepsilon, h} \in \mathcal{H}_{0}^{1}\left(\Omega, d \mu_{\varepsilon}^{h}\right), \\
\int_{\Omega}\left[A e\left(u_{\varepsilon, h}\right) \cdot e(\psi)+u_{\varepsilon, h} \cdot \psi\right] d \mu_{\varepsilon}^{h}=\int_{\Omega} f \cdot \psi d \mu_{\varepsilon}^{h}, \quad \psi \in C_{0}^{\infty}(\Omega)^{2} .
\end{gathered}
$$

Выясним, каково поведение $u_{\varepsilon, h}$ при $\varepsilon \rightarrow 0$.

Для фиксированного $h>0$ поведение $u_{\varepsilon, h}$ при $\varepsilon \rightarrow 0$ хорошо известно (теория усреднения в перфорированных областях $\Omega \cap F_{\varepsilon}^{h}$; см. рис. 8).

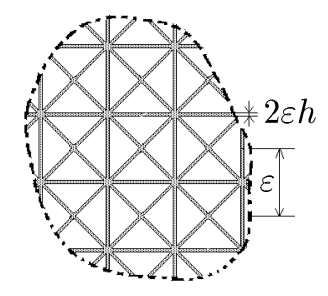

Рис. 8. Перфорированная область $\Omega \cap F_{\varepsilon}^{h}$

Будем считать, что толшина стержней стремится к нулю вместе с периодом $\varepsilon$, T.e.

$$
h=h(\varepsilon) \rightarrow 0 \text { при } \varepsilon \rightarrow 0 .
$$

Такая постановка вопроса не является новой. Например, для скалярных задач в аналогичной ситуации доказана сильная сходимость

$$
\int_{\Omega}\left|u_{\varepsilon, h}(x)-u_{0}(x)\right|^{2} d \mu_{\varepsilon}^{h}=\frac{1}{\left|\Omega \cap F_{\varepsilon}^{h}\right|} \int_{\Omega \cap F_{\varepsilon}^{h}}\left|u_{\varepsilon}(x)-u_{0}(x)\right|^{2} d x \rightarrow 0,
$$

где $u_{0}(x)$ - решение усредненной задачи вида

$$
u_{0} \in H_{0}^{1}(\Omega), \quad-\operatorname{div} A^{\mathrm{hom}} \nabla u_{0}+u_{0}=f .
$$

При этом усредненная матрища определяется по формуле типа (1.7), в которой $\mu-$ мера для бесконечно тонкой сетки. Важно, что предел $u_{\varepsilon, h}$ не зависит от способа стремления параметра $h$ к нулю, (см. $[1$, гл. 8]).

Оказывается, что для теории упругости положение иное: структура усредненной задачи существенно зависит от того, как параметр $h$ стремится к нулю при $\varepsilon \rightarrow 0$ ("масштабный эффект"). Различаются три случая. 
(i) Достаточно толстые стержни: $\lim _{\varepsilon \rightarrow 0} h(\varepsilon) \varepsilon^{-1}=\infty$. Здесь аналогия со скалярными задачами сохраняется. Имеет место сильная сходимость (3.3), где $u_{0}(x)$ - решение классического усредненного уравнения (1.10). Рассмотрим спектральную задачу

$$
u \in H_{0}^{1}(\Omega)^{2}, \quad-\operatorname{div} A^{\mathrm{hom}} e(u)=\lambda u,
$$

или, в краткой форме, $\mathcal{A} u=\lambda u$, где $\mathcal{A}$ - самосопряженный оператор в $L^{2}(\Omega)^{2}$. Для спектра оператора $\mathcal{A}$ имеем

$$
\operatorname{Sp} \mathcal{A}=\left\{\lambda_{1}, \lambda_{2}, \ldots\right\}
$$

где собственные значения $\lambda_{i}$ положительны и конечнократны, $0<\lambda_{1}<\lambda_{2}<\cdots$.

(ii) Достаточно тонкие стержни: $\lim _{\varepsilon \rightarrow 0} h(\varepsilon) \varepsilon^{-1}=0$. В этом случае последовательность $u_{\varepsilon, h}$ не может быть компактной в смысле сходимости (3.3). Поведение решений $u_{\varepsilon, h}$ аналогично поведению решений при $h=0$, т.е. для бесконечно тонкой сетки с мерой $\mu$. Именно, решение $u_{\varepsilon, h}$ "двухмасштабно" сходится к решению неклассического усредненного уравнения для предельной меры $\mu$.

Найдем спектр предельного оператора $\mathcal{A}$, который теперь задан в $L^{2}(\Omega, \mathcal{R})$, а не в $L^{2}(\Omega)^{2}$. Для этого рассмотрим спектральную задачу (2.11). В нашем случае $T=\frac{1}{2} E$, и поэтому

$$
\operatorname{Sp} \mathcal{A}=\left\{0,2 \lambda_{1}, 2 \lambda_{2}, \ldots\right\}
$$

при этом точка $\lambda=0$ является бесконечно кратной.

Итак, при переходе толшины $h$ через критический размер $h \sim \varepsilon$ неосциллируюшее поведение решений меняется на осциллирующее. Кроме того, сушественно меняется спектр предельного оператора, в частности добавляется новая, нулевая, точка спектра.

(iii) Критический случай: $\lim _{\varepsilon \rightarrow 0} h(\varepsilon) \varepsilon^{-1}=\theta>0$ - остается неизученным.

Поясним различие между случаем достаточно толстых и случаем достаточно тонких стержней. Для этого сформулируем один промежуточный результат, справедливый при любом $h(\varepsilon) \rightarrow 0$. Именно, последовательность $u_{\varepsilon, h}$ решений задачи (3.1) (точнее, ее подпоследовательность) имеет "слабый двухмасштабный предел" $u(x, y)$, и для него выполнены свойства (2.5) и (2.6). Эти свойства еще не составляют "усредненное уравнение", так как не определяют однозначно саму вектор-функцию $u(x, y)=u_{0}(x)+\chi(x, y)$. Чтобы получить усредненное уравнение, необходимо найти дополнительную связь между компонентами $u_{0}$ и $\chi$. Это удается сделать отдельно в случае достаточно тонких и в случае достаточно толстых сеток. В первом случае дополнительная связь имеет вид $u-f \perp \mathcal{R}_{1}$ и усредненное уравнение будет неклассическим. Во втором случае дополнительная связь имеет вид $\chi=0$ и (вместе с (2.6)) дает классическое усредненное уравнение.

В критическом случае $h \sim \varepsilon$ связь между компонентами $u_{0}$ и $\chi$ должна быть более сложной и она остается невыясненной. При этом усредненный оператор должен сушественно отличаться от двух ранее найденных - классического и неклассического.

Отметим один любопытный факт, относяшийся к возможной структуре усредненного оператора в критическом случае. 
В обоих некритических случаях для усредненного уравнения выполняется естественное энергетическое равенство. Так, взяв в тождестве (2.10) пробную функцию $\varphi=u$, получим

$$
\int_{\Omega} A^{\text {hom }} e\left(u_{0}\right) \cdot e\left(u_{0}\right) d x+\int_{\Omega} \int_{\square} u \cdot u d x d \mu=\int_{\Omega} \int_{\square} f \cdot u d x d \mu .
$$

Такое же равенство справедливо и для классического усредненного уравнения (1.10) (здесь $u(x, y)=u_{0}(x)$ ). Однако (как показано в $\S 15$ ) в критическом случае для упоминавшейся выше предельной вектор-функции $u(x, y)$ равенство (3.4) не выполняется: левая часть строго меньше правой. Это означает, что в критическом случае левая часть энергетического равенства должна содержать дополнительное слагаемое, которое еще подлежит определению.

Нетрудно сформулировать, в чем состоит “техническое” различие между задачами теории упругости на тонких сетках и аналогичными скалярными задачами.

Справедливо неравенство Пуанкаре

$$
\int_{\square}|u|^{2} d \mu^{h} \leqslant c \int_{\square}|\nabla u|^{2} d \mu^{h}, \quad u \in C_{\mathrm{per}}^{\infty}, \quad \int_{\square} u d \mu^{h}=0,
$$

с постояной $c$, не зависящей от $h$. В силу этого в скалярных задачах толщина $h$ несушественна. Соответствуюшее неравенство Корна выглядит иначе:

$$
\int_{\square} u \cdot u d \mu^{h} \leqslant \frac{c}{h^{2}} \int_{\square} e(u) \cdot e(u) d \mu^{h}, \quad u \in C_{\mathrm{per}}^{\infty}(\square)^{N}, \quad \int_{\square} u d \mu^{h}=0,
$$

причем показатель степени $h$ является точным.

Учитьвая неравенство (3.6), можно заранее сказать, что случай $h \sim \varepsilon$ будет критическим, т.е. будет разделять зоны сушественно различного поведения решений.

3.2. Приведем постановку общей задачи усреднения с двумя малыми параметрами. Ранее мы исходили из периодической меры $\mu$ и вводили исходную задачу (1.3) с помощью скейлинг-меры $\mu_{\varepsilon}$, определенной равенством (1.1). Это была задача с одним малым параметром $\varepsilon$.

Теперь рассмотрим семейство периодических нормированных мер $\mu^{h}, \mu^{h} \rightarrow \mu$. По определению это означает, что

$$
\lim _{h \rightarrow 0} \int_{\square} \varphi d \mu^{h}=\int_{\square} \varphi d \mu, \varphi \in C_{\mathrm{per}}^{\infty}(\square) .
$$

При фиксированном $h>0$ определим скейлинг-меру $\mu_{\varepsilon}^{h}$ по формуле (1.1). После этого будем считать, что $h=h(\varepsilon) \rightarrow 0$. Легко понять, что $d \mu_{\varepsilon}^{h} \rightarrow d x$ при любом $h(\varepsilon) \rightarrow 0$ (см. $\S 11)$.

В качестве исходной задачи рассмотрим задачу (3.1), считая для простоты, что $A$ - постоянный тензор упругости.

Как выбирать меры $\mu^{h}$ ?

Сингулярная периодическая структура задается периодической мерой $\mu$, которая или сингулярна относительно меры Лебега в объемлющем пространстве, как в случае сетки, или содержит сингулярную компоненту, как в случае составной 
структуры. В конкретных примерах всегда имеются "естественные" аппроксимации $\mu^{h}$ такие, что:

(i) $\mu^{h}$ абсолютно непрерывна относительно меры Лебега в объемлющем пространстве;

(ii) $\mu^{h} \rightarrow \mu$.

Например, для периодической сетки меры $\mu^{h}, \mu$, определенные в п. 3.1, этим условиям удовлетворяют.

Рассмотрим пример составной структуры. Поскольку периодичность структуры несущественна, ограничимся рассмотрением плиты $P$ со стержнем $I$ (рис. $1, b)$. Отрезок $I$ заменим полосой $I^{h}$ и зададим меру $\mu^{h}$ так:

$$
d \mu^{h}=\rho^{h} d x, \quad \rho^{h}(x)= \begin{cases}1 & \text { в } P \backslash I^{h}, \\ \frac{1}{\left|I^{h}\right|} & \text { в } I^{h}, \\ 0 & \text { в остальных точках. }\end{cases}
$$

Общим способом аппроксимации служит обычное сглаживание, когда мера $\mu^{h}$ определяется по данной мере $\mu$ равенством

$$
\int_{\square} \varphi d \mu^{h}=\int_{\square}(\varphi)_{h} d \mu, \quad \varphi \in C_{\mathrm{per}}(\square),
$$

где $(\varphi)_{h}$ - сглаживание,

$$
(\varphi)_{h}(x)=h^{-N} \int_{\mathbb{R}^{N}} \varphi(x-y) w\left(h^{-1} y\right) d y
$$

$w$ - достаточно гладкая четная неотрицательная функция, $\int_{\mathbb{R}^{N}} w d y=1$. В этом случае плотность $\rho^{h}(x)$ имеет вид

$$
\rho^{h}(x)=h^{-N} \int_{\mathbb{R}^{N}} w\left(h^{-1}(x-y)\right) d \mu(y) .
$$

K настоящему времени имеется достаточно много работ, в которых изучаются различные асимптотические задачи теории упругости, связанные с аппроксимацией сингулярных структур. Укажем только на работы [9]-[14], где можно найти дальнейшие ссылки.

Приступим к подробному изложению материала настоящей статьи. Нашей целью будет:

(i) исследование свойств соболевских пространств для различных сингулярных объектов;

(ii) доказательство неклассического принщипа усреднения в случае меры $\mu$ обшего вида;

(iii) исследование масштабного эффекта для тонких сеток, а также для некоторых других тонких структур.

В работе широко используется метод двухмасштабной сходимости. Основные свойства этой сходимости приводятся иногда без доказательства. Более подробное изложение этого метода дано в работах [15], [16]. 
Читатель, который хотел бы понять неклассический приншип усреднения без того, чтобы вникать в технику двухмасштабной сходимости, найдет в $§ 18$ вывод этого принщипа с помощью обычного метода асимптотических разложений.

Для чтения настоящей работы не требуется предварительных знаний по усреднению, так как наше изложение более или менее самодостаточно.

Обозначим $\mathbb{R}^{\frac{N(N+1)}{2}}$ множество всех симметрических матриц. Для векторов $a, b \in \mathbb{R}^{N}$ определим матрицу $a \times b=\left\{a_{i} b_{j}\right\}$ (строками служат векторы $b_{j} a$ ). Для $\xi \in \mathbb{R}^{\frac{N(N+1)}{2}}$ имеем

$$
\xi a \cdot b=\xi \cdot(a \times b),
$$

где слева стоит скалярное произведение векторов $\xi a$ и $b$, а справа - скалярное произведение матриц $\xi$ и $a \times b$.

Если функция $\varphi$ и вектор-функция $u$ достаточно гладки, то

$$
e(\varphi u)=\varphi e(u)+\frac{1}{2}[u \times \nabla \varphi+\nabla \varphi \times u] .
$$

Для $\xi \in \mathbb{R}^{\frac{N(N+1)}{2}}$ имеем

$$
e(\varphi u) \cdot \xi=\varphi e(u) \cdot \xi+(u \times \nabla \varphi) \cdot \xi .
$$

Часто используются лебеговы пространства вектор-функций; например,

$$
L^{2}(\Omega)^{\frac{N(N+1)}{2}}=L^{2}\left(\Omega, \mathbb{R}^{\frac{N(N+1)}{2}}\right)
$$

- пространство симметрических квадратично интегрируемых матриц.

Иногда (если это не вызывает недоразумений) мы не различаем векторные и скалярные пространства $L^{2}$.

Если мы хотим подчеркнуть, что $u \in L^{2}(\square, d \mu)$ рассматривается как заданная в $\mathbb{R}^{N}$ периодическая функция, то пишем $u \in L_{\mathrm{per}}^{2}(\square, d \mu)$.

\section{$\S 4$. Скалярные соболевские пространства}

Определим связанные с мерой $\mu$ соболевские пространства $H^{1}(\Omega, d \mu)$ и $H_{\mathrm{per}}^{1}(\square, d \mu)$. В первом случае $\mu-$ конечная борелевская мера в ограниченной области $\Omega$, во втором $\mu$ - периодическая борелевская (нормированная) мера в $\mathbb{R}^{N}$.

Указанные пространства определяются по одному и тому же принципу.

По определению $u \in H^{1}(\Omega, d \mu)$, если

$$
\begin{gathered}
\exists u_{n} \in C^{\infty}(\bar{\Omega}): \quad u_{n} \rightarrow u \text { в } L^{2}(\Omega, d \mu), \\
\nabla u_{n} \rightarrow z \text { в } L^{2}(\Omega, d \mu)^{N} \text { при } n \rightarrow \infty \text { для некоторого } z .
\end{gathered}
$$

Вектор $z$ условимся называть градиентом функиии $u, z=\nabla u$. В общем случае функция $u$ может иметь много градиентов. Очевидно, что множество $\Gamma(u)$ градиентов данной функции $u \in H^{1}(\Omega, d \mu)$ имеет структуру $\Gamma(u)=\nabla u+\Gamma(0)$, где $\nabla u$ - некоторый градиент, а $\Gamma(0)$ - множество градиентов нуля. По определению $g \in \Gamma(0)$, если

$$
\exists \varphi_{n} \in C^{\infty}(\bar{\Omega}): \quad \varphi_{n} \rightarrow 0, \quad \nabla \varphi_{n} \rightarrow g \quad \text { в } \quad L^{2}(\Omega, d \mu) .
$$


Ясно, что множество $Г(0)$ есть замкнутое подпространство векторного пространства $L^{2}(\Omega, d \mu)^{N}$.

Представим некоторый градиент $\nabla u$ в виде суммы двух ортогональных слагаемых:

$$
\nabla u=\nabla^{t} u+g, \quad g \in \Gamma(0), \quad \nabla^{t} u \perp \Gamma(0) .
$$

Очевидно, что первое слагаемое $\nabla^{t} u$ также есть градиент $u$, и этот градиент обладет свойством минимальности:

$$
\int_{\Omega}\left|\nabla^{t} u\right|^{2} d \mu=\min _{\nabla u \in \Gamma(u)} \int_{\Omega}|\nabla u|^{2} d \mu .
$$

Данное определение "минимального" градиента требует знания $\nabla u$ во всей области $\Omega$. Ниже будет показано, что “минимальный” градиент характеризуется некоторыми свойствами поточечной тангенциальности. Оказывается, что само пространство $\Gamma(0)$ допускает поточечное описание, именно найдется $\mu$-измеримое подпространство $T(x) \subset \mathbb{R}^{N}$ такое, что

$$
\Gamma(0)=\left\{g \in L^{2}(\Omega, d \mu)^{N}: g(x) \in T^{\perp}(x)\right\} .
$$

Отсюда следует, что градиент $\nabla u$ можно поточечно проектировать на $T(x)$ и "минимальный" градиент определяется условием тангенциальности $\nabla u(x) \in T(x)$ $\mu$-п.в. Так определенный тангенциальньй градиент, очевидно, поточечно "минимален":

$$
\left|\nabla^{t} u(x)\right| \leqslant|\nabla u(x)| .
$$

Посмотрим, как выглядит множество градиентов нуля на простейших примеpax.

$1^{\circ}$. Отрезок на плоскости. Пусть $I$ - отрезок на оси $O x_{1}$. По определению для $u \in H^{1}(\Omega, d \mu)$

$$
\begin{gathered}
\exists u_{n} \in C^{\infty}(\bar{\Omega}): \quad \int_{I}\left|u-u_{n}\right|^{2} d x_{1} \rightarrow 0 \\
\int_{I}\left|\frac{\partial u_{n}}{\partial x_{1}}-z_{1}\right|^{2} d x_{1} \longrightarrow 0, \quad \int_{I}\left|\frac{\partial u_{n}}{\partial x_{2}}-z_{2}\right|^{2} d x_{1} \rightarrow 0 .
\end{gathered}
$$

Отсюда $u \in H^{1}(I), z_{1}=\frac{\partial u}{\partial x_{1}}$, т.е. $\nabla u=\left\{\frac{\partial u}{\partial x_{1}}, z_{2}\right\}$. Убедимся в том, что компонента $z_{2}$ может быть произвольным элементом из $L^{2}(I)$. Другими словами,

$$
\Gamma(0)=\left\{(0, \alpha): \alpha \in L^{2}(I)\right\}
$$

Поскольку $\Gamma(0)$ замкнуто в $L^{2}(\Omega, d \mu)^{2}$, то достаточно проверить, что $(0, \alpha) \in \Gamma$ для $\alpha \in C_{0}^{\infty}(I)$. В этом случае в определении (4.1) можно взять $\varphi_{n}(x)=x_{2} \alpha\left(x_{1}\right)$.

$2^{\circ}$. Пучок отрезков на плоскости (рис. $1, a$ ). Пусть из начала координат на плоскости "выходят" $k$ отрезков $I_{1}, I_{2}, \ldots, I_{k}$ в направлениях ортов $\lambda^{1}, \lambda^{2}, \ldots, \lambda^{k}$ (см. рис. 9).

Тогда $u \in H^{1}(\Omega, d \mu)$, если: 


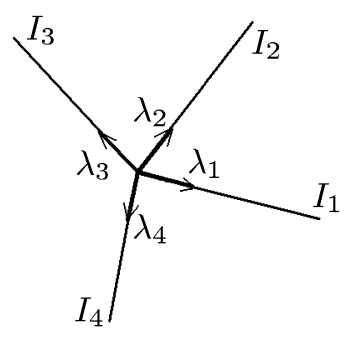

Рис. 9. Пучок отрезков ке $I_{j}$;

(i) $\left.u\right|_{I_{j}} \in H^{1}\left(I_{j}\right)$, где $H^{1}\left(I_{j}\right)$ - одномерное соболевское пространство на отрез-

(ii) значения этих функций $\left.u\right|_{I_{j}}$ совпадают в центре структуры.

Не станем доказывать, что свойства (i) и (ii) являются необходимым и достаточным условием того, что $u \in H^{1}(\Omega, d \mu)$. Для соболевского пространства теории упругости аналогичный факт доказан в $\oint 6$.

Нетрудно выяснить структуру множества $\Gamma(0)$. Если $g \in \Gamma(0)$, то $\left.g \cdot \lambda^{j}\right|_{I_{j}}=0$, как ясно из результатов, полученных для отрезка. Проверим, что $Г(0)=\{g \in$ $\left.L^{2}(\Omega, d \mu)^{2}:\left.g \cdot \lambda^{j}\right|_{I_{j}}=0\right\}$. Для этого рассмотрим $g_{n}(x)=\eta(n x) g(x)$, где

$$
\eta \in C^{\infty}\left(\mathbb{R}^{2}\right), \quad \eta(x)=0 \text { при }|x| \leqslant 1, \quad \chi(x) \equiv 1 \text { при }|x| \geqslant 2 .
$$

Тогда $g_{n} \in \Gamma(0)$ (в силу результатов, полученных для отрезка), $g_{n} \rightarrow g$ в $L^{2}(\Omega, d \mu)$, и поэтому $g \in \Gamma(0)$.

$3^{\circ}$. Составная структура (рис. $1, b$ ). Имеем плиту $P$, где задана плоская мера Лебега, и отрезок $I$ с линейной мерой. Рассмотрим сужение функции $u \in$ $H^{1}(\Omega, d \mu)$ на $P$ и $I$. Очевидно, что $\left.u\right|_{P} \in H^{1}(P),\left.u\right|_{I} \in H^{1}(I)$ и $\left.u\right|_{P \cap I}$ равно следу функции $\left.u\right|_{P}$.

Проверим что, множество $Г(0)$ имеет ту же структуру (4.2), что и в случае отдельного отрезка.

Вектор $g \in \Gamma(0)$ имеет сужения $g_{1}, g_{2} \in L^{2}(P)$ и $\tilde{g}_{1}, \tilde{g}_{2} \in L^{2}(I)$. Из определения градиента нуля ясно, что $g_{1}=g_{2}=\tilde{g}_{1}=0$. Следовательно, $g=(0, \alpha), \alpha \in L^{2}(I)$.

Докажем теперь, что $(0, \alpha) \in \Gamma(0)$, если $\alpha \in L^{2}(I)$. Поскольку Г замкнуто, считаем, что $\alpha$ - гладкая финитная на $I$ функция. Предположим, что отрезок $I$ направлен вдоль оси $O x_{1}$, и рассмотрим

$$
u_{n}(x)=x_{2} \alpha\left(x_{1}\right) \varphi\left(n x_{2}\right)
$$

где $\varphi(t)$ - гладкая финитная функция, $\varphi(0)=1$. Из равенства

$$
\nabla u_{n}=\left\{x_{2} \alpha^{\prime}\left(x_{1}\right) \varphi\left(n x_{2}\right), \alpha\left(x_{1}\right) \varphi\left(n x_{2}\right)+n x_{2} \alpha\left(x_{1}\right) \varphi^{\prime}\left(n x_{2}\right)\right\}
$$

замечаем, что

$$
u_{n} \rightarrow 0, \quad \nabla u_{n} \rightarrow\left\{0, \alpha\left(x_{1}\right)\right\} \quad \text { в } \quad L^{2}(\Omega, d \mu) .
$$


Рассмотрим множество градиентов нуля в пространстве $H_{\mathrm{per}}^{1}(\square, d \mu)$. Для периодической сетки (рис. 2) множество Г (0) - это все векторы из $L^{2}(\square, d \mu)^{2}$, которые на каждом ребре ортогональны этому ребру.

Для периодической составной структуры (рис. 5) Г(0) - это множество векторов, отличных от нуля только на стержнях и ортогональных в каждой точке соответствующему стержню.

Видим, что структура множества градиентов нуля в $H_{\mathrm{per}}^{1}(\square, d \mu)$, по существу, та же, что и в случае $H^{1}(\Omega, d \mu)$. Это обстоятельство не является случайным. Дело в том, что локальная структура $\Gamma(0)$ определяется только мерой $\mu$ и само множество Г $(0)$ может быть описано поточечно. Чтобы понять это, рассмотрим последовательность $\varphi_{n}$ из (4.1), и пусть $a \in C_{0}^{\infty}(\Omega)$. Тогда

$$
a \varphi_{n} \rightarrow 0, \quad \nabla\left(a \varphi_{n}\right)=a \nabla \varphi_{n}+\varphi_{n} \nabla a \rightarrow a g \in \Gamma(0)
$$

Видим, что подпространство Г(0) инвариантно относительно умножения на любую ограниченную функцию:

$$
a \in L^{\infty}(\Omega, d \mu), \quad g \in \Gamma(0) \Longrightarrow a g \in \Gamma,
$$

что влечет за собой важное структурное свойство. Сформулируем это свойство в общем виде.

ЛЕмма 4.1. Пусть Г - подпространство пространства $L^{2}(X, d m)^{s}$, əде $m$ - конечная мера. Если Г инвариантно относительно умножсения на любую функиию $а \in L^{\infty}(X, d m)$, то найдется $т$-измеримое подпространство $T(x) \subset$ $\mathbb{R}^{s}$ такое, что

$$
\Gamma=\left\{g \in L^{2}(X, d \mu)^{s}: g(x) \in T^{\perp}(x) \quad m-n . \text { в. }\right\}
$$

(Эта лемма доказана Дал Масо и автором этой работы; см. [16, § 9].)

Лемма 4.2. Существует $T(x) \subset \mathbb{R}^{N}$ такое, ито множсество градиентов функиии $u \in H_{\mathrm{per}}^{1}$ имеет вид $\nabla u(x)+g(x)$, где $\nabla u(x) \in T(x)$, а $g(x)$ - произвольный вектор из $L^{2}(\square, d \mu)$, $g(x) \subset T^{\perp}$.

Подпространство $T(x)$ называется тангенииальны. мв точке $x$, a $\nabla u(x) \in T(x)$ тангенииальным градиентом. Вообще, вектор $b \in L^{2}(\square, d \mu)^{N}$ называется тангенциальнылм, если $b(x) \in T(x) \mu$-п.в.

\section{§ 5. Соболевские пространства теории упругости}

Начнем с определения пространства $\mathcal{H}_{\text {per }}^{1}(\square, d \mu)$. С этой целью определим $W=$ $W(\square, d \mu)$ как замыкание множества

$$
\left\{u, e(u): u \in C_{\mathrm{per}}^{\infty}(\square)^{N}\right\} \quad \text { в } \quad\left(L^{2}(\square, d \mu)\right)^{N} \times L^{2}(\square, d \mu)^{\frac{N(N+1)}{2}} .
$$

Элементами $W$ служат пары $u, z$, где $u$ - вектор, $z$ - симметрическая матрища. Условимся матрицу z обозначать $e(u)$ и назьвать симметрическим градиентом, 
или просто градиентом и. Совокупность первых компонент назовем соболевским пространством $\mathcal{H}_{\text {per }}^{1}=\mathcal{H}_{\text {per }}^{1}(\square, d \mu)$. Вектор $u \in \mathcal{H}_{\text {per }}^{1}$ может иметь много симметрических градиентов $e(u)$. Множество $\mathcal{E}(u)$ градиентов $u$ имеет структуру $\mathcal{E}(u)=e(u)+\mathcal{E}(0)$, где $\mathcal{E}(0)$ - множество градиентов нуля. По определению

$$
z \in \mathcal{E}(0), \quad \text { если } \exists \varphi_{n} \in C^{\infty}(\square)^{N}: \quad \varphi_{n} \rightarrow 0, \quad e\left(\varphi_{n}\right) \rightarrow z \text { в } L^{2}(\square, d \mu) .
$$

Множество $\mathcal{E}(0)$ обладает теми же свойствами, что и множество $\Gamma(0)$. Так, из (5.1) следует, что $\mathcal{E}(0)$ инвариантно относительно умножения на любую функцию $a \in L_{\text {per. }}^{\infty}$ Исходя из леммы 4.1 получим следуюший результат.

ЛЕмма 5.1. Существует н-измеримое периодическое подпространство $D(x) \subset \mathbb{R}^{\frac{N(N+1)}{2}}$ пространства симметрических матрич такое, что

$$
\mathcal{E}(0)=\left\{z \in L^{2}(\square, d \mu)^{\frac{N(N+1)}{2}}: z(x) \in D^{\perp}(x)\right\} .
$$

Подпространство $D(x)$ также будем называть тангенииальныцм в точке $x$, но использовать его будем только для матрищ (для векторов тангенщиальным остается пространство $T(x))$.

Из этой леммы следует, что всякий вектор $u \in \mathcal{H}_{\mathrm{per}}^{1}$ обладает единственным симметрическим градиентом $e(u)$ таким, что $e(u(x)) \in D(x)$. Такой градиент называется тангенциальным. Симметрическая матрица $z \in L^{2}(\square, d \mu)^{\frac{N(N+1)}{2}}$ называется тангенциальной, если $z(x) \in D(x) \mu$-п.в.

Соотношение между тангенциальными матрицами и векторами дается следующим простым предложением.

ЛЕмма 5.2. Тангенциальная матрица имеет тангенциальные строки.

ДокАЗАТЕЛЬСТво. Для $g \in \Gamma(0)$ имеем

$$
\exists \varphi_{n} \in C_{\mathrm{per}}^{\infty}(\square), \quad \varphi_{n} \rightarrow 0, \quad \nabla\left(\varphi_{n}\right) \rightarrow g \quad \text { в } \quad L^{2}(\square, d \mu) .
$$

Для $u_{n}=\left(\varphi_{n}, 0, \ldots, 0\right)$ выполнено

$$
u_{n} \rightarrow 0, \quad e\left(u_{n}\right) \rightarrow \tilde{g} \in \mathcal{E}(0), \quad \tilde{g}=\left(\begin{array}{cccc}
g_{1} & \frac{1}{2} g_{2} & \ldots & \frac{1}{2} g_{N} \\
\frac{1}{2} g_{2} & 0 & \ldots & 0 \\
\frac{1}{2} g_{3} & 0 & \ldots & 0 \\
\ldots \ldots & \ldots & \ldots & \ldots \\
\frac{1}{2} g_{N} & 0 & \ldots & 0
\end{array}\right) .
$$

Так как тангенциальная матрица $z$ по определению ортогональна $\mathcal{E}(0)$, то

$$
0=\int_{\square} z \cdot \tilde{g} d \mu=\int_{\square}\left(z_{11} g_{1}+z_{12} g_{2}+\cdots+z_{1 N} g_{N}\right) d \mu .
$$

Видим, что первая строка матрицы $z$ ортогональна Г(0). Лемма доказана.

Во всех известных нам примерах наблюдается и обратное: матрица с тангенциальньгми строками сама тангенциальна. Однако в общем случае свойство такого рода не доказано.

Полезно переформулировать лемму 5.2 в более наглядной форме. 
ЛЕмма 5.3. Пусть $b=b(x)$ - тангенииальная матриц $a, g \in \Gamma(0)-$ градиент нуля, $a \in \mathbb{R}^{N}$. Тогда имеет место поточечная ортогональность

$$
b(x) \perp(g(x) \times a) .
$$

Другими словами, $g \in \Gamma(0), \quad a \in \mathbb{R}^{N} \Longrightarrow(g \times a)+(a \times g) \in \mathcal{E}(0)$.

ДокАЗАТЕЛЬСТво. Строки матрищы $b(x)$ тангенщиальны как векторы (по лемме 5.2), а строки матрицы $g \times a$ пропорциональны $g \in \Gamma(0)$. Следовательно, соотношение (5.2) имеет место. Лемма доказана.

Обсудим дальнейшие свойства множества $\mathcal{E}(0)$, рассматривая эллиптические уравнения.

Пусть $A(x)$ - периодический $\mu$-измеримый тензор упругости, подчиненный условию (1.2). Рассмотрим периодическую задачу

$$
u \in \mathcal{H}_{\mathrm{per}}^{1}, \quad-\operatorname{div}(A e(u))+u=f \in L^{2}(\square, d \mu)^{N} .
$$

ОПРЕДЕЛЕНИЕ 5.4. Вектор-функция $u \in \mathcal{H}_{\mathrm{per}}^{1}$ есть решение задачи (5.3), если вьполнено интегральное тождество

$$
\int_{\square}[A e(u) \cdot e(\varphi)+u \cdot \varphi] d \mu=\int_{\square} f \cdot \varphi d \mu, \quad \varphi \in C_{\mathrm{per}}^{\infty}(\square)^{N},
$$

в котором $e(u)$ - некоторый симметрический градиент $u$.

Сушествование и единственность решения в виде пары $u, e(u)$ из $W$ получить совсем легко. Действительно, с помошью левой части тождества (5.4) на пространстве $W$ задается новое скалярное произведение, причем соответствуюшая норма эквивалентна исходной. Поэтому достаточно сослаться на теорему Рисса о представлении линейного функционала в гильбертовом пространстве. Видим, что один вектор из $\mathcal{H}_{\text {per }}^{1}$ и один из его симметрических градиентов удовлетворяют интегральному тождеству. Этот градиент может быть легко найден. Пусть в тождестве (5.4) $\varphi=\varphi_{n}$ - последовательность из (5.1). Тогда получим $A e(u) \perp \mathcal{E}(0)$, т.е. матрица $A e(u)$ тангенциальна.

До сих пор мы говорили о периодических вектор-функциях. Аналогично определяются пространства $\mathcal{H}^{1}=\mathcal{H}^{1}(\Omega, d \mu), \mathcal{H}_{0}^{1}(\Omega, d \mu)$. Для этого в предыдущем определении заменяют множество $C_{\mathrm{per}}^{\infty}(\square)^{N}$ на $C^{\infty}(\bar{\Omega})^{N}$ и $C_{0}^{\infty}(\Omega)^{N}$ соответственно.

Множество $\mathcal{E}(0)$ допускает во всех случаях поточечное описание; для $\mathcal{H}^{1}$ и $\mathcal{H}_{0}^{1}$ оно совпадает с множеством

$$
\left\{z \in L^{2}(\Omega, d \mu)^{\frac{N(N+1)}{2}}: z(x) \in(D(x))^{\perp}\right\} .
$$

Отметим, что ранее мы нигде не говорили о норме в пространствах $\mathcal{H}^{1}$ - норма в них не вводится. В связи с этим отметим следуюший очевидный принцип перехода к пределу в $\mathcal{H}^{1}$.

ПРЕДЛОЖЕНИЕ 5.5. Пусть последовательность $u_{n} \in \mathcal{H}^{1}(\Omega, d \mu)$ такова, что:

(i) $u_{n} \rightarrow u$ в $L^{2}(\Omega, d \mu)$;

(ii) найдутся такие симметрические градиенты е $\left(u_{n}\right)$, которые ограничеHbl в $L^{2}(\Omega, d \mu)^{\frac{N(N+1)}{2}}$.

Тогда $u \in \mathcal{H}^{1}(\Omega, d \mu)$. 


\section{§ 6. Примеры}

Посмотрим на примерах, как устроены пространство $\mathcal{H}^{1}(\Omega, d \mu)$ и множество $\mathcal{E}(0)$. Нас будет интересовать также множество "жестких перемещений"

$$
\mathcal{R}=\left\{u \in \mathcal{H}^{1}(\Omega, d \mu): 0 \in \mathcal{E}(u)\right\} .
$$

$1^{\circ}$. Отрезок на плоскости. Пусть $u=\left(u^{1}, u^{2}\right) \in \mathcal{H}^{1}(\Omega, d \mu)$. По определению обе компоненты $u^{1}, u^{2}$ принадлежат $L^{2}(I)$. Оказывается, что первая компонента обязана принадлежать соболевскому пространству $H^{1}(I)$, а вторая может быть любым элементом из $L^{2}(I)$. Иначе говоря,

$$
\mathcal{H}^{1}=\left\{u=\left(u^{1}, u^{2}\right): u^{1} \in H^{1}(I), \quad u^{2} \in L^{2}(I)\right\},
$$

при этом

$$
e(u)=\left(\begin{array}{cc}
\frac{\partial u^{1}}{\partial x_{1}} & \alpha \\
\alpha & \beta
\end{array}\right), \quad \alpha, \beta \in L^{2}(I) .
$$

Отсюда следует, что множество $\mathcal{E}(0)$ имеет вид

$$
\mathcal{E}(0)=\left\{\left(\begin{array}{cc}
0 & \alpha \\
\alpha & \beta
\end{array}\right), \quad \alpha, \beta \in L^{2}(I)\right\} .
$$

Докажем это. По определению

$$
\begin{gathered}
\exists u_{n} \in C(\bar{\Omega})^{2}: u_{n}^{1} \longrightarrow u^{1}, \quad u_{n}^{2} \longrightarrow u^{2}, \\
e\left(u_{n}\right)=\left(\begin{array}{cc}
\frac{\partial u_{n}^{1}}{\partial x_{1}} & \frac{1}{2} \frac{\partial u_{n}^{1}}{\partial x_{2}}+\frac{1}{2} \frac{\partial u_{n}^{2}}{\partial x_{1}} \\
\frac{1}{2} \frac{\partial u_{n}^{1}}{\partial x_{2}}+\frac{1}{2} \frac{\partial u_{n}^{2}}{\partial x_{1}} & \frac{\partial u_{n}^{2}}{\partial x_{2}}
\end{array}\right) \longrightarrow\left(\begin{array}{cc}
z_{11} & \alpha \\
\alpha & \beta
\end{array}\right)
\end{gathered}
$$

в $L^{2}(I)$. Отсюда ясно, что $u^{1} \in H^{1}(I)$ и $z_{11}=\frac{\partial u^{1}}{\partial x_{1}}$.

Проверим формулу (6.2). Поскольку подпространство $\mathcal{E}(0)$ замкнуто в $L^{2}$, то достаточно убедиться в том, что

$$
\left(\begin{array}{ll}
0 & \alpha \\
\alpha & \beta
\end{array}\right) \in \mathcal{E}(0)
$$

если $\alpha\left(x_{1}\right), \beta\left(x_{1}\right)$ - достаточно гладкие и финитные функции на $I$. В этом случае для вектора

$$
u(x)=\left\{2 x_{2} \alpha\left(x_{1}\right), x_{2} \beta\left(x_{1}\right)\right\}
$$

имеем $u=0, e(u)=\left(\begin{array}{cc}0 & \alpha \\ \alpha & \beta\end{array}\right)$ в $L^{2}(\Omega, d \mu)$, что и требовалось доказать.

Наконец, докажем, что

$$
\mathcal{R}=\left\{\left(c^{1}, u^{2}\right), u^{2} \in L^{2}(I)\right\} .
$$

Достаточно взять $u^{2}=\alpha \in C_{0}^{\infty}(I)$. В этом случае для вектора

$$
u(x)=\left(-x_{2}^{\prime} \alpha^{\prime}\left(x_{1}\right), \alpha\left(x_{1}\right)\right)
$$


имеем $e(u)=0$, и поэтому $(0, \alpha) \in \mathcal{R}$. Обратно, если $e(u)=0$, то из (6.1) видим, что $u^{1}=c^{1}$.

$2^{\circ}$. Пучок стержней на плоскости (рис. 9). Имеет место следующая

ЛЕмма 6.1. Вектор-функиия $и(x)$ из $L^{2}(\Omega, d \mu)^{2}$ принадлежит $\mathcal{H}^{1}(\Omega, d \mu)$ в том и только том случае, когда:

(i) проекции $\left.и \cdot \lambda^{j}\right|_{I_{j}}$ принадлежат одномерным соболевским пространствам $H^{1}\left(I_{j}\right), \quad j=1,2, \ldots, k$;

(ii) найдется постоянный вектор $C$ такой, что

$$
\left.u \cdot \lambda^{j}\right|_{O}=C \cdot \lambda^{j}, \quad j=1,2, \ldots, k,
$$

где $\left.и \cdot \lambda^{j}\right|_{O}$ - предельное значение функции $\left.и \cdot \lambda^{j}\right|_{I_{j}}$ в центре структуры.

Кроме того, для множества $\mathcal{R}$ жестких перемещений имеем

$$
\mathcal{R}: C+v, \quad v \in L^{2}(\Omega, d \mu)^{2},\left.\quad v \cdot \lambda^{j}\right|_{I_{j}}=0
$$

ДокАЗАТЕльСтво. Свойство (i) есть очевидное следствие результатов, полученных для отрезка. Но мы рассмотрим его более подробно, чтобы извлечь дополнительные сведения. По определению

$$
\exists u_{n} \in C^{\infty}(\bar{\Omega}): \quad u_{n} \rightarrow u, \quad e\left(u_{n}\right) \rightarrow e(u) \quad \text { в } \quad L^{2}\left(I_{j}\right), \quad j=1,2, \ldots, k .
$$

Без потери общности считаем $\lambda^{1}=(1,0)$. Тогда $u \cdot \lambda^{1}=u^{1}, e(u) \lambda^{1} \cdot \lambda^{1}=\frac{\partial u^{1}}{\partial x_{1}}$ и из (6.5) следует

$$
u_{n}^{1} \longrightarrow u^{1}, \quad \frac{\partial u_{n}^{1}}{\partial x_{1}} \longrightarrow \frac{\partial u^{1}}{\partial x_{1}}=e(u) \lambda^{1} \cdot \lambda^{1} \quad \text { в } \quad L^{2}\left(I_{1}\right) .
$$

Отсюда $u_{n}^{1} \longrightarrow u^{1}$ равномерно на $I_{1}$, в частности $\left.u_{n}(O) \cdot \lambda^{1} \rightarrow u \cdot \lambda^{1}\right|_{O}$.

Аналогично в общем случае получаем

$$
\left.u_{n}(O) \cdot \lambda^{j} \longrightarrow u \cdot \lambda^{j}\right|_{O}
$$

Если среди векторов $\lambda^{j}$ есть два линейно независимых, то отсюда следует сходимость $u_{n}(O) \longrightarrow C$, и свойство (6.3) доказано. Это свойство выполнено и в случае двух противоположно направленных стержней. Например, если $\lambda^{1}=-\lambda^{2}=$ $(1,0)$, то $u^{1}(x)$ непрерывна в точке $O$ и можно взять $C=\left\{u^{1}(O), c_{2}\right\}$, где $c_{2}$ произвольно.

Теперь докажем, что $u \in \mathcal{H}^{1}$ при выполнении условий (i), (ii). Положим $u_{n}(x)=$ $\eta(n x) u(x)$, где $\eta$ - срезаюшая функция (4.3), и заметим, что вектор $u_{n}$ принадлежит $\mathcal{H}^{1}\left(\Omega, d \mu_{j}\right)$, где $\mu_{j}=\left.\mu\right|_{I_{j}}$. Поскольку $u_{n}$ равен нулю около центра, то $u_{n} \in \mathcal{H}^{1}(\Omega, d \mu)$.

Рассмотрим сначала случай, когда вектор $u$ тангенщиален. Без потери общности считаем, что вектор $C$ из (6.3) равен нулю. Тогда

$$
|u(x)| \leqslant \frac{c}{\sqrt{n}} \text { при }|x| \leqslant \frac{1}{n} .
$$


Зафиксируем некоторые симметрические градиенты $e^{j}(u)$ вектора $u$ как элемента пространства $\mathcal{H}^{1}\left(\Omega, d \mu_{j}\right)$. Тогда из равенства

$$
e\left(u_{n}\right)=\frac{n}{2}(\nabla \eta \times u+u \times \nabla \eta)+\eta \sum e^{j}(u)
$$

и оценки (6.7) легко выводится ограниченность $e\left(u_{n}\right)$ в $L^{2}(\Omega, d \mu)$. Поскольку $u_{n} \rightarrow$ $u$ в $L^{2}(\Omega, d \mu)$, то $u \in \mathcal{H}^{1}(\Omega, d \mu)$ в силу предложения 5.5 .

Пусть теперь вектор $u(x)$ нормален. Тогда $e\left(u_{n}\right)=0$ (равен нулю один из симметрических градиентов, т.е. $\left.0 \in \mathcal{E}\left(u_{n}\right)\right)$. Так как $u_{n} \rightarrow u$ в $L^{2}(\Omega, d \mu)$, то согласно предложению $5.5 u \in \mathcal{H}^{1}(\Omega, d \mu)$. Лемма доказана.

Условие (6.3) выполняется автоматически, если среди ортов $\lambda^{j}$ не более двух линейно независимых. Например, для креста (два пересекающихся стержня) вектор из $\mathcal{H}^{1}(\Omega, d \mu)$ можно задавать независимо на каждом стержне. Если мы имеем три направления, например

$$
\lambda^{1}=(1,0), \quad \lambda^{2}=(0,1), \quad \lambda^{3}=\left(\frac{\sqrt{2}}{2}, \frac{\sqrt{2}}{2}\right),
$$

то сужения $\left.u\right|_{I_{j}}$ уже связаны между собой, а именно

$$
u \in \mathcal{H}^{1}(\Omega, d \mu) \Longleftrightarrow\left\{\begin{array}{c}
u \cdot \lambda^{1} \in H^{1}\left(I_{1}\right), \quad u \cdot \lambda^{2} \in H\left(I_{2}\right), \quad u \cdot \lambda^{3} \in H\left(I_{3}\right), \\
\left.u \cdot \lambda^{3}\right|_{0}=\left.\frac{\sqrt{2}}{2} u \cdot \lambda^{1}\right|_{0}+\left.\frac{\sqrt{2}}{2} u \cdot \lambda^{2}\right|_{0} .
\end{array}\right.
$$

$3^{\circ}$. Периодическая сетка. Рассмотрим сначала простейшие структуры, именно взятый в различных положениях один стержень (см. рис. 10).

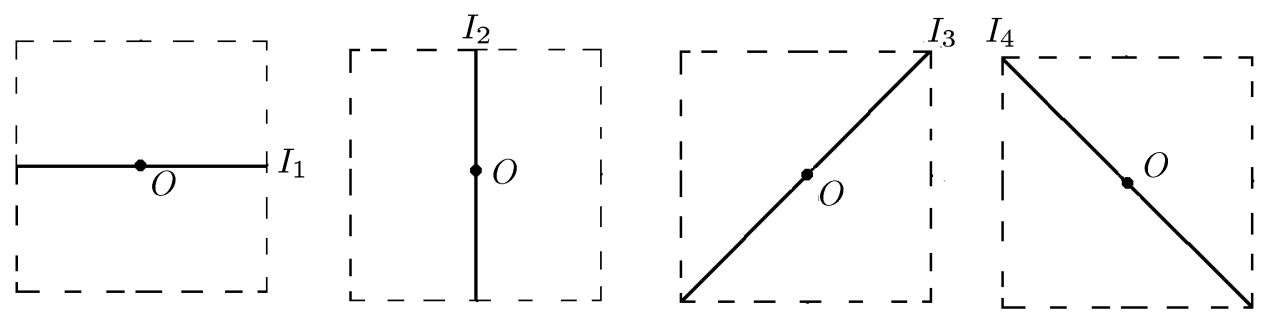

Рис. 10. Периодические системы параллельных стержней

Соответствуюшие пространства обозначим $\mathcal{H}_{\mathrm{per}}^{1}\left(\square, d \mu_{j}\right), j=1,2,3,4$.

В случае $I_{1}$ пространство фактически уже было описано:

$$
\begin{aligned}
\mathcal{H}_{\mathrm{per}}^{1}\left(\square, d \mu_{1}\right): u & =\left(u^{1}, u^{2}\right), \quad u^{1} \in H_{\mathrm{per}}^{1}\left(I_{1}\right), \quad u^{2} \in L^{2}\left(I_{1}\right), \\
e(u) & =\left(\begin{array}{cc}
\frac{\partial u^{1}}{\partial x_{1}} & \beta \\
\beta & \alpha
\end{array}\right), \quad \alpha, \beta \in L^{2}\left(I_{1}\right) .
\end{aligned}
$$

Аналогично оно описывается и для других положений стержня, например

$$
\mathcal{H}_{\mathrm{per}}^{1}\left(\square, d \mu_{4}\right)=\left\{u=\left(u^{1}, u^{2}\right), u^{1}-u^{2} \in H_{\mathrm{per}}^{1}\left(I_{4}\right), u^{1}+u^{2} \in L^{2}\left(I_{4}\right)\right\} .
$$


Условимся нормировать векторы $u=u_{j} \in \mathcal{H}_{\mathrm{per}}^{1}\left(\square, d \mu_{j}\right)$ следуюшим образом: тангенциальная компонента равна нулю в начале координат (и по периодичности во всех точках целочисленной решетки; см. рис. 2), другими словами, $\left.u_{j} \cdot \lambda^{j}\right|_{x=O}=0$, $\lambda^{j}$ - направление стержня. Тогда справедлив "принщип расщепления":

$$
\mathcal{H}_{\mathrm{per}}^{1}(\square, d \mu)=\left\{u=u_{1}+u_{2}+u_{3}+u_{4}+C, \quad u_{j} \in \mathcal{H}_{\mathrm{per}}^{1}\left(\square, d \mu_{j}\right)\right\}
$$

Докажем его. Если $u \in \mathcal{H}_{\text {per }}^{1}(\square, d \mu)$, то в точке $x=O$ выполнено условие согласования (6.3), и поэтому для $u_{j}=\left.(u-C)\right|_{I_{j}}$ имеем $\left.u_{j} \cdot \lambda^{j}\right|_{x=O}=0$.

С другой стороны, достаточно проверить, что для суммы $u_{1}+u_{2}+u_{3}+u_{4}$ выполнено условие согласования в точках пересечения стержней. Для точки $x=O$ условие согласования выполнено в силу нормировки $u_{j}$, а для другой точки условия согласования не требуется, так как в ней пересекаются только два стержня (см. рис. 2).

$4^{\circ}$. Плита со стержнем (рис. $\left.1, b\right)$. Обозначим $I_{1}$ часть стержня $I$, принадлежащую плите $P$, а $I_{2}$ - оставшуюся часть. Компоненты вектора $u \in \mathcal{H}^{1}(\Omega, d \mu)$ принадлежат $L^{2}(P)$ и $L^{2}(I)$. По определению

$$
\begin{aligned}
\exists u_{n} \in C^{\infty}(\bar{\Omega}): & \int_{P}\left(\left|u-u_{n}\right|^{2}+\left|e(u)-e\left(u_{\varepsilon}\right)\right|^{2}\right) d x \longrightarrow 0, \\
& \int_{I}\left(\left|u-u_{n}\right|^{2}+\left|e(u)-e\left(u_{n}\right)\right|^{2}\right) d x_{1} \longrightarrow 0 .
\end{aligned}
$$

Из неравенства Корна

$$
\int_{P}|\nabla v|^{2} d x \leqslant c_{0} \int_{P}\left(|v|^{2}+|e(v)|^{2}\right) d x, \quad v \in C^{\infty}(\bar{P})^{2}
$$

получим

$$
\begin{gathered}
u^{1}, u^{2} \in H^{1}(P), \\
\left.u^{1}\right|_{I_{1}},\left.u^{2}\right|_{I_{1}}-\text { следы функций } u^{1}, u^{2} \text { из } H^{1}(P) \text { на } I_{1}, \\
\left.u^{1}\right|_{I} \in H^{1}(I) .
\end{gathered}
$$

При этом компонента $\left.u^{2}\right|_{I_{2}}$, как увидим ниже, может быть произвольным элементом из $L^{2}\left(I_{2}\right)$. Тензор $e(u)$ имеет вид

$$
e(u)= \begin{cases}\frac{1}{2}\left(\frac{\partial u^{i}}{\partial x_{j}}+\frac{\partial u^{j}}{\partial x_{i}}\right) & \text { на } P, \\
\left(\begin{array}{cc}
\frac{\partial u^{1}}{\partial x_{1}} & \beta \\
\beta & \alpha
\end{array}\right) & \text { на } I .\end{cases}
$$

Проверим, что $\alpha, \beta$ могут быть произвольными функциями из $L^{2}(I)$. Иначе говоря, докажем, что

$$
\mathcal{E}(0)=\left\{z=\left(\begin{array}{ll}
0 & \beta \\
\beta & \alpha
\end{array}\right), \quad \alpha, \beta \in L^{2}(I)\right\} .
$$


Прежде всего, если $z \in \mathcal{E}(0)$, то $z$ имеет вид (6.12), как это ясно из определения $\mathcal{E}(0)$. С другой стороны, поскольку $\mathcal{E}(0)$ замкнуто в $L^{2}$, то можно считать $\alpha, \beta$ гладкими и финитными в $I$. Пусть $\beta=0$, и положим

$$
u_{n}=\left\{0, x_{2} \alpha\left(x_{1}\right) \varphi\left(n x_{2}\right)\right\},
$$

где $\varphi(t)$ - гладкая финитная функция, $\varphi(0)=1$. Тогда из равенства

$$
e\left(u_{n}\right)=\left(\begin{array}{cc}
0 & x_{2} \alpha^{\prime}\left(x_{1}\right) \varphi\left(n x_{2}\right) \\
x_{2} \alpha^{\prime}\left(x_{1}\right) \varphi\left(n x_{2}\right) & \alpha\left(x_{1}\right) \varphi\left(n x_{2}\right)+n x_{2} \alpha\left(x_{1}\right) \varphi^{\prime}\left(n x_{2}\right)
\end{array}\right)
$$

заметим, что

$$
u_{n} \longrightarrow 0, \quad e\left(u_{n}\right) \longrightarrow\left(\begin{array}{cc}
0 & 0 \\
0 & \alpha
\end{array}\right) \quad \text { в } L^{2}(\Omega, d \mu)
$$

Наконец, проверим, что

$$
\mathcal{R}=\left\{C+(0, \alpha): C \in \mathbb{R}^{2}, \quad \alpha \in L^{2}\left(I_{2}\right)\right\} .
$$

Найдем структуру вектора $u \in \mathcal{R}$. Пусть $e(u)=0$. Из (6.10), (6.11) следует, что

$$
\begin{gathered}
u=C+t\left(-x_{2}, x_{1}\right) \text { на } P \text { (жесткое перемещение) }, \\
u^{1}=\text { const на } I .
\end{gathered}
$$

Отсюда $u=C=\left(c_{1}, c_{2}\right)$ на $P$ и $I_{1},\left.u^{1}\right|_{I_{2}}=c_{1}$. Поэтому достаточно взять $\alpha=\left.u^{2}\right|_{I_{2}}-c_{2}$, чтобы получить представление (6.13). С другой стороны, проверим, что $(0, \alpha) \in \mathcal{R}$; можно считать, что $\alpha\left(x_{1}\right)$ - гладкая финитная на $I_{2}$ функция. Но для вектора $u=\left\{0, \alpha\left(x_{1}\right)\right\}$, продолженного нулем на всю структуру, $e(u)=0$ в $L^{2}(\Omega, d \mu)$, и формула $(6.13)$ доказана.

Видим, что трансверсальные жесткие перемешения $\left\{(0, \alpha): \alpha \in L^{2}\left(I_{2}\right)\right\}$ составляют только часть градиентов нуля $\Gamma(0)=\left\{(0, \alpha): \alpha \in L^{2}(I)\right\}$.

$5^{\circ}$. Пластина в трехмерном пространстве (рис. $1, c$ ). Направим ось $O x_{3}$ перпендикулярно пластине $P$. Тогда

$$
\begin{gathered}
\mathcal{H}^{1}: u=\left(u^{1}, u^{2}, u^{3}\right), \quad u^{1}, u^{2} \in H^{1}(P), \quad u^{3} \in L^{2}(P), \\
e(u)=\left(\begin{array}{ccc}
\frac{\partial u^{1}}{\partial x_{1}} & \frac{1}{2} \frac{\partial u^{1}}{\partial x_{2}}+\frac{1}{2} \frac{\partial u^{2}}{\partial x_{1}} & 0 \\
\frac{1}{2} \frac{\partial u^{1}}{\partial x_{2}}+\frac{1}{2} \frac{\partial u^{2}}{\partial x_{1}} & \frac{\partial u^{2}}{\partial x_{2}} & 0 \\
0 & 0 & 0
\end{array}\right)
\end{gathered}
$$

- плоский тензор деформации или тангенциальный симметрический градиент,

$$
\mathcal{E}(0):\left(\begin{array}{ccc}
0 & 0 & \alpha \\
0 & 0 & \beta \\
\alpha & \beta & \gamma
\end{array}\right), \quad \alpha, \beta, \gamma \in L^{2}(P)
$$




\section{$\S 7$. О трансверсальных жестких перемещениях}

Нам потребуются дополнительные сведения о структуре множества $\mathcal{R}$ периодических жестких перемешений.

Из определения (1.6) имеем $\mathcal{R}=\left\{u \in \mathcal{H}_{\text {per }}^{1}(\square, d \mu): 0 \in \mathcal{E}(u)\right\}$. Очевидно, что условие $0 \in \mathcal{E}(u)$ эквивалентно условию $\mathcal{E}(u)=\mathcal{E}(0)$.

Множество $\mathcal{R}$ не является инвариантным относительно умножения на гладкую периодическую функцию $\varphi$ (например, $c \in \mathcal{R}$, но $\varphi c \bar{\in} \mathcal{R}$ ). Покажем, что инвариантностью обладает его часть, состоящая из трансверсальных перемещений, именно $\mathcal{R}_{1}=\mathcal{R} \cap \Gamma(0)$, где $\Gamma(0)$ - множество градиентов нуля в $H_{\mathrm{per}}^{1}(\square, d \mu)$.

ЛЕмма 7.1. Имеем

$$
g \in \mathcal{R} \cap \Gamma(0), \quad \varphi \in C_{\text {per }}^{\infty} \Longrightarrow \varphi g \in \mathcal{R} \cap \Gamma(0) .
$$

ДокАЗАТЕЛЬСТво. Проверим, что $0 \in \mathcal{E}(\varphi g)$, так как условие $\varphi g \in \Gamma(0)$ выполнено в силу инвариантности $Г(0)$. Исходим из обычной формулы

$$
e(\varphi g)=\varphi e(g)+\frac{1}{2}[g \times \nabla \varphi+\nabla \varphi \times g] .
$$

Ясно, что получим элемент из $\mathcal{E}(\varphi g)$, если в качестве $e(g)$ в этой формуле возьмем любой элемент из $\mathcal{E}(g)=\mathcal{E}(0)$, в частности матрицу

$$
\frac{1}{2}[g \times a+a \times g], \quad a \in \mathbb{R}^{N},
$$

принадлежащую $\mathcal{E}(0)$ по лемме 5.3. Поскольку $\mathcal{E}(0)$ описывается поточечно, то можно взять $a=a(x) \in L^{\infty}(\square, d \mu)^{N}$. Итак, множество $\mathcal{E}(\varphi g)$ содержит матрищы вида

$b(x)=\frac{1}{2} \varphi(x)[g(x) \times a(x)+a(x) \times g(x)]+\frac{1}{2}[g(x) \times \nabla \varphi(x)+\nabla \varphi(x) \times g(x)], \quad a \in\left(L^{\infty}\right)^{N}$.

Допустим сначала, что $\varphi>0$. Тогда, взяв $a=-\frac{\nabla \varphi}{\varphi}$, получим $b \equiv 0$. Видим, что условие $\varphi g \in \mathcal{R}$ выполнено для положительных $\varphi$, но тогда оно, очевидно, выполнено для любых $\varphi$. Чтобы убедиться в этом, достаточно рассмотреть функцию $\varphi$ как разность положительных функций и воспользоваться линейностью множества $\mathcal{R}$. Лемма доказана.

Необходимо отметить, что $\mathcal{R}_{1} \neq \Gamma(0)$, как это мы видели на примере составной структуры (см. (6.13)). Во всех примерах (за исключением системы из одного стержня) справедливо разложение $\mathcal{R}=\mathbb{R}^{N}+\mathcal{R}_{1}$, т.е. всякое жесткое перемещение сводится к постоянному и трансверсальному. Однако в общем виде такое свойство не доказано.

В пространстве $\mathcal{H}_{0}^{1}(\Omega, d \mu)$ так же можно выделить множество трансверсальных жестких перемешений и доказать для него свойство инвариантности. В полном объеме эти результаты нам не понадобятся.

Для дальнейшего важна только определенная связь между жесткими перемещениями в $\mathcal{H}_{\mathrm{per}}^{1}(\square, d \mu)$ и жесткими перемещениями в $\mathcal{H}_{0}^{1}\left(\Omega, d \mu_{\varepsilon}\right)$.

Рассмотрим в области $\Omega$ вектор-функцию

$$
\begin{gathered}
\psi(x)=\varphi(x) g(y), \quad y=\varepsilon^{-1} x, \quad \varphi \in C_{0}^{\infty}(\Omega), \\
g \in \mathcal{H}_{\mathrm{per}}^{1}(\square, d \mu), \quad g \in \mathcal{R} \cap \Gamma(0)=\mathcal{R}_{1} .
\end{gathered}
$$


ЛЕмма 7.2. Вектор-функция $\psi$, представленная в (7.1), принадлежит пространству $\mathcal{H}_{0}^{1}\left(\Omega, d \mu_{\varepsilon}\right)$ и является в нем жестким перемещением. Это остается в силе, если в (7.1) взять $\varphi \in C(\bar{\Omega})$ или $\varphi \in L^{\infty}(\Omega)$.

ДокАЗАТЕЛЬСТво. Можно считать, что $\varepsilon=1$, а носитель функции $\varphi \in C_{0}^{\infty}(\Omega)$ содержится внутри ячейки периодичности $\square$. Продолжим $\varphi$ периодически на $\mathbb{R}^{N}$. По лемме 7.1

$$
\exists u_{n} \in C_{\text {per }}^{\infty}(\square)^{N}: u_{n} \longrightarrow \varphi g, \quad e\left(u_{n}\right) \longrightarrow 0 \quad \text { в } \quad L^{2}(\square, d \mu) .
$$

Рассмотрим срезающую функцию $\eta \in C_{0}^{\infty}(\Omega), \eta \equiv 1$ в окрестности $\operatorname{supp} \varphi$. Тогда для $v_{n}=\eta u_{n}$ имеем

$$
\begin{gathered}
v_{n} \in C_{0}^{\infty}(\Omega), \quad v_{n} \rightarrow \varphi g, \\
e\left(v_{n}\right)=\eta e\left(u_{n}\right)+\frac{1}{2}\left(u_{n} \times \nabla \eta+\nabla \eta \times u_{n}\right) \rightarrow \frac{1}{2}(\varphi g \times \nabla \eta+\nabla \eta \times \varphi g)=0
\end{gathered}
$$

в $L^{2}(\Omega, d \mu)$. Тем самым проверено, что $\varphi g$ есть жесткое перемешение в $\mathcal{H}_{0}^{1}(\Omega, d \mu)$.

\section{§ 8. Усредненный тензор}

Будем рассматривать симметрические матрицы и векторы из $L^{2}(\square, d \mu)$.

Определим потенциальные и соленоидальные матрицы на торе периодичности. Введем пространство $V_{\text {pot }}$ потенциальны $x$ матриц как замыкание множества $\left\{e(\varphi): \varphi \in C_{\mathrm{per}}^{\infty}(\square)^{N}\right\}$ в $L^{2}(\square, d \mu)^{\frac{N(N+1)}{2}}$.

По определению матрища $b$ соленоидальн $a, \operatorname{div} b=0$ (в смысле меры $\mu$ ), если

$$
\int_{\square} b \cdot e(\varphi) d \mu=0 \quad \forall \varphi \in C_{\mathrm{per}}^{\infty}(\square)^{N} .
$$

Таким образом, имеем ортогональное разложение

$$
L^{2}(\square, d \mu)^{\frac{N(N+1)}{2}}=\mathcal{V}_{\text {pot }} \oplus \mathcal{V}_{\text {sol }}
$$

Далее, пусть $b$ - матрица и $a-$ вектор; тогда $\operatorname{div} b=a($ в смысле меры $\mu)$, если

$$
\int_{\square} b \cdot e(\varphi) d \mu=-\int_{\square} a \cdot \varphi d \mu, \varphi \in C_{\mathrm{per}}^{\infty}(\square)^{N} .
$$

В этом случае также используются выражения: "матрица $b$ имеет дивергенцию из $L^{2}$ ", "вектор $a$ допускает представление в виде дивергенции".

Из этих определений ясно, что соленоидальные матрищы тангенциальны.

Тангенщиальными будут также матрищы с дивергенцией из $L^{2}$. Для доказательства этого достаточно в тождестве (8.2) взять $\varphi=\varphi_{n}$ - последовательность из (5.1).

Может случиться, что ненулевая постоянная матрица является потенциальной. Обозначим через $L$ подпространство в $\mathbb{R}^{\frac{N(N+1)}{2}}$, состоящее из потенциальных 
матриц. Мера $\mu$ называется невырожденной, если $L=\{0\}$. Невырожденность меры $\mu$ тесно связана с невырожденностью усредненного тензора $A^{\text {hom }}$ (см. (1.7)),

$$
A^{\mathrm{hom}} \xi \cdot \xi=\min _{v \in \mathcal{V}_{\text {pot }}}\langle A(\xi+v) \cdot(\xi+v)\rangle
$$

Из этой формулы видно, что тензор $A^{\text {hom }}$ (как неотрицательный самосопряженньй оператор в пространстве симметрических матриш) имеет своим ядром подпространство $L$, а областью значений - ортогональное дополнение $\tilde{L}=L^{\perp}$. Уравнение Эйлера задачи (8.3) имеет вид

$$
v \in \mathcal{V}_{\text {pot }}, \quad\langle A(\xi+v) \cdot \varphi\rangle=0 \quad \forall \varphi \in \mathcal{V}_{\text {pot }} \quad \text { или } \operatorname{div}(A(\xi+v))=0 .
$$

Поэтому $A^{\text {hom }} \xi \cdot \xi=\langle A(\xi+v) \cdot(\xi+v)\rangle=\langle A(\xi+v)\rangle \cdot \xi$,

$$
A^{\text {hom }} \xi=\langle A(\xi+v)\rangle, \quad A(\xi+v) \in \mathcal{V}_{\text {sol }} .
$$

Каждый элемент из $\tilde{L}$ является средним значением некоторой соленоидальной матрицы (см. (8.5)). Верно и обратное: среднее значение любой соленоидальной матрицы принадлежит $\tilde{L}$. Действительно, если $b \in \mathcal{V}_{\text {sol }}$, то $0=\langle b \cdot \xi\rangle=\langle b\rangle \cdot \xi$ для $\xi \in L$.

Итак, справедливо следующее

ПРЕДЛОЖЕНИЕ 8.1. Пусть $L$ - подпространство из $\mathbb{R}^{\frac{N(N+1)}{2}}$, состоящее из потенциальных матриц, $\tilde{L}$ - его ортогнальное дополнение. Тогда усредненный тензор является нулевым на L и положстельно определенным на $\tilde{L}$. Пространство $\tilde{L}$ - это в точности совокупность средних значений соленоидальных матриц.

Вычислим усредненный тензор для сетки, считая, что исходный тензор $A$ постоянен и изотропен (см. (1.11)). Начнем с рассмотрения простейшей структуры, состоящей из параллельных горизонтальных стержней (рис. 4). В этом случае (см. $(6.8))$

$$
V_{\mathrm{pot}}=\left\{\left(\begin{array}{cc}
\frac{\partial u^{1}}{\partial x_{1}} & \beta \\
\beta & \alpha
\end{array}\right): u^{1} \in H_{\mathrm{per}}^{1}\left(I_{1}\right), \quad \alpha, \beta \in L^{2}\left(I_{1}\right)\right\},
$$

и требуется найти

$$
\begin{aligned}
& \min _{\substack{u^{1} \in H_{\text {per }}^{1}\left(I_{1}\right) \\
\alpha, \beta \in L^{2}\left(I_{1}\right)}} \int_{0}^{1}\left[k\left(\xi_{11}+\frac{\partial u^{1}}{\partial x_{1}}\right)^{2}+2 k\left(\xi_{12}+\beta\right)^{2}+k\left(\xi_{22}+\alpha\right)^{2}\right. \\
& \left.\quad+k_{1}\left(\xi_{11}+\frac{\partial u^{1}}{\partial x_{1}}+\xi_{22}+\alpha\right)^{2}\right] d x_{1} .
\end{aligned}
$$

Несложными вычислениями можно получить величину $\hat{k} \xi_{11}^{2}$, где $\hat{k}=k_{1} \frac{k+2 k_{1}}{k+k_{1}}$, а для других положений стержня - величину $\hat{k}(\xi \lambda \cdot \lambda)^{2}$, где $\lambda=(0,1),\left(\frac{\sqrt{2}}{2}, \pm \frac{\sqrt{2}}{2}\right)$.

Далее, согласно принципу расщепления (6.9) форма $A^{\text {hom }} \xi \cdot \xi$ будет равна сумме найденных элементарных форм, взятых с весами $\rho_{1}, \rho_{2}, \rho_{3}, \rho_{4}$. Приходим к формуле (1.12). 
Для периодических сеток пространство $\mathcal{V}_{\text {sol }}$ имеет весьма простую структуру. Действительно, из (6.9) следует, что

$$
\operatorname{div} b=0 \text { по мере } \mu \Longleftrightarrow \operatorname{div} b=0 \text { по мерам } \mu_{1}, \mu_{2}, \mu_{3}, \mu_{4} .
$$

Пусть мера $\mu_{1}$ отвечает системе горизонтальных стержней. Матрица $b$ тангенциальна, т.е. имеет вид $b=c\left(\begin{array}{ll}1 & 0 \\ 0 & 0\end{array}\right)$, причем $c=$ const, поскольку

$$
0=\int_{\square} b \cdot e(\varphi) d \mu_{1}=\int_{I_{1}} c \frac{\partial \varphi_{1}}{\partial x_{1}} d x_{1} \quad \forall \varphi_{1} \in C_{\mathrm{per}}^{\infty}(\square) .
$$

Для стержня, имеюшего направление $\lambda$, соленоидальная матрица пропорциональна матрице $\lambda \times \lambda$. Итак, в случае сеток пространство $\mathcal{V}_{\text {sol }}-$ это совокупность периодических матриц, которые постоянны и тангенциальны на каждом стержне.

Укажем другой способ вычисления тензора $A^{\text {hom }}$ для сетки, основанный на точном решении уравнения Эйлера (8.4). Снова рассмотрим случай горизонтальных стержней. Пусть $v$ - решение уравнения (8.4). Тогда матрица $A(\xi+v)$ соленоидальна,

$$
A(\xi+v)=\left(\begin{array}{ll}
c & 0 \\
0 & 0
\end{array}\right),
$$

где $c$ - константа, которую требуется найти.

Для изотропного тензора $\mathcal{A}$ (см. (1.11)) легко вычислить обратный тензор:

$$
A^{-1} \eta=\frac{\eta}{k}-\frac{k_{1}}{k\left(k+2 k_{1}\right)} E \operatorname{tr} \eta
$$

Поэтому

$$
\xi+v=A^{-1}\left(\begin{array}{cc}
c & 0 \\
0 & 0
\end{array}\right)=\frac{1}{k}\left(\begin{array}{cc}
c & 0 \\
0 & 0
\end{array}\right)-\frac{c k_{1}}{k\left(k+2 k_{1}\right)}\left(\begin{array}{ll}
1 & 0 \\
0 & 1
\end{array}\right) .
$$

Отсюда видно, что решение $v=\left(\begin{array}{ll}v_{11} & v_{12} \\ v_{12} & v_{22}\end{array}\right)$-также постоянная матрица. Так как она по определению потенциальна, то $v_{11}=0$. Это позволяет из (8.6) найти константу $c$, именно

$$
c=\frac{k\left(k+2 k_{1}\right)}{k+k_{1}} \xi_{11}=\hat{k} \xi_{11} .
$$

Отсюда $A^{\text {hom }} \xi \cdot \xi=\langle A(\xi+v) \cdot \xi\rangle=\hat{k} \xi_{11}^{2}$.

Чтобы решить уравнение Эйлера для сетки, нужно просто объединить решения для отдельных стержней. Любопытно, что решение является симметрическим градиентом нуля, т.е. $v \in \mathcal{E}(0)$.

Имеется естественная связь между потенциальными матрицами и элементами соболевского пространства $\mathcal{H}_{\mathrm{per}}^{1}$. Так, симметрический градиент любой векторфункции из $\mathcal{H}_{\text {per }}^{1}$ принадлежит $\mathcal{V}_{\text {pot. }}$. Обратное утверждение справедливо лишь при некоторых дополнительных условиях; в частности, таким условием служит неравенство Корна

$$
\int_{\square} \varphi^{2} d \mu \leqslant c \int_{\square} e(\varphi) \cdot e(\varphi) d \mu, \quad \varphi \in \mathcal{H}_{\mathrm{per}}^{1}(\square, d \mu), \quad \varphi \perp \mathcal{R},
$$

где $e(\varphi)$ - некоторьй симметрический градиент $\varphi$, например тангенциальный. 
Из этого неравенства нетрудно вывести, что всякая потенщиальная матрища $v \in$ $\mathcal{V}_{\text {pot }}$ допускает единственное представление в виде

$$
v=e(w), \quad w \in \mathcal{H}_{\mathrm{per}}^{1}, \quad w \perp \mathcal{R}
$$

Неравенство (8.7) элементарно проверяется для конкретных примеров (сетки, составная структура, ящичная структура). Для доказательства неклассического принщипа усреднения оно не требуется.

\section{§ 9. Основные результаты о двухмасштабной сходимости}

9.1. Напомним определение и основные свойства двухмасштабной сходимости.

Пусть $\mu$ - периодическая нормированная борелевская мера, $\mu_{\varepsilon}-$ скейлинг-мера, определенная равенством (1.1). Напомним также свойство среднего значения периодической функиии: для $\varphi \in C_{0}^{\infty}(\Omega), b \in C_{\mathrm{per}}^{\infty}(\square)$

$$
\lim _{\varepsilon \rightarrow 0} \int_{\Omega} \varphi(x) b\left(\varepsilon^{-1} x\right) d \mu_{\varepsilon}=\int_{\Omega} \int_{\square} \varphi(x) b(y) d x d \mu
$$

(доказательство см. в $\S 11$, где разобран более общий случай).

Рассмотрим последовательность функций $v_{\varepsilon}(x) \in L^{2}\left(\Omega, d \mu_{\varepsilon}\right)$, всегда предполагая ее ограниченной (см. (1.5)), и пробные функции

$$
\Phi(x, y)=\varphi(x) b(y), \quad \varphi \in C_{0}^{\infty}(\Omega), \quad b \in C_{\mathrm{per}}^{\infty}(\square)
$$

ОПРЕДЕЛЕНИЕ 9.1. Последовательность $v_{\varepsilon}(x)$ cлабо двухмаситабно сходится $к$ функиии $v=v(x, y) \in L^{2}(\Omega \times \square, d x \times d \mu)=L^{2}(\Omega \times \square), v_{\varepsilon}(x) \stackrel{2}{\rightarrow} v(x, y)$, если

$$
\lim _{\varepsilon \rightarrow 0} \int_{\Omega} \Phi\left(x, \varepsilon^{-1} x\right) v_{\varepsilon}(x) d \mu_{\varepsilon}=\int_{\Omega} \int_{\square} \Phi(x, y) v(x, y) d x d \mu
$$

для любой пробной функции $\Phi$ из (9.1).

Рассмотрим простейший пример: если $v_{\varepsilon}(x)=\Phi\left(x, \varepsilon^{-1} x\right)$, то $v_{\varepsilon}(x) \stackrel{2}{\rightarrow} \Phi(x, y)$. Это следует из свойства среднего значения.

Перечислим основные свойства слабой двухмасштабной сходимости. Начнем с принципа выбора.

(i) Ограниченная последовательность $v_{\varepsilon}$ компактна в смысле слабой двухмаситабной сходимости.

(Доказательство см. в $\S 11$, где рассмотрен более общий случай.)

В определении 9.1 использован минимально узкий класс пробных функщий. Его можно по-разному расширять с сохранением сходимости (9.2).

(ii) Сходимость (9.2) сохраняется для пробных функиий $\Phi(x, y)=\varphi(x) b(y)$, әде $\varphi(x) \in C(\bar{\Omega})$ u $b(y) \in L^{2}(\square, d \mu)$.

(Доказательство см. в $\S 11$, где рассмотрен более общий случай.)

Сравним обычный слабый предел с двухмасштабным.

(iii) $E c л и v_{\varepsilon}(x) \stackrel{2}{\rightarrow} v(x, y)$, mо $v_{\varepsilon}(x) \rightarrow \int_{\square} v(x, y) d \mu(y)$, m.е. обычный предел получим из двухмасштабного усреднением по периоду.

(Для доказательства нужно взять в (9.2) пробную функцию $\Phi=\varphi(x)$ ). 
(iv) $E c л u v_{\varepsilon}(x) \stackrel{2}{-} v(x, y), \quad a \in L_{\text {per }}^{\infty}(\square, d \mu)$, mo $a\left(\varepsilon^{-1} x\right) v_{\varepsilon}(x) \stackrel{2}{\rightarrow} a(y) v(x, y)$. Это свойство непосредственно следует из (ii).

(v) Пусть функиия $f(y, \xi)$ измерима и периодична по $y \in \mathbb{R}^{N}$, выпукла по $\xi \in \mathbb{R}^{s}$ и подчинена квадратичной оченке

$$
0 \leqslant f(y, \xi) \leqslant c\left(\xi^{2}+1\right) .
$$

Если для вектора $v_{\varepsilon}(x) \in L^{2}\left(\Omega, d \mu_{\varepsilon}\right)^{s}$ имеет место сходимость $v_{\varepsilon}(x) \stackrel{2}{\rightarrow}$ $v(x, y), m o$

$$
\liminf _{\varepsilon \rightarrow 0} \int_{\Omega} f\left(\varepsilon^{-1} x, v_{\varepsilon}(x)\right) d \mu_{\varepsilon} \geqslant \int_{\Omega} \int_{\square} f(y, v(x, y)) d x d \mu
$$

в частности

$$
\liminf _{\varepsilon \rightarrow 0} \int_{\Omega}\left|v_{\varepsilon}(x)\right|^{2} d \mu_{\varepsilon} \geqslant \int_{\Omega} \int_{\square}|v(x, y)|^{2} d x d \mu
$$

ОПРЕДЕЛЕНИЕ 9.2. Последовательность $v_{\varepsilon}$ сильно двухмасштабно сходится $\kappa v(x, y)$, если

$$
\lim _{\varepsilon \rightarrow 0} \int_{\Omega} v_{\varepsilon}(x) z_{\varepsilon}(x) d \mu_{\varepsilon}=\int_{\Omega} \int_{\square} v(x, y) z(x, y) d x d \mu, \quad \text { как только } z_{\varepsilon}(x) \stackrel{2}{\rightarrow} z(x, y) .
$$

Рассмотрим простой пример: если $v_{\varepsilon}(x)=\varphi(x) b\left(\varepsilon^{-1} x\right), \quad \varphi \in C(\bar{\Omega}), \quad b \in$ $L_{\text {per }}^{2}(\square, d \mu)$, то $v_{\varepsilon}(x) \stackrel{2}{\longrightarrow} \varphi(x) b(y)$. Это следует из свойства (ii) слабой двухмасштабной сходимости.

Отметим следуюшие свойства сильной двухмасштабной сходимости.

(i) Выполнено $v_{\varepsilon}(x) \stackrel{2}{\longrightarrow} v(x, y)$ тогда и только тогда, когда $v_{\varepsilon} \stackrel{2}{\rightarrow} v u$

$$
\lim _{\varepsilon \rightarrow 0} \int_{\Omega}\left|v_{\varepsilon}\right|^{2} d \mu_{\varepsilon}=\int_{\Omega} \int_{\square}|v|^{2} d x d \mu .
$$

(ii) Если предельная функция $v(x, y)$ достаточно гладкая, например имеет вид

$$
v(x, y)=\sum \varphi_{i}(x) b_{i}(y)
$$

әде $\varphi_{i} \in C(\bar{\Omega}), \quad b_{i} \in L_{\mathrm{per}}^{2}(\square, d \mu), m o$

$$
v_{\varepsilon} \stackrel{2}{\longrightarrow} v \Leftrightarrow \lim _{\varepsilon \rightarrow 0} \int_{\Omega}\left|v_{\varepsilon}(x)-v\left(x, \varepsilon^{-1} x\right)\right|^{2} d \mu_{\varepsilon}=0 .
$$

Доказательство этих свойств можно найти в работах [15], [16]. Кроме того, в $\S 11$ развивается более общий подход к теории двухмасштабной сходимости и некоторые свойства доказаны заново. 
9.2. Выясним наиболее интересные свойства двухмасштабной сходимости, которые обнаруживаются при совместном рассмотрении вектор-функции $u_{\varepsilon}(x)$ и ее тензора деформации $e\left(u_{\varepsilon}\right)$. Начнем с важной леммы об аппроксимации.

Пусть вектор $a \in L^{2}(\square, d \mu)^{N}$ допускает представление в виде дивергенции, $a=\operatorname{div} b$, т.е. имеет место тождество (8.2) для некоторой симметрической матришы $b \in L^{2}(\square, d \mu)^{\frac{N(N+1)}{2}}$. Взяв в этом тождестве $\varphi=\varphi_{n}$, где $\varphi_{n}$ - последовательность из (1.6), получим $a \perp \mathcal{R}$. Это - необходимое условие представления вектора в виде дивергенции.

ЛЕмма 9.3 (об аппроксимации). Множество $S$ векторов $а$, допускающих представление $a=\operatorname{div} b$, плотно в $\mathcal{R}^{\perp}$.

ДОКАЗАТЕЛЬСТво. Пусть

$$
f \perp \mathcal{R}, \quad f \perp S .
$$

Рассмотрим периодическую задачу $-\operatorname{div} e(u)+u=f$. Из интегрального тождества

$$
\int_{\square}[e(u) \cdot e(\varphi)+u \cdot \varphi] d \mu=\int_{\square} f \cdot \varphi d \mu
$$

имеем

$$
\begin{gathered}
\int_{\square} e(u) \cdot e(u) d \mu+\int_{\square} u \cdot u d \mu=\int_{\square} u \cdot f d \mu, \\
\int_{\square} u \cdot u d \mu \leqslant \int_{\square} f \cdot f d \mu .
\end{gathered}
$$

Из (9.8) видим, что $f-u \in S$, и поэтому $f-u \perp f$, т.е.

$$
\int_{\square} f \cdot f d \mu=\int_{\square} u \cdot f d \mu \leqslant \int_{\square} u \cdot u d \mu .
$$

Отсюда и из $(9.9),(9.7)$ последовательно получаем

$$
\int_{\square} f \cdot f d \mu=\int_{\square} u \cdot u d \mu \Longrightarrow e(u)=0 \Longrightarrow u \in \mathcal{R}, \quad u=f \in \mathcal{R} \Longrightarrow f=0 .
$$

Лемма доказана.

Используем лемму об аппроксимации для доказательства следующей теоремы.

ТЕОРема 9.4. Пусть $u_{\varepsilon} \in C^{\infty}(\bar{\Omega})^{N}$ и выполнены условия

(i) $u_{\varepsilon}(x) \stackrel{2}{\rightarrow} u(x, y)$;

(ii) $\varepsilon e\left(u_{\varepsilon}\right) \longrightarrow 0$ в $L^{2}\left(\Omega, d \mu_{\varepsilon}\right)$.

Тогда $u(x, y) \in L^{2}(\Omega, \mathcal{R})$.

ДоказАТельство. Возьмем $a=\operatorname{div} b$. Сначала заметим, что тождество (8.2) на периодических функциях влечет за собой тождество на финитных функциях:

$$
\varepsilon \int_{\Omega} b\left(\varepsilon^{-1} x\right) \cdot e(\psi(x)) d \mu_{\varepsilon}=-\int_{\Omega} a\left(\varepsilon^{-1} x\right) \cdot \psi(x) d \mu_{\varepsilon}, \quad \psi \in C_{0}^{\infty}(\Omega)^{N} .
$$


Полагая здесь $\psi(x)=\varphi(x) u_{\varepsilon}(x)$, где $\varphi \in C_{0}^{\infty}(\Omega)$, и используя $(3.10),(3.11)$, получим

$$
\begin{aligned}
-\varepsilon \int_{\Omega} e\left(u_{\varepsilon}(x)\right) \cdot \varphi b\left(\varepsilon^{-1} x\right) d \mu_{\varepsilon}= & \int_{\Omega} \varphi(x) a\left(\varepsilon^{-1} x\right) \cdot u_{\varepsilon}(x) d \mu_{\varepsilon} \\
& +\varepsilon \int_{\Omega}\left[u_{\varepsilon}(x) \times \nabla \varphi(x)\right] \cdot b\left(\varepsilon^{-1} x\right) d \mu_{\varepsilon} .
\end{aligned}
$$

Левая часть этого равенства сходится к нулю в силу условия (ii). Второе слагаемое справа сходится к нулю в силу ограниченности $u_{\varepsilon}$ в $L^{2}\left(\Omega, d \mu_{\varepsilon}\right)$. В результате получим равенство

$$
\int_{\Omega} \int_{\square} \varphi(x) a(y) \cdot u(x, y) d \mu d x=0,
$$

которое с учетом леммы об аппроксимации дает требуемое. Теорема доказана.

9.3. До сих пор на множество $\mathcal{R}$ периодических жестких перемещений никаких ограничений не накладывалось.

Теперь введем основное условие: любой вектор $u \in \mathcal{R}$ допускает единственное представление

$$
u(y)=c+g(y),
$$

где $c$ - постоянный вектор, а $g$ - градиент нуля, $g \in \Gamma(0) \cap \mathcal{R}=\mathcal{R}_{1}$.

Уже отмечалось, что это разложение выполнено для всех примеров, но доказательство для меры $\mu$ общего вида не найдено. Разложение (9.10) играет ту же роль в теории упругости, что и эргодичность (2-связность) меры $\mu$ в скалярных задачах.

Определим некоторые пространства симметрических матриц. Ортогональное разложение (8.1) дает разложение

$$
L^{2}(\Omega \times \square, d x \times d \mu)^{\frac{N(N+1)}{2}}=L^{2}\left(\Omega, \mathcal{V}_{\mathrm{pot}}\right) \oplus L^{2}\left(\Omega, \mathcal{V}_{\mathrm{sol}}\right)
$$

Формально $L^{2}\left(\Omega, \mathcal{V}_{\text {pot }}\right)$ - это совокупность квадратично интегрируемых функций аргумента $x \in \Omega$ со значениями в $\mathcal{V}_{\text {pot. }}$. Однако проще определить $L^{2}\left(\Omega, \mathcal{V}_{\text {pot }}\right)$ как замыкание линейной оболочки матрищ $f(x) e_{y}(\varphi)$, где $f \in C_{0}^{\infty}(\Omega), \varphi \in C_{\mathrm{per}}^{\infty}(\square)^{N}$. Аналогично, $L^{2}\left(\Omega, V_{\mathrm{sol}}\right)$ - это замыкание линейной оболочки матрищ $f(x) b(y)$, где $f \in C_{0}^{\infty}(\Omega), b \in \mathcal{V}_{\text {sol }}$.

Будем изучать последовательность $u_{\varepsilon} \in C^{\infty}(\bar{\Omega})^{N}$, предполагая, что $u_{\varepsilon}, e\left(u_{\varepsilon}\right)$ ограничены в $L^{2}\left(\Omega, d \mu_{\varepsilon}\right)$.

ТЕОРема 9.5. Пусть усредненный тензор невырожден и выполнено основное условие (9.10) на структуру периодических жестких перемещений. Тогда ( с точностью до выделения подпоследовательности)

$$
\begin{gathered}
u_{\varepsilon}(x) \stackrel{2}{\rightarrow} u(x, y)=u_{0}(x)+\chi(x, y), \quad \chi \in L^{2}\left(\Omega, \mathcal{R}_{1}\right), \\
u_{0} \in H^{1}(\Omega)^{N}, \quad e\left(u_{\varepsilon}(x)\right) \stackrel{2}{\rightarrow} e\left(u_{0}(x)\right)+v(x, y), \quad v \in L^{2}\left(\Omega, \mathcal{V}_{\text {pot }}\right) .
\end{gathered}
$$

Кроме того, если с самого начала $u_{\varepsilon} \in C_{0}^{\infty}(\Omega)^{N}$, то $u_{0} \in H_{0}^{1}(\Omega)^{N}$. 
ДОКАЗАТЕЛЬСТВО. Соотношение (9.12) следует из предыдущей теоремы и основного разложения (9.10). Обозначим через $p=p(x, y)$ слабый двухмасштабный предел $e\left(u_{\varepsilon}\right)$,

$$
e\left(u_{\varepsilon}(x)\right) \stackrel{2}{-} p(x, y), \quad p \in L^{2}(\Omega \times \square)^{\frac{N(N+1)}{2}} .
$$

Для $b \in \mathcal{V}_{\mathrm{sol}},\langle b\rangle=\eta, \varphi \in C_{0}^{\infty}(\Omega)$ имеем (см. (3.10), (3.11))

$$
\int_{\Omega} \varphi(x) e\left(u_{\varepsilon}(x)\right) \cdot b\left(\varepsilon^{-1} x\right) d \mu_{\varepsilon}=-\int_{\Omega}\left[u_{\varepsilon}(x) \times \nabla \varphi(x)\right] \cdot b\left(\varepsilon^{-1} x\right) d \mu_{\varepsilon} .
$$

Отсюда по определению двухмасштабной сходимости

$$
\int_{\Omega} \int_{\square} \varphi(x) p(x, y) \cdot b(y) d x d \mu=-\int_{\Omega} \int_{\square}[u(x, y) \times \nabla \varphi(x)] \cdot b(y) d x d \mu .
$$

Соленоидальная матрица $b$ автоматически тангенциальна, а вектор $\chi$ является градиентом нуля. По лемме 5.3

$$
(\chi \times \nabla \varphi) \cdot b=0,
$$

и можно заменить $u(x, y)$ на $u_{0}(x)$ :

$$
\int_{\Omega} \int_{\square} \varphi(x) p(x, y) \cdot b(y) d x d \mu=-\int_{\Omega} \int_{\square}\left[u_{0}(x) \times \nabla \varphi(x)\right] \cdot b(y) d x d \mu .
$$

Поэтому

$$
\int_{\Omega}\left[u_{0} \times \nabla \varphi\right] \cdot \eta d x=-\int_{\Omega} \int_{\square} p(x, y) \cdot \varphi(x) b(y) d x d \mu=-\int_{\Omega} z(x) \varphi(x) d x,
$$

где

$$
z(x)=\int_{\square} p(x, y) \cdot b(y) d \mu, \quad z \in L^{2}(\Omega) .
$$

Отсюда следует, что $e\left(u_{0}\right) \cdot \eta=z \in L^{2}(\Omega)$ в смысле распределений, поэтому

$$
\int_{\Omega} \int_{\square} \varphi(x) p(x, y) \cdot b(y) d x d \mu=\int_{\Omega} e\left(u_{0}(x)\right) \cdot \eta \varphi(x) d x .
$$

Теперь используем невырожденность усредненного тензора. В этом случае (см. предложение 8.1) для любого $\eta \in \mathbb{R}^{\frac{N(N+1)}{2}}$ найдется матрица $b \in \mathcal{V}_{\text {sol }}$ такая, что $\langle b\rangle=\eta$. Поэтому $e\left(u_{0}\right) \in L^{2}(\Omega)$ в смысле распределений и по неравенству Корна $u_{0} \in H^{1}(\Omega)^{N}$. В силу этого теперь имеем

$$
\int_{\Omega} e\left(u_{0}(x)\right) \cdot \eta \varphi(x) d x=\int_{\Omega} \int_{\square} e\left(u_{0}(x)\right) \cdot b(y) \varphi(x) d x d \mu
$$

и соотношение (9.14) можно переписать так:

$$
\int_{\Omega} \int_{\square} \varphi(x)\left[p(x, y)-e\left(u_{0}(x)\right)\right] \cdot b(y) d x d \mu=0 .
$$


Линейная оболочка матрищ $\varphi(x) b(y)$ плотна в $L^{2}\left(\Omega, \mathcal{V}_{\text {sol }}\right)$. Поэтому из ортогонального разложения (9.11) следует $p-e\left(u_{0}\right) \in L^{2}\left(\Omega, \mathcal{V}_{\text {pot }}\right)$ и соотношение $(9.13)$ нашей теоремы доказана.

Допустим теперь, что $u_{\varepsilon} \in C_{0}^{\infty}(\Omega)^{N}$. Продолжим $u_{\varepsilon}$ нулем в более широкую область $\Omega_{1} \supset \bar{\Omega}$. Очевидно, что двухмасштабный предел $u_{\varepsilon}$ в области $\Omega_{1}-$ это продолженный нулем предел в области $\Omega$. Получается, что $u_{0}=0$ вне $\Omega$ и $u_{0} \in H^{1}(\Omega)^{N}$. Отсюда следует (область $\Omega$ липшицева), что $u_{0}$ принадлежит пространству $H_{0}^{1}(\Omega)^{N}$ - замыканию $C_{0}^{\infty}(\Omega)^{N}$ в $H^{1}(\Omega)^{N}$. Теорема полностью доказана.

Эта теорема остается верной и в случае вырожденного тензора $A^{\text {hom }}$, но тогда требуются некоторые изменения в формулировке, которые сейчас укажем.

Было доказано, что $e\left(u_{0}\right) \cdot \eta \in L^{2}(\Omega), \eta=\langle b\rangle$. Отсюда (см. предложение 8.1) $\tilde{e}\left(u_{0}\right) \in L^{2}(\Omega)$, где $\tilde{e}(u)$ - ортогональная проекция $e(u)$ на подпространство $\tilde{L} \subset$ $\mathbb{R}^{\frac{N(N+1)}{2}} ;$ в частности, $A^{\text {hom }} \tilde{e}\left(u_{0}\right)=A^{\text {hom }} e\left(u_{0}\right) \in L^{2}(\Omega)$. Из (9.14) имеем

$$
\begin{aligned}
\int_{\Omega} \int_{\square} \varphi(x) p(x, y) \cdot b(y) d x d \mu & =\int_{\Omega} \tilde{e}\left(u_{0}(x)\right) \cdot \eta \varphi(x) d x \\
& =\int_{\Omega} \int_{\square} \tilde{e}\left(u_{0}(x)\right) \cdot b(y) \varphi(x) d x d \mu,
\end{aligned}
$$

и поэтому $p(x, y)-\tilde{e}\left(u_{0}(x)\right) \in L^{2}\left(\Omega, \mathcal{V}_{\text {pot }}\right)$. Если ввести пространство

$$
\widetilde{\mathcal{H}}^{1}(\Omega)=\left\{u \in L^{2}(\Omega)^{N}: \tilde{e}(u) \in L^{2}(\Omega)^{\frac{N(N+1)}{2}}\right\},
$$

то вместо (9.13) получим

$$
u_{0} \in \widetilde{\mathcal{H}}^{1}(\Omega), \quad e\left(u_{\varepsilon}\right) \stackrel{2}{\rightarrow} \tilde{e}\left(u_{0}\right)+v, \quad v \in L^{2}\left(\Omega, \mathcal{V}_{\text {pot }}\right) .
$$

Наконец, если $u_{\varepsilon} \in C_{0}^{\infty}(\Omega)^{N}$, то $u_{0}$ принадлежит пространству $\widetilde{\mathcal{H}}_{0}^{1}(\Omega)$ - замыканию $C_{0}^{\infty}(\Omega)^{N}$ в $\widetilde{\mathcal{H}}^{1}(\Omega)$.

До сих пор мы рассматривали последовательность $u_{\varepsilon} \in C^{\infty}(\bar{\Omega})^{N}$. Bсе результаты сохранятся, если брать $u_{\varepsilon}$ из пространства $\mathcal{H}^{1}\left(\Omega, d \mu_{\varepsilon}\right)$. Лишь одно требует внимания - условие ограниченности градиентов $e\left(u_{\varepsilon}\right)$. В случае $u_{\varepsilon} \in \mathcal{H}^{1}\left(\Omega, d \mu_{\varepsilon}\right)$ будем говорить, что найдутся такие $e\left(u_{\varepsilon}\right) \in \mathcal{E}\left(u_{\varepsilon}\right)$, что $e\left(u_{\varepsilon}\right)$ ограничены в $L^{2}\left(\Omega, d \mu_{\varepsilon}\right)$.

Выведем из (9.12), (9.13) основную лемму о сходимости моментов, предполагая для упрощения обозначений, что $A^{\text {hom }}$ невырожден.

ЛЕмма 9.6 (о сходимости моментов). Пусть

$\lim _{\varepsilon \rightarrow 0} \int_{\Omega} A_{\varepsilon}(x) e\left(u_{\varepsilon}(x)\right) \cdot e_{y}\left(w\left(\varepsilon^{-1} x\right)\right) \varphi(x) d \mu_{\varepsilon}=0 \quad \forall \varphi \in C_{0}^{\infty}(\Omega), \quad w \in C_{\mathrm{per}}^{\infty}(\square)^{N}$.

Тогда имеет место слабая сходимость

$$
A_{\varepsilon} e\left(u_{\varepsilon}\right) \rightarrow A^{\mathrm{hom}} e\left(u_{0}\right) \quad \text { в } \quad L^{2}\left(\Omega, d \mu_{\varepsilon}\right) .
$$

Кроме того, матрица $v$ из (9.13) есть решение периодической задачи (8.4) $\partial \Omega_{я} \xi=e\left(u_{0}\right)$, m.e. $\operatorname{div}_{y}\left(A(y)\left(e\left(u_{0}\right)+v\right)\right)=0$. 
ДоКАЗАТЕЛЬСТВо. Из свойств (iii), (iv) слабой двухмасштабной сходимости имеем

$$
\begin{aligned}
& A\left(\varepsilon^{-1} x\right) e\left(u_{\varepsilon}\right) \stackrel{2}{\rightarrow} A(y)\left[e\left(u_{0}(x)\right)+v(x, y)\right], \\
& A\left(\varepsilon^{-1} x\right) e\left(u_{\varepsilon}\right) \rightarrow \int_{\square} A(y)\left[e\left(u_{0}(x)\right)+v(x, y)\right] d \mu .
\end{aligned}
$$

В частности, предел (9.15) равен

$$
\int_{\Omega} \int_{\square} A(y)\left[e\left(u_{0}(x)\right)+v(x, y)\right] \varphi(x) \cdot e_{y}(w(y)) d x d y=0 .
$$

Поскольку $\varphi \in C_{0}^{\infty}(\Omega)$ произвольно, то

$$
\int_{\square} A(y)\left[e\left(u_{0}(x)\right)+v(x, y)\right] \cdot e_{y}(w(y)) d \mu=0 \quad \forall w \in C_{\mathrm{per}}^{\infty}(\square)^{N} .
$$

Видим, что $v(x, \cdot)$ есть решение периодической задачи (8.4) для $\xi=e\left(u_{0}\right)$ и по формуле (8.5) выполнено

$$
\int_{\square} A(y)\left[e\left(u_{0}(x)\right)+v(x, y)\right] d \mu=A^{\mathrm{hom}} e\left(u_{0}\right)
$$

Теперь, используя свойство (9.17), получим искомую сходимость (9.16). Лемма доказана.

Любопытно отметить, что в этой лемме $u_{\varepsilon}$ не является решением какого-либо уравнения. Роль “уравнения" играет само дополнительное условие (9.15).

Докажем еше одно свойство, предполагая по-прежнему, что $u_{\varepsilon}, e\left(u_{\varepsilon}\right)$ ограничены в $L^{2}\left(\Omega, d \mu_{\varepsilon}\right)$ и имеет место двухмасштабная сходимость $(9.12),(9.13)$.

ЛЕмма 9.7. Справедливо неравенство

$$
\begin{aligned}
\liminf _{\varepsilon \rightarrow 0} \int_{\Omega} A\left(\varepsilon^{-1} x\right) e\left(u_{\varepsilon}\right) \cdot e\left(u_{\varepsilon}\right) d \mu_{\varepsilon} & \geqslant \int_{\Omega} \int_{\square}\left[A(y)\left(e\left(u_{0}\right)+v\right)\right] \cdot\left[e\left(u_{0}\right)+v\right] d x d \mu \\
& \geqslant \int_{\Omega} A^{\text {hom }} e\left(u_{0}\right) \cdot e\left(u_{0}\right) d x
\end{aligned}
$$

ДокАЗАТЕЛЬСтво. Первое из этих неравенств следует из (9.3). Далее, поскольку $v(x, \cdot) \in \mathcal{V}_{\text {pot }}$, то согласно (8.3)

$$
\int_{\square}\left[A(y)\left(e\left(u_{0}(x)\right)+v(x, y)\right)\right] \cdot\left[e\left(u_{0}(x)\right)+v(x, y)\right] d \mu(y) \geqslant A^{\text {hom }} e\left(u_{0}(x)\right) \cdot e\left(u_{0}(x)\right) .
$$

Лемма доказана. 


\section{$\S 10$. Вывод неклассического усредненного уравнения}

Приведем общую формулировку неклассического принципа усреднения, предполагая для простоты, что усредненный тензор невырожден (см. (2.3)). Наиболее сушественным является разложение (9.10), которое предполагается выполненным.

Теорема 10.1. Пусть $u_{\varepsilon}-$ решения задач Дирихле (1.3), причем последовательность правых частей $f_{\varepsilon}(x)$ ограничена в $L^{2}\left(\Omega, d \mu_{\varepsilon}\right)^{N}$ и $f_{\varepsilon}(x) \stackrel{2}{\rightarrow} f(x, y)$. Тогда имеет место слабая двухмасшабная сходимость (9.12), (9.13). При этом вектор-функция $u=u(x, y)$ из (9.12) есть решение усредненной задачи (2.10), а матрица $v(x, y)$ из (9.13) - решение периодической задачи (8.4) $\partial \Omega я \xi=e\left(u_{0}\right)$, m.e. $\operatorname{div}_{y}\left(A(y)\left(e\left(u_{0}\right)+v\right)\right)=0$.

Кроме того, если сходимость правых частей является сильной двухмасштабной, $f_{\varepsilon}(x) \stackrel{2}{\longrightarrow} f(x, y)$, то сходимость в (9.12) и в (9.13) становится также сильной двухмасштабной.

ДоКАЗАТЕЛЬСтво. Из интегрального тождества (1.4) следует, что $u_{\varepsilon}, e\left(u_{\varepsilon}\right)$ ограничены в $L^{2}\left(\Omega, d \mu_{\varepsilon}\right)$. Без потери обшности считаем, что имеет место (9.12), (9.13).

Тождество (2.10) распадается на два соотношения:

$$
\begin{gathered}
-\operatorname{div} A^{\mathrm{hom}} e\left(u_{0}\right)+\langle u\rangle=\langle f\rangle, \\
u(x, \cdot)-f(x, \cdot) \perp \mathcal{R}_{1} .
\end{gathered}
$$

Первое получится, если в $(2.10)$ взять $\varphi=\varphi_{0} \in C_{0}^{\infty}(\Omega)^{N}$, а второе - если взять $\varphi=\varphi_{1} \in L^{2}\left(\Omega, \mathcal{R}_{1}\right)$. В том случае, когда $f$ не зависит от $y$, эти соотношения совпадают с (2.6) и (2.7).

Вывод усредненного уравнения разобьем на два шага.

Шаг 1. Взяв в тождестве (1.4) пробную вектор-функцию

$$
\psi(x)=\varepsilon \varphi(x) w\left(\varepsilon^{-1} x\right), \quad \varphi \in C_{0}^{\infty}(\Omega), \quad w \in C_{\mathrm{per}}^{\infty}(\square)^{N},
$$

получим

$$
\begin{gathered}
\int_{\Omega} \varphi A_{\varepsilon} e\left(u_{\varepsilon}\right) \cdot e_{y}(w) d \mu_{\varepsilon}+\varepsilon \int_{\Omega} A_{\varepsilon} e\left(u_{\varepsilon}\right) \cdot[\nabla \varphi \times w] d \mu_{\varepsilon} \\
+\varepsilon \int_{\Omega} u_{\varepsilon} \cdot \varphi w d \mu_{\varepsilon}=\varepsilon \int_{\Omega} f_{\varepsilon} \cdot \varphi w d \mu_{\varepsilon} .
\end{gathered}
$$

Все слагаемые, кроме первого, сходятся к нулю при $\varepsilon \rightarrow 0$. Тогда сходится к нулю и первое слагаемое. Это означает, что выполнено условие (9.15) леммы о сходимости моментов, в частности имеет место слабая сходимость моментов (9.16). Последнее позволяет перейти к пределу в интегральном тождестве (1.4) и получить тождество

$$
\begin{array}{r}
\int_{\Omega} A^{\text {hom }} e\left(u_{0}\right) \cdot e(\psi) d x+\int_{\Omega} \int_{\square} u(x, y) \cdot \psi(x) d x d \mu \\
=\int_{\Omega} \int_{\square} f(x, y) \cdot \psi(x) d x d \mu, \quad \psi \in C_{0}^{\infty}(\Omega)^{N},
\end{array}
$$


эквивалентное (10.1).

Шаг 2. Сначала заметим, что в тождестве (1.4) в качестве пробной векторфункции $\psi$ можно взять вектор-функцию из $\mathcal{H}_{0}^{1}\left(\Omega, d \mu_{\varepsilon}\right)$, причем в качестве $e(\psi)$ можно рассматривать любой ее симметрический градиент. Возьмем пробную вектор-функцию из (7.1). Так как $0 \in \mathcal{E}(\psi)$ (по лемме 7.2), то интегральное тождество (1.4) принимает вид

$$
\int_{\Omega} u_{\varepsilon}(x) \cdot \varphi(x) g\left(\varepsilon^{-1} x\right) d \mu_{\varepsilon}=\int_{\Omega} f_{\varepsilon}(x) \cdot \varphi(x) g\left(\varepsilon^{-1} x\right) d \mu_{\varepsilon} .
$$

Предельным переходом получим соотношение

$$
\int_{\Omega} \int_{\square}[u(x, y)-f(x, y)] \cdot \varphi(x) g(y) d x d \mu=0,
$$

эквивалентное (10.2). Усредненное уравнение (2.10) получено.

Рассмотрим случай сильной сходимости $f_{\varepsilon} \stackrel{2}{\longrightarrow} f$. Из тождества (1.4) имеем

$$
\int_{\Omega} A_{\varepsilon} e\left(u_{\varepsilon}\right) \cdot e\left(u_{\varepsilon}\right) d \mu_{\varepsilon}+\int_{\Omega}\left|u_{\varepsilon}\right|^{2} d \mu_{\varepsilon}=\int_{\Omega} f_{\varepsilon} \cdot u_{\varepsilon} d \mu_{\varepsilon} .
$$

Поскольку слабая сходимость $u_{\varepsilon}(x) \stackrel{2}{\rightarrow} u(x, y)$ уже доказана, то

$$
\lim _{\varepsilon \rightarrow 0} \int_{\Omega} A_{\varepsilon} e\left(u_{\varepsilon}\right) \cdot e\left(u_{\varepsilon}\right) d \mu_{\varepsilon}+\lim _{\varepsilon \rightarrow 0} \int_{\Omega}\left|u_{\varepsilon}\right|^{2} d \mu=\int_{\Omega} \int_{\square} f \cdot u d x d \mu .
$$

С другой стороны, справедливо энергетическое равенство (3.4); поэтому из свойств полунепрерывности $(9.4),(9.18)$ получаем равенства

$$
\begin{aligned}
\lim _{\varepsilon \rightarrow 0} \int_{\Omega}\left|u_{\varepsilon}\right|^{2} d \mu_{\varepsilon} & =\int_{\Omega} \int_{\square}|u|^{2} d x d \mu \\
\lim _{\varepsilon \rightarrow 0} \int_{\Omega} A_{\varepsilon} e\left(u_{\varepsilon}\right) \cdot e\left(u_{\varepsilon}\right) d \mu_{\varepsilon} & =\int_{\Omega} \int_{\square} A(y)\left[e\left(u_{0}\right)+v\right] \cdot\left[e\left(u_{0}+v\right)\right] d x d \mu
\end{aligned}
$$

Из (10.3) и (9.12) имеем сильную сходимость

$$
u_{\varepsilon}(x) \stackrel{2}{\longrightarrow} u(x, y)=u_{0}(x)+\chi(x, y)
$$

Далее, двухмасштабная сходимость (9.13) влечет сходимость

$$
A^{\frac{1}{2}}\left(\varepsilon^{-1} x\right) e\left(u_{\varepsilon}(x)\right) \stackrel{2}{\rightarrow} A^{\frac{1}{2}}(y)\left[e\left(u_{0}(x)\right)+v(x, y)\right]
$$

причем эта сходимость оказывается сильной благодаря (10.4). Отсюда (используя (9.5)) легко получить, что

$$
e\left(u_{\varepsilon}(x)\right) \stackrel{2}{\longrightarrow} e\left(u_{0}(x)\right)+v(x, y) .
$$

Итак, доказана сильная двухмасштабная сходимость решений и тензоров деформации. Полученные соотношения (10.5), (10.6) представляют собой общую 
форму так называемой теоремы о корректоре. Рассмотрим некоторые частные варианты.

Допустим, что $f(x, y)=f(x) \in C^{\infty}(\bar{\Omega})^{N}$. Из (2.9) легко видеть, что слагаемое $\chi(x, y)$ имеет структуру, предусмотренную в свойстве $(9.6)$ сильной двухмасштабной сходимости. Поэтому сходимость (10.5) принимает вид

$$
\lim _{\varepsilon \rightarrow 0} \int_{\Omega}\left|u_{\varepsilon}(x)-u_{0}(x)-\chi\left(x, \frac{x}{\varepsilon}\right)\right|^{2} d \mu_{\varepsilon}=0 .
$$

Тем самым доказана сходимость (2.4).

Аналогично можно упростить и сходимость (10.6).

Для $v=v(x, y)$ из (10.6) справедливо представление $v(x, y)=\hat{v}\left(y, e_{x}\left(u_{0}(x)\right)\right)$, где $\hat{v}(y, \xi)$ - линейно зависяшее от $\xi$ решение периодической задачи (8.4). Если $u_{0} \in C^{1}(\bar{\Omega})^{N}$, то

$$
\lim _{\varepsilon \rightarrow 0} \int_{\Omega}\left|e\left(u_{\varepsilon}(x)\right)-e\left(u_{0}(x)\right)-v\left(x, \frac{x}{\varepsilon}\right)\right|^{2} d \mu_{\varepsilon}=0 .
$$

При дополнительных условиях можно пойти и дальше.

Пусть выполнено неравенство Корна (8.7). Запишем решения $\hat{v}(y, \xi)$ периодической задачи (8.4) в виде $\hat{v}(y, \xi)=\nabla_{y} w(y, \xi)$ (см. представление $\left.(8.8)\right)$ и положим

$$
u_{1}(x, y)=w\left(y, e_{x}\left(u_{0}(x)\right)\right)
$$

Считая, что $u_{0} \in C^{2}(\bar{\Omega})^{N}$, из (10.8) получим соотношение

$$
\lim _{\varepsilon \rightarrow 0} \int_{\Omega}\left|e\left(u_{\varepsilon}(x)-u_{0}(x)-\varepsilon u_{1}\left(x, \frac{x}{\varepsilon}\right)\right)\right|^{2} d \mu_{\varepsilon}=0,
$$

совпадающее по форме с классической теоремой о корректоре.

\section{§ 11. Обобщенная двухмасштабная сходимость}

11.1. Для изучения масштабного эффекта в случае тонких сеток и других аналогичных моделей потребуется несколько обобщить теорию двухмасштабной сходимости.

Ранее периодическая мера $\mu$ была фиксирована и мы вводили двухмасштабную сходимость с помощью скейлинг-меры $\mu_{\varepsilon}$, определенной равенством (1.1).

Теперь рассмотрим семейство периодических нормированных мер $\mu^{h}, \mu^{h} \rightarrow \mu$ при $h \rightarrow 0$. При фиксированном $h$ рассмотрим скейлинг-меру $\mu_{\varepsilon}^{h}$, определенную равенством (1.1), затем положим, что $h=h(\varepsilon) \rightarrow 0$ при $\varepsilon \rightarrow 0$.

СвОЙСТВО СРЕДНЕГО ЗНАЧЕНИЯ. Пусть $a^{h} \in L_{\mathrm{per}}^{1}\left(\square, d \mu^{h}\right), a^{h} \geqslant 0$,

$$
\lim _{h \rightarrow 0} \int_{\square} a^{h} d \mu^{h}=\alpha<\infty .
$$

Если область $\Omega$ ограничена и измерима по Жордану (т.е. $\partial \Omega$ имеет нулевой лебегов обгем), то

$$
\lim _{\varepsilon \rightarrow 0} \int_{\Omega} \varphi(x) a^{h}\left(\varepsilon^{-1} x\right) d \mu_{\varepsilon}^{h}=\alpha \int_{\Omega} \varphi(x) d x, \quad \varphi \in C(\bar{\Omega}) .
$$


ДокаЗАтельСтво. Разобьем $\mathbb{R}^{N}$ на полуоткрытые кубы $[0, \varepsilon)^{N}+\varepsilon n, n$ - целочисленный вектор, и обозначим отдельный куб символом $\varepsilon \square_{j}$. Имеем

$$
\begin{aligned}
\int_{\Omega} \varphi(x) a^{h}\left(\varepsilon^{-1} x\right) d \mu_{\varepsilon}^{h}= & \sum \int_{\varepsilon \square_{j}} \varphi(x) a^{h}\left(\varepsilon^{-1} x\right) d \mu_{\varepsilon}^{h} \\
& +\sum \int_{\Omega \cap \varepsilon \square_{j}} \varphi(x) a^{h}\left(\varepsilon^{-1} x\right) d \mu_{\varepsilon}^{h},
\end{aligned}
$$

где первая сумма берется по кубам, целиком лежащим в $\Omega$, а вторая - по приграничным кубам. По теореме о среднем

$$
\int_{\varepsilon \square_{j}} \varphi(x) a^{h}\left(\varepsilon^{-1} x\right) d \mu_{\varepsilon}^{h}=\varphi\left(x^{j}\right) \int_{\varepsilon \square_{j}} a^{h}\left(\varepsilon^{-1} x\right) d \mu_{\varepsilon}^{h}=\varepsilon^{N} \varphi\left(x^{j}\right) \int_{\square_{j}} a^{h}(y) d \mu^{h}(y),
$$

где $x^{j}$ - точка из $\varepsilon \square_{j}$. В результате получим

$$
\left|\int_{\Omega} \varphi(x) a^{h}\left(\varepsilon^{-1} x\right) d \mu_{\varepsilon}^{h}-\varepsilon^{N} \alpha^{h} \sum \varphi\left(x^{j}\right)\right| \leqslant \max |\varphi| \alpha^{h} \varepsilon^{N} D(\varepsilon),
$$

где $\alpha^{h}=\int_{\square} a^{h} d \mu^{h}, D(\varepsilon)$ - число приграничных кубов, $\varepsilon^{N} D(\varepsilon) \rightarrow 0$ в силу измеримости по Жордану. Поскольку (см. (11.1))

$$
\lim _{\varepsilon \rightarrow 0} \varepsilon^{N} \alpha^{h} \sum \varphi\left(x^{j}\right)=\alpha \int_{\Omega} \varphi(x) d x
$$

то равенство (11.2) доказано. Из него получаем

$$
\lim _{\varepsilon \rightarrow 0} \int_{\Omega} \varphi(x) b\left(\varepsilon^{-1} x\right) d \mu_{\varepsilon}^{h}=\int_{\Omega} \int_{\square} \varphi(x) b(y) d \mu(y), \quad \varphi \in C_{0}^{\infty}(\Omega), \quad b \in C_{\mathrm{per}}^{\infty} .
$$

(Для доказательства этого равенства достаточно записать функцию $b$ как разность двух положительных функций и применить равенство (11.2).)

Пусть $v_{\varepsilon, h}$ - ограниченная последовательность в $L^{2}\left(\Omega, d \mu_{\varepsilon}^{h}\right)$.

ОПРЕДЕлЕниЕ 11.1. Выполнено $v_{\varepsilon, h}(x) \stackrel{2}{-} v(x, y) \in L^{2}(\Omega \times \square, d x \times d \mu)$, если

$$
\lim _{\varepsilon \rightarrow 0} \int_{\Omega} \varphi(x) b\left(\varepsilon^{-1} x\right) v_{\varepsilon, h}(x) d \mu_{\varepsilon}^{h}=\int_{\Omega} \int_{\square} \varphi(x) b(y) v(x, y) d x d \mu
$$

для любых $\varphi \in C_{0}^{\infty}(\Omega), b \in C_{\text {per }}^{\infty}(\square)$.

Имеет место следуюший принцип выбора.

Теорема 11.2. Ограниченная в $L^{2}\left(\Omega, d \mu_{\varepsilon}^{h}\right)$ последовательность компактна в смысле слабой двухмасштабной сходимости. 
ДоказАТЕЛЬСТво. Множество пробных функций $\Phi(x, y)=\varphi_{i}(x) b_{i}(y), \varphi_{i} \in$ $C_{0}^{\infty}(\Omega), b_{i} \in C_{\mathrm{per}}^{\infty}(\square)$, сепарабельно относительно нормы $\sup _{\Omega \times \square}|\Phi|$. Выбирая счетное всюду плотное множество $K$ таких функций, диагональным методом выделим подпоследовательность $\varepsilon_{k} \rightarrow 0$ такую, что предел

$$
\lim _{\varepsilon_{k} \rightarrow 0} \int_{\Omega} \Phi\left(x, \varepsilon^{-1} x\right) v_{\varepsilon, h}(x) d \mu_{\varepsilon}^{h}=l(\Phi)
$$

сушествует для любой $\Phi \in K$. Но тогда этот предел существует и для любой функции $\Phi$. Далее, по неравенству Коши-Буняковского имеем

$$
\begin{aligned}
\left(\int_{\Omega} \Phi\left(x, \varepsilon^{-1} x\right) v_{\varepsilon, h}(x) d \mu_{\varepsilon}^{h}\right)^{2} & \leqslant \int_{\Omega} v_{\varepsilon, h}^{2} d \mu_{\varepsilon}^{h} \int_{\Omega} \Phi^{2}\left(x, \varepsilon^{-1} x\right) d \mu_{\varepsilon}^{h} \\
& \leqslant C \int_{\Omega} \Phi^{2}\left(x, \varepsilon^{-1} x\right) d \mu_{\varepsilon}^{h}
\end{aligned}
$$

Отсюда по свойству среднего значения получаем

$$
|l(\Phi)|^{2} \leqslant C \int_{\Omega} \int_{\square} \Phi^{2}(x, y) d x d \mu .
$$

Тогда по теореме Рисса линейный функционал $l(\Phi)$ допускает следуюшее представление:

$$
l(\Phi)=\int_{\Omega} \int_{\square} \Phi(x, y) v(x, y) d x d \mu, \quad v \in L^{2}(\Omega \times \square) .
$$

Теорема доказана.

Аналогично вводится понятие сильной двухмасштабной сходимости.

ОПРЕДЕЛЕНИЕ 11.3. Выполнено $v_{\varepsilon, h} \stackrel{2}{\longrightarrow} v \in L^{2}(\Omega \times \square, d x \times d \mu)$, если

$$
\lim _{\varepsilon \rightarrow 0} \int_{\Omega} v_{\varepsilon, h}(x) z_{\varepsilon, h}(x) d \mu_{\varepsilon}^{h}=\int_{\Omega} \int_{\square} v(x, y) z(x, y) d x d \mu,
$$

как только $z_{\varepsilon, h}(x) \stackrel{2}{\rightarrow} z(x, y)$.

11.2. В определении 11.1 слабой двухмасштабной сходимости использован минимально узкий класс пробных функций. Требуется расширить этот класс с сохранением сходимости (11.3); в частности, функцию $b$ нужно сделать зависящей от $h$.

С этой целью напомним понятия слабой и сильной сходимости в $L^{2}\left(\square, d \mu^{h}\right)[17]$.

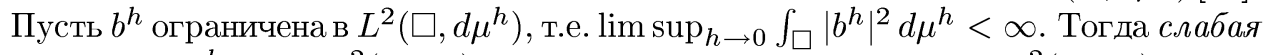
сходимость $b^{h} \rightarrow b$ в $L^{2}(\square, d \mu)$ означает по определению, что $b \in L^{2}(\square, d \mu)$ и

$$
\lim _{h \rightarrow 0} \int_{\square} b^{h} \varphi d \mu^{h}=\int_{\square} b \varphi d \mu \quad \forall \varphi \in C_{\mathrm{per}}^{\infty}(\square) .
$$

Сильная сходимость $b^{h} \longrightarrow b$ означает, что $b \in L^{2}(\square, d \mu)$ и

$$
\lim _{h \rightarrow 0} \int_{\square} b^{h} g^{h} d \mu^{h}=\int_{\square} b g d \mu, \quad \text { как только } g^{h} \rightarrow g .
$$


Установим некоторые элементарные свойства сходимости, прежде всего - полунепрерывность снизу:

$$
\text { если } b^{h}-b, \quad \text { то } \lim _{h \rightarrow 0} \int_{\square}\left|b^{h}\right|^{2} d \mu^{h} \geqslant \int_{\square}|b|^{2} d \mu .
$$

Действительно, для $\varphi \in C_{\text {per }}^{\infty}$ имеем

$$
\begin{gathered}
\int_{\square}\left|b^{h}\right|^{2} d \mu^{h} \geqslant 2 \int_{\square} b^{h} \varphi d \mu^{h}-\int_{\square} \varphi^{2} d \mu^{h}, \\
\lim _{h \rightarrow 0} \int_{\square}\left|b^{h}\right|^{2} d \mu^{h} \geqslant 2 \int_{\square} b \varphi d \mu-\int_{\square} \varphi^{2} d \mu .
\end{gathered}
$$

Последнее неравенство по непрерывности выполнено для $\varphi \in L^{2}(\square, d \mu)$, и, взяв $\varphi=b$, получим искомое свойство.

ПрЕДЛОЖЕНИЕ 11.4. і) Сильная сходимость $b^{h} \longrightarrow b$ складывается из слабой $b^{h} \rightarrow b$ и соотношения

$$
\lim _{h \rightarrow 0} \int_{\square}\left|b^{h}\right|^{2} d \mu^{h}=\int_{\square}|b|^{2} d \mu .
$$

ii) $E c л и b^{h} \longrightarrow b$, то для любого $\delta>0$ найдется функиия $\tilde{b} \in C_{\mathrm{per}}^{\infty}(\square)$ такая, чmo

$$
\begin{array}{r}
\int_{\square}|b-\tilde{b}|^{2} d \mu \leqslant \delta, \\
\lim _{h \rightarrow 0} \int_{\square}\left|b^{h}-\tilde{b}\right|^{2} d \mu^{h} \leqslant \delta .
\end{array}
$$

ДоКАЗАТЕЛЬСТво. Очевидно, что сильная сходимость влечет слабую сходимость и равенство (11.7). Теперь в обратную сторону: вьведем сильную сходимость $b^{h} \longrightarrow b$ из слабой и равенства (11.7). Пусть $h_{k} \rightarrow 0$ и сушествуют пределы

$$
\lim _{h_{k} \rightarrow 0} \int_{\square} b^{h} g^{h} d \mu^{h}=\alpha, \quad \lim _{h_{k} \rightarrow 0} \int_{\square}\left|g^{h}\right|^{2} d \mu^{h}=\beta .
$$

Требуется проверить равенство $\alpha=\int_{\square} b g d \mu$ (см. (11.5)). По свойству полунепрерывности снизу

$$
\begin{aligned}
\lim _{h_{k} \rightarrow 0} \int_{\square}\left(b^{h}+t g^{h}\right)^{2} d \mu^{h} & =\lim _{h_{k} \rightarrow 0} \int_{\square}\left|b^{h}\right|^{2} d \mu^{h}+2 t \alpha+t^{2} \beta \geqslant \int_{\square}(b+t g)^{2} d \mu \\
& =\int_{\square}|b|^{2} d \mu+2 t \int_{\square} b g d \mu+t^{2} \int_{\square}|g|^{2} d \mu .
\end{aligned}
$$

С учетом (11.7) получим неравенство

$$
2 t \alpha+t^{2} \beta \geqslant 2 t \int_{\square} b g d \mu+t^{2} \int_{\square}|g|^{2} d \mu \quad \forall t \in \mathbb{R}^{1} .
$$

Отсюда $\alpha=\int_{\square} b g d \mu$, и сильная сходимость $b^{h} \longrightarrow b$ проверена. 
Для доказательства iі) найдем функцию $\tilde{b} \in C_{\text {per }}^{\infty}$, удовлетворяющую (11.8). Тогда

$$
J_{h}=\int_{\square}\left|b^{h}-\tilde{b}\right|^{2} d \mu^{h}=\int_{\square}\left(\left|b^{h}\right|^{2}-2 b^{h} \tilde{b}+|\tilde{b}|^{2}\right) d \mu^{h} .
$$

Согласно (11.7), (11.4) имеем

$$
\lim _{h \rightarrow 0} J_{h}=\int_{\square}\left(|b|^{2}-2 b \tilde{b}+|\tilde{b}|^{2}\right) d \mu=\int_{\square}(b-\tilde{b})^{2} d \mu \leqslant \delta,
$$

и соотношение (11.9) доказано.

Докажем следуюшую лемму о расширении класса пробных функций, участвуюших в соотношении (11.3).

Лемма 11.5. Пусть $v_{\varepsilon, h}(x) \stackrel{2}{-} v(x, y)$ в смислле определения $11.1 u b^{h} \longrightarrow b$ в $L^{2}\left(\square, d \mu^{h}\right)$. Тогда

$$
\lim _{\varepsilon \rightarrow 0} \int_{\Omega} \varphi(x) b^{h}\left(\varepsilon^{-1} x\right) v_{\varepsilon, h}(x) d \mu_{\varepsilon}^{h}=\int_{\Omega} \int_{\square} \varphi(x) b(y) v(x, y) d x d \mu .
$$

ДокАЗАТЕЛЬСтво. Из (11.2), (11.9) следует, что

$$
\int_{\Omega}\left|b^{h}\left(\varepsilon^{-1} x\right)-\tilde{b}\left(\varepsilon^{-1} x\right)\right|^{2} d \mu_{\varepsilon}^{h} \leqslant 2|\Omega| \delta
$$

при достаточно малом $h$. Поэтому

$$
\begin{aligned}
& \left|\int_{\Omega} \varphi(x)\left[b^{h}\left(\varepsilon^{-1} x\right)-\tilde{b}\left(\varepsilon^{-1} x\right)\right] v_{\varepsilon, h}(x) d \mu_{\varepsilon}^{h}\right| \\
& \leqslant c\left(\int_{\Omega}\left|b^{h}\left(\varepsilon^{-1} x\right)-\tilde{b}\left(\varepsilon^{-1} x\right)\right|^{2} d \mu_{\varepsilon}^{h}\right)^{\frac{1}{2}} \leqslant c_{1} \sqrt{\delta} .
\end{aligned}
$$

Кроме того, из определения 11.1 и оценки (11.8) получаем

$$
\begin{gathered}
\lim _{\varepsilon \rightarrow 0} \int_{\Omega} \varphi(x) v_{\varepsilon, h}(x) \tilde{b}\left(\varepsilon^{-1} x\right) d \mu_{\varepsilon}^{h}=\int_{\Omega} \int_{\square} \varphi(x) v(x, y) \tilde{b}(y) d x d \mu, \\
\left|\int_{\Omega} \int_{\square} \varphi(x) v(x, y)(b(y)-\tilde{b}(y)) d x d \mu\right| \leqslant c \sqrt{\delta} .
\end{gathered}
$$

Отсюда следует (11.10). Лемма доказана. 
ПРЕДЛОЖЕнИЕ 11.6. Пусть $v_{\varepsilon, h} \stackrel{2}{\longrightarrow} v=v(x, y) . \quad$ Ecлu $v(x, y)=\sum \varphi_{i}(x) \times$ $b_{i}(y), \quad \varphi_{i} \in C(\bar{\Omega}), \quad b_{i} \in L_{\mathrm{per}}^{2}(\square, d \mu), m o$

$$
\lim _{\varepsilon \rightarrow 0} \int_{\Omega}\left|v_{\varepsilon, h}(x)-v^{h}\left(x, \varepsilon^{-1} x\right)\right|^{2} d \mu_{\varepsilon}^{h}=0,
$$

əде $v^{h}(x, y)=\sum \varphi_{i}(x) b_{i}^{h}(y), \quad b_{i}^{h} \longrightarrow b_{i}$ в $L^{2}\left(\square, d \mu^{h}\right)$.

Перейдем к исследованию вопросов, связанных с одновременным рассмотрением последовательности вектор-функций $u_{\varepsilon, h}$ и соответствуюших тензоров деформации $e\left(u_{\varepsilon, h}\right)$, т.е. получим теоремы, аналогичные теоремам 9.4 и 9.5. Доказательства этих теорем полностью сохраняются при выполнении некоторых условий аппроксимативного характера, из которых наиболее важно следующее: для любой матрицы $b \in \mathcal{V}_{\text {sol }}(\square, d \mu)$ найдется $b^{h} \in \mathcal{V}_{\text {sol }}\left(\square, d \mu^{h}\right)$ такая, что $b^{h} \longrightarrow b$ (сильная аппроксимируемость соленоидальных матрищ). Для различных сингулярных структур (и их аппроксимаций) такого рода свойства нужно специально доказывать. Отложим обшие формулировки до $\S 16$ и перейдем к модельному примеру тонких сеток, где вопросы аппроксимации решаются очень просто.

\section{§ 12. Тонкие периодические сетки}

Рассмотрим модельный пример тонких периодических сеток. Меры $\mu^{h}$ и $\mu$ определены в п. 3.1. Напомним, что бесконечно тонкие стержни (на которых сосредоточена мера $\mu$ ) являются продольными осями симметрии для соответствующих $h$-стержней (см. рис. 7).

Условимся определенным образом продолжать периодическую функцию $b \in$ $L^{2}(\square, d \mu)$ на носитель меры $\mu^{h}$. Рассмотрим один бесконечно тонкий стержень и продолжим функцию $b$ как постоянную в поперечном направлении на $h$-стержень, причем вне данного $h$-стержня полагаем ее равной нулю. После этого рассмотрим сумму таких продолжений по всем четырем $h$-стержням. Результат обозначим символом $b^{h}$, а само продолжение назовем естественны.м.

Очевидно, что естественное продолжение периодично, принадлежит $L^{2}\left(\square, d \mu^{h}\right)$ и сохраняет среднее значение: $\int_{\square} b^{h} d \mu^{h}=\int_{\square} b d \mu$.

Несложными вычислениями получим

$$
b^{h} \rightarrow b, \quad \lim _{h \rightarrow 0} \int_{\square}\left|b^{h}\right|^{2} d \mu^{h}=\int_{\square}|b|^{2} d \mu .
$$

Поэтому (см. предложение 11.4)

$$
b^{h} \longrightarrow b \text { в } L^{2}\left(\square, d \mu^{h}\right)
$$

(сильная сходимость естественных продолжений); в частности, справедливо предельное соотношение (11.10).

Другое важное свойство естественного продолжения - это сохранение соленоидальности. Имеет место следующее простое утверждение.

ПРЕДЛОЖЕНИЕ 12.1. Естественное продолжение матриць $b \in \mathcal{V}_{\mathrm{sol}}(\square, d \mu)$ будет соленоидальной матричей относительно меры $\mu^{h}$, т.е. будет принадлежать $\mathcal{V}_{\mathrm{sol}}\left(\square, d \mu^{h}\right)$. Кроме того, для любого вектора $a \in L^{2}(\square, d \mu)^{2}, a \perp \mathcal{R}$, 
найдется симметрическая матрица $b \in L^{2}(\square, d \mu)^{3}$ такая, что $a=\operatorname{div} b$. При әтом для естественных продолжений матрицы $b$ и вектора а имеет место соотношение $a^{h}=\operatorname{div} b^{h}$ по отношению $\kappa$ мере $\mu^{h}$, m.e

$$
\int_{\square} a^{h} \cdot \varphi d \mu^{h}=-\int_{\square} b^{h} \cdot e(\varphi) d \mu^{h}, \quad \varphi \in C_{\mathrm{per}}^{\infty}(\square)^{2}, \quad \varphi=\left(\varphi^{1}, \varphi^{2}\right) .
$$

ДоКАЗАТЕЛЬСТво. Воспользуемся принципом расщепления (6.9). Из него следует, что $a=\operatorname{div} b$ по мере $\mu$ тогда и только тогда, когда $a=\operatorname{div} b$ по мерам $\mu_{1}, \mu_{2}$, $\mu_{3}, \mu_{4}$.

Рассмотрим меру $\mu_{1}$, отвечающую периодической системе горизонтальных стержней (см. рис. 4). Из условия $a \perp \mathcal{R}$ следует, что

$$
a=\left(a_{1}, 0\right), \quad \int_{I_{1}} a_{1} d x_{1}=0
$$

Соотношение $\operatorname{div} b=a$ (по мере $\left.\mu_{1}\right)$ справедливо для матрищы

$$
b=b\left(x_{1}\right)=\left(\begin{array}{cc}
b_{11}\left(x_{1}\right) & 0 \\
0 & 0
\end{array}\right), \quad b_{11}\left(x_{1}\right)=\int_{0}^{x_{1}} a_{1}(t) d t .
$$

Рассмотрим $a=a\left(x_{1}\right), b=b\left(x_{1}\right)$ на полосе $I_{1}^{h}=I_{1} \times[-h, h]$. Очевидно, что

$$
\int_{I_{1}^{h}} b_{11}\left(x_{1}\right) \frac{\partial \varphi^{1}}{\partial x_{1}}\left(x_{1}, x_{2}\right) d x_{1} d x_{2}=-\int_{I_{1}^{h}} a_{1}\left(x_{1}\right) \varphi^{1}\left(x_{1}, x_{2}\right) d x_{1} d x_{2}, \quad \varphi^{1} \in C_{\mathrm{per}}^{\infty}(\square) .
$$

Вне первого $h$-стрежня $a$ и $b$ считаем равными нулю. Тогда (12.2) может быть записано в виде (12.1). Действуя подобным образом для каждого из остальных стержней и складывая равенства типа (12.1), получим искомое равенство. Предложение доказано.

Теперь убедимся в том, что все результаты $\S 9$ о двухмасштабной сходимости, связанные с мерой $\mu_{\varepsilon}$, сохраняются и в случае меры $\mu_{\varepsilon}^{h}$.

ТЕОРема 12.2. Пусть выполнены условия:

(i) $u_{\varepsilon, h}(x) \stackrel{2}{\rightarrow} u(x, y)$;

(ii) $\varepsilon e\left(u_{\varepsilon, h}\right) \rightarrow 0$ в $L^{2}\left(\Omega, d \mu_{\varepsilon}^{h}\right)$.

Тогда $u(x, y) \in L^{2}(\Omega, \mathcal{R})$.

ДокАЗАТЕльСтво. Для $a \in L^{2}(\square, d \mu)^{2}, a \perp \mathcal{R}$, найдем симметрическую матрицу $b \in L^{2}(\square, d \mu)$ такую, что $\operatorname{div} b=a$. Тогда для естественных продолжений вьполнено (12.1), и поэтому справедливо тождество

$$
\begin{gathered}
\varepsilon \int_{\Omega} e\left(u_{\varepsilon, h}(x)\right) \cdot b^{h}\left(\varepsilon^{-1} x\right) \varphi d \mu_{\varepsilon}^{h}+\varepsilon \int_{\Omega}\left[u_{\varepsilon, h}(x) \times \nabla \varphi(x)\right] \cdot b^{h}\left(\varepsilon^{-1} x\right) d \mu_{\varepsilon}^{h} \\
=-\int_{\Omega} \varphi(x) a^{h}\left(\varepsilon^{-1} x\right) \cdot u_{\varepsilon, h}(x) d \mu_{\varepsilon}^{h}, \quad \varphi \in C_{0}^{\infty}(\Omega) .
\end{gathered}
$$


Первое слагаемое в левой части стремится к нулю в силу условия (ii). Второе также имеет нулевой предел. Поэтому

$$
\lim _{\varepsilon \rightarrow 0} \int_{\Omega} \varphi(x) a^{h}\left(\varepsilon^{-1} x\right) \cdot u_{\varepsilon, h}(x) d \mu_{\varepsilon}^{h}=0 .
$$

С другой стороны, этот же предел (согласно лемме 11.5) равен

$$
\int_{\Omega} \int_{\square} \varphi(x) a(y) \cdot u(x, y) d x d \mu=0 \quad \forall a \perp \mathcal{R} .
$$

Отсюда следует, что $u \in L^{2}(\Omega, \mathcal{R})$. Теорема доказана.

Видим, что доказательство, по существу, ничем не отличается от того случая, когда мера $\mu$ не зависела от $h$. Аналогично устанавливается основная теорема 9.5: достаточно вместо вектора $b \in \mathcal{V}_{\text {sol }}(\square, d \mu)$ взять его естественное продолжение $b^{h} \in \mathcal{V}_{\text {sol }}\left(\square, d \mu^{h}\right)$. Лемма 9.6 о сходимости моментов является следствием теоремы 9.5.

Располагая аналогами теорем 9.5 и 9.6 переходим к задаче (3.1) и теореме типа теоремы 10.1. В полном объеме эту теорему повторить не представляется возможным, но элементарньй шаг 1, посвяшенный выводу предельного соотношения (10.1), остается в силе, поскольку опирается только на лемму о сходимости моментов. Таким образом, справедливо соотношение (10.1), совпадающее в данном случае с (2.6), и мы приходим к следующей теореме.

TЕОРема 12.3. Пусть $h(\varepsilon) \rightarrow 0$ произвольно и $u_{\varepsilon, h}$ - последовательность из $\mathcal{H}_{0}^{1}\left(\Omega, d \mu_{\varepsilon}^{h}\right)$ такая, что $u_{\varepsilon, h}$, e( $\left.u_{\varepsilon, h}\right)$ ограничены в $L^{2}\left(\Omega, d \mu_{\varepsilon}^{h}\right)$. Тогда (c точностью до выделения подпоследовательности)

$$
\begin{gathered}
u_{\varepsilon, h}(x) \stackrel{2}{\rightarrow} u(x, y)=u_{0}(x)+\chi(x, y), \quad u_{0} \in H_{0}^{1}(\Omega)^{N}, \quad \chi \in L^{2}\left(\Omega, \mathcal{R}_{1}\right) \\
e\left(u_{\varepsilon, h}(x)\right) \stackrel{2}{\rightarrow} e\left(u_{0}(x)\right)+v(x, y), \quad v \in L^{2}\left(\Omega, \mathcal{V}_{\text {pot }}\right)
\end{gathered}
$$

Далее, если $u_{\varepsilon, h}$ - решения задачи (3.1), то предельная функиия и $(x, y)$ удовлетворяет соотношению (2.6) и, кроме того,

$$
A e\left(u_{\varepsilon, h}(x)\right) \stackrel{2}{\rightarrow} A\left[e\left(u_{0}(x)\right)+v(x, y)\right] \in L^{2}\left(\Omega, \mathcal{V}_{\mathrm{sol}}\right) .
$$

Итак, при любом $h(\varepsilon) \rightarrow 0$ нам довольно много известно о предельной функции $u(x, y)=u_{0}(x)+\chi(x, y)$. Но уравнение, однозначно определяющее функцию $u$, пока не найдено. Усредненное уравнение будет получено отдельно для случая достаточно тонких и для случая достаточно толстых сеток. В первом случае это будет неклассическое уравнение, а во втором - классическое. 


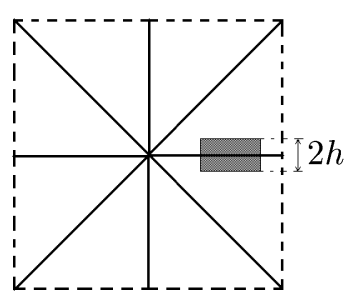

Рис. 11. Носитель вектор-функции $g_{h}$

\section{§ 13. Достаточно тонкие сетки}

Выше показано, что из двух соотношений $(2.6),(2.7)$, определяющих неклассическое усредненное уравнение, первое выполнено при любом $h(\varepsilon) \rightarrow 0$. Покажем, что в случае $h(\varepsilon) \varepsilon^{-1} \rightarrow 0$ выполнено и второе.

Достаточно проверить, что $u-f \perp g$ для $g$ из плотного в $\mathcal{R}_{1}$ множества. В нашем случае $\mathcal{R}_{1}$ - это все нормальные к ребрам векторы из $L_{\mathrm{per}}^{2}(\square, d \mu)^{2}$. Гладкие векторы, финитные около вершин, образуют плотное в $\mathcal{R}_{1}$ множество.

Рассмотрим гладкий вектор $g=\left(0, a\left(y_{1}\right)\right)$, сосредоточенньй внутри отрезка $\left[\delta, \frac{1}{2}-\delta\right] \times\{0\}, \delta>0$. Требуется специальным образом продолжить $g$ на носитель меры $\mu^{h}$. Положим

$$
g_{h}(y)=\left\{-y_{2} a^{\prime}\left(y_{1}\right), a\left(y_{1}\right)\right\} \text { на полосе }\left[\delta, \frac{1}{2}-\delta\right] \times[-h, h]
$$

и $g_{h}(y)=0$ в остальных точках носителя меры $\mu^{h}$ (рис. 11). Тогда

$$
e_{y}\left(g_{h}\right)=\left(\begin{array}{cc}
-y_{2} a^{\prime \prime}\left(y_{1}\right) & 0 \\
0 & 0
\end{array}\right)
$$

на указанной полосе и $e_{y}\left(g_{h}\right)=0$ вне полосы,

$$
\int_{\square}\left|e_{y}\left(g_{h}\right)\right|^{2} d \mu^{h} \leqslant c_{1} h^{2}, \quad \int_{\square}\left|g_{h}-g^{h}\right|^{2} d \mu^{h} \leqslant c_{1} h^{2},
$$

где $g^{h}(y)$ - естественное продолжение исходного вектора $g$. Отсюда

$$
\begin{gathered}
\varepsilon^{-2} \int_{\Omega}\left|e_{y}\left(g_{h}\left(\varepsilon^{-1} x\right)\right)\right|^{2} d \mu_{\varepsilon}^{h} \leqslant c_{2} \varepsilon^{-2} h^{2} \longrightarrow 0 \\
\int_{\Omega}\left|g_{h}\left(\varepsilon^{-1} x\right)-g^{h}\left(\varepsilon^{-1} x\right)\right|^{2} d \mu_{\varepsilon}^{h} \longrightarrow 0
\end{gathered}
$$

Обратимся теперь к тождеству (3.2) и возьмем в нем пробную функцию

$$
\psi(x)=\varphi(x) g_{h}\left(\varepsilon^{-1} x\right), \quad \varphi \in C_{0}^{\infty}(\Omega) .
$$

Тогда получим

$$
\int_{\Omega} A e\left(u_{\varepsilon, h}\right) \cdot \varepsilon^{-1} e_{y}\left(g_{h}\right) \varphi d \mu_{\varepsilon}^{h}+\int_{\Omega} A e\left(u_{\varepsilon, h}\right) \cdot\left[g_{h} \times \nabla \varphi\right] d \mu_{\varepsilon}^{h}=-\int_{\Omega}\left[u_{\varepsilon, h}-f\right] \cdot \varphi g_{h} d \mu_{\varepsilon}^{h} .
$$


Убедимся, что стояшие слева слагаемые сходятся к нулю при $\varepsilon \rightarrow 0$.

Поскольку $A e\left(u_{\varepsilon, h}\right)$ ограничена в $L^{2}\left(\Omega, d \mu_{\varepsilon}^{h}\right)$, первое слагемое сходится к нулю в силу (13.2), а во втором $g_{h}$ можно заменить на $g^{h}$ в силу (13.3). Теперь по двухмасштабной сходимости (12.6) имеем

$\lim _{\varepsilon \rightarrow 0} \int_{\Omega} A e\left(u_{\varepsilon, h}\right) \cdot\left[g_{h} \times \nabla \varphi\right] d \mu_{\varepsilon}^{h}=\int_{\Omega} \int_{\square} A\left[e\left(u_{0}(x)\right)+v(x, y)\right] \cdot[g(y) \times \nabla \varphi(x)] d x d \mu$

с учетом сильной сходимости $g^{h} \longrightarrow g$ в $L^{2}\left(\square, d \mu^{h}\right)$ и леммы 11.5. Интеграл, стоящий справа, равен нулю. Действительно, периодическая матрища $A\left[e\left(u_{0}(x)\right)+\right.$ $v(x, \cdot)]$ соленоидальна (см. (12.6)) и поэтому поточечно ортогональна матрище $g \times$ $\nabla \varphi$.

Итак, левая часть (13.4) имеет нулевой предел. Тогда и

$$
\lim _{\varepsilon \rightarrow 0} \int_{\Omega}\left[u_{\varepsilon, h}(x)-f(x)\right] \cdot \varphi(x) g_{h}\left(\varepsilon^{-1} x\right) d \mu_{\varepsilon}^{h}=0 .
$$

В этом равенстве $g_{h}$ можно заменить на $g^{h}$ (согласно (13.3)), а затем перейти к пределу по двухмасштабной сходимости. В результате получим

$$
\int_{\Omega} \int_{\square}[u(x, y)-f(x)] \cdot \varphi(x) g(y) d x d \mu=0,
$$

и искомое соотношение (2.7) доказано.

Итак, справедлива следуюшая теорема.

Теорема 13.1. Пусть $u_{\varepsilon, h}$ - решение задачи (3.1) и $h(\varepsilon) \varepsilon^{-1} \rightarrow 0$. Тогда имеет место сильная двухмасштабная сходимость

$$
\begin{gathered}
u_{\varepsilon, h}(x) \stackrel{2}{\longrightarrow} u(x, y)=u_{0}(x)+\chi(x, y), \\
e\left(u_{\varepsilon, h}(x)\right) \stackrel{2}{\longrightarrow} e\left(u_{0}(x)\right)+v(x, y),
\end{gathered}
$$

где и - решение неклассического усредненного уравнения (2.10) для предельной меры $\mu$. При әтом $v(x, \cdot)$ - решение периодической задачи (8.4) для $\xi=$ $e\left(u_{0}(x)\right)$.

Стоит отметить, что сильную сходимость (13.6), (13.7) можно переписать в более удобной форме (см. предложение 11.6). Например, соотношение (13.6) означает, что

$$
\lim _{\varepsilon \rightarrow 0} \int_{\Omega}\left|u_{\varepsilon, h}(x)-u_{0}(x)-\chi^{h}\left(x, \varepsilon^{-1} x\right)\right|^{2} d \mu_{\varepsilon}^{h}=0,
$$

где $\chi^{h}$ - естественное продолжение $\chi(x, \cdot)$ на носитель меры $\mu^{h}$.

Аналогично, (13.7) переписывается в виде

$$
\int_{\Omega}\left|e\left(u_{\varepsilon, h}(x)\right)-e\left(u_{0}(x)\right)-v^{h}\left(x, \varepsilon^{-1} x\right)\right|^{2} d \mu_{\varepsilon}^{h}=0,
$$

где

$$
v^{h}(x, \cdot) \longrightarrow v(x, \cdot) \quad \text { в } \quad L^{2}\left(\square, d \mu^{h}\right) .
$$

В частности, $v^{h}(x, y)$ можно определить как решения периодической задачи вида (8.4) для меры $\mu^{h}$. Действительно, свойство (13.8) в этом случае имеет место (см. лемму 16.5). 


\section{§ 14. Достаточно толстые сетки}

14.1. Нужно показать, что в случае $\lim _{\varepsilon \rightarrow 0} h(\varepsilon) \varepsilon^{-1}=\infty$ слабый двухмасштабный предел $u(x, y)$ решений $u_{\varepsilon, h}$ имеет вид $u(x, y)=u_{0}(x)$, и поэтому предельное соотношение (2.6) переходит в классическое усредненное уравнение. Указанные свойства независимости двухмасштабного предела от переменной $y$ в данном случае не связаны с уравнениями, а относятся к произвольной последовательности $u_{\varepsilon, h}$ такой, что $u_{\varepsilon, h}, e\left(u_{\varepsilon, h}\right)$ ограничены в $L^{2}\left(\Omega, d \mu_{\varepsilon}^{h}\right)$.

Докажем равенство

$$
\int_{\square} u(x, y) \cdot a(y) d \mu=0 \quad \forall a \in L^{2}(\square, d \mu)^{2}, \quad \int_{\square} a d \mu=0,
$$

из которого следует, что $u(x, y)$ не зависит от $y, u(x, y)=u_{0}(x)+\chi(x)$. Так как заранее известно, что $\chi \in \mathcal{R}_{1}$, то из единственности разложения (2.1) следует, что $\chi=0$.

Воспользуемся следуюшим утверждением, которое докажем позже.

ЛЕмма 14.1. Для любого вектора $a \in L^{2}\left(\square, d \mu^{h}\right)^{2}, \int_{\square} a d \mu^{h}=0$, найдется симметрическая матрича $b \in L^{2}\left(\square, d \mu^{h}\right)^{3}$ такая, что $\operatorname{div} b=a$ (в смысле мерьи $\left.\mu^{h}\right) u$

$$
\int_{\square}|b|^{2} d \mu^{h} \leqslant \frac{c}{h^{2}} \int_{\square}|a|^{2} d \mu^{h},
$$

где постоянная с не зависит от а и $h$.

Из этой леммы легко получить искомое равенство (14.1). Действительно, возьмем вектор $a \in L^{2}(\square, d \mu)^{2}$ с нулевым средним и естественным образом продолжим его на $h$-сетку. Тогда $\int_{\square} a^{h} d \mu^{h}=0$ и можно определить соответствующую матрицу $b=b^{h}$.

Рассмотрим тождество (12.3). Из оценки (14.2) имеем соотношение

$$
\varepsilon^{2} \int_{\Omega}\left|b^{h}\left(\varepsilon^{-1} x\right)\right|^{2} d \mu_{\varepsilon}^{h} \leqslant c \varepsilon^{2} h^{-2} \rightarrow 0,
$$

поэтому левая часть в (12.3) сходится к нулю при $\varepsilon \rightarrow 0$. В результате получаем равенство

$$
\int_{\Omega} \int_{\square} \varphi(x) a(y) \cdot u(x, y) d x d \mu=0,
$$

из которого вытекает (14.1).

Сама лемма 14.1 есть следствие неравенства Корна (3.6). Действительно, рассмотрим периодическую задачу

$$
v \in \mathcal{H}_{\text {per }}^{1}\left(\square, d \mu^{h}\right), \quad \operatorname{div}(e(v))=a, \quad \int_{\square} a d \mu^{h}=0 .
$$

Из вариационной формулировки

$$
\int_{\square} e(v) \cdot e(\varphi) d \mu^{h}=-\int_{\square} a \cdot \varphi d \mu^{h}, \quad \varphi \in \mathcal{H}_{\mathrm{per}}^{1},
$$


и неравенства Корна (3.6) имеем

$$
\begin{aligned}
\int_{\square} e(v) \cdot e(v) d \mu^{h} & =-\int_{\square} a \cdot v d \mu^{h} \leqslant\left(\int_{\square}|a|^{2} d \mu^{h}\right)^{\frac{1}{2}}\left(\int_{\square}|v|^{2} d \mu^{h}\right)^{\frac{1}{2}} \\
& \leqslant \frac{\sqrt{c}}{h}\left(\int_{\square}|a|^{2} d \mu^{h}\right)^{\frac{1}{2}}\left(\int_{\square} e(v) \cdot e(v) d \mu^{h}\right)^{\frac{1}{2}} .
\end{aligned}
$$

Отсюда получаем

$$
\int_{\square} e(v) \cdot e(v) d \mu^{h} \leqslant \frac{c}{h^{2}} \int_{\square}|a|^{2} d \mu^{h},
$$

и достаточно положить $b=e(v)$.

Итак, справедлив следуюший результат.

Tеорема 14.2. Пусть $u_{\varepsilon, h}$ - решение задачи (3.1) и $h(\varepsilon) \varepsilon^{-1} \rightarrow \infty$. Тогда имеет место сильная сходимость $u_{\varepsilon, h}(x) \longrightarrow u_{0}(x)$ в $L^{2}\left(\Omega, d \mu_{\varepsilon}^{h}\right)$, где $u_{0}(x)$ решение классического усредненного уравнения. Кроме того, имеет место сильная двухмасштабная сходимость (13.7).

14.2. Теперь докажем неравенство Корна (3.6). Рассмотрим одну полосу $I^{h}=$ $[0,1] \times[0, h]$ и проверим, что

$$
\int_{I^{h}}|\nabla u|^{2} d x \leqslant \frac{c}{h^{2}} \int_{I^{h}} e(u) \cdot e(u) d x
$$

для любой гладкой периодической по $x_{1}$ вектор-функции $u(x)=u\left(x_{1}, x_{2}\right)$. Для этого рассмотрим квадрат $I^{1}=[0,1] \times[0,1]$ и воспользуемся классическим неравенством Корна

$$
\int_{I^{1}}|\nabla v|^{2} d y \leqslant c \int_{I^{1}} e(v) \cdot e(v) d y
$$

в котором $v=v\left(y_{1}, y_{2}\right)$ - гладкая периодическая по $y_{1}$ функция (в силу периодичности по $y_{1}$ жесткие перемешения - только константы, и поэтому неравенство выполнено; см. начало $§ 17$ и оценку (17.2)). Делаем замену

$$
x_{1}=y_{1}, \quad x_{2}=h y_{2}, \quad u_{1}=v_{1}, \quad u_{2}=h v_{2} .
$$

Тогда неравенство (14.4) примет вид

$$
\begin{gathered}
\int_{I^{h}}\left[\left(\frac{\partial u^{1}}{\partial x_{1}}\right)^{2}+h^{2}\left(\frac{\partial u^{1}}{\partial x_{2}}\right)^{2}+h^{2}\left(\frac{\partial u^{2}}{\partial x_{1}}\right)^{2}+h^{4}\left(\frac{\partial u^{2}}{\partial x_{2}}\right)^{2}\right] d x \\
\leqslant c \int_{I^{h}}\left[\left(\frac{\partial u^{1}}{\partial x_{1}}\right)^{2}+\frac{h^{2}}{2}\left(\frac{\partial u^{1}}{\partial x_{2}}+\frac{\partial u^{2}}{\partial x_{1}}\right)^{2}+h^{4}\left(\frac{\partial u^{2}}{\partial x_{2}}\right)^{2}\right] d x
\end{gathered}
$$

Из него следует

$$
\int_{I^{h}}\left[\left(\frac{\partial u^{1}}{\partial x_{2}}\right)^{2}+\left(\frac{\partial u^{2}}{\partial x_{1}}\right)^{2}\right] d x \leqslant \frac{c}{h^{2}} \int_{I^{h}} e(u) \cdot e(u) d x .
$$


Поскольку

$$
\int_{I^{h}}\left[\left(\frac{\partial u^{1}}{\partial x_{1}}\right)^{2}+\left(\frac{\partial u^{2}}{\partial x_{2}}\right)^{2}\right] d x \leqslant \int_{I^{h}} e(u) \cdot e(u) d x
$$

то неравенство (14.3) доказано.

Тонкая сетка составлена из четырех полосок рассмотренного выше вида (см. рис. 12). Поэтому из (14.3) получаем оценку

$$
\int_{\square}|\nabla u|^{2} d \mu^{h} \leqslant \frac{4 c}{h^{2}} \int_{\square} e(u) \cdot e(u) d \mu^{h}, \quad u \in C_{\mathrm{per}}^{\infty}(\square)^{N},
$$

которая вместе с неравенством Пуанкаре (3.5) приводит к неравенству Корна (3.6).

Заметим, что это неравенство также справедливо для трехмерных сеток. Доказательство для трехмерного случая технически несколько сложнее, оно приведено в $§ 17$.

14.3. Для полноты изложения докажем неравенство Пуанкаре (3.5). Начнем со специальной оценки для одной полосы $I^{h}=\left[-\frac{1}{2}, \frac{1}{2}\right] \times[-h, h]$. Пусть $B_{h}-$ круг радиуса $h$, принадлежаший полосе $I^{h}$, например $B_{h}=\{|x| \leqslant h\}$ (см. рис. 13). Тогда

$$
\frac{1}{2} \int_{I^{h}}|u|^{2} d x \leqslant 2 \int_{I^{h}}|\nabla u|^{2} d x+\frac{2 h}{\left|B_{h}\right|} \int_{B_{h}}|u|^{2} d x .
$$

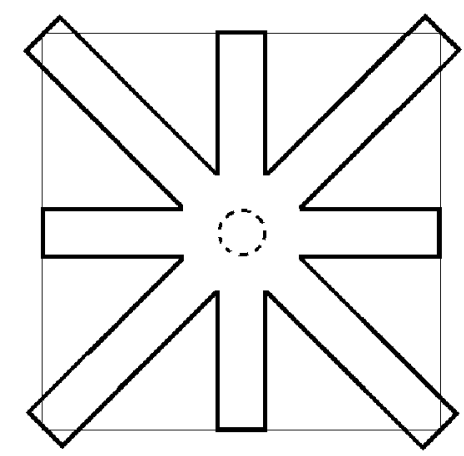

Рис. 12. Тонкая сетка

Точки $x \in I^{h}, x^{\prime} \in B_{h}$ соединим ломаной, как указано на рис. 12 . Имеем

$$
\begin{gathered}
u(x)=\left(u(x)-u\left(x^{\prime}\right)\right)+u\left(x^{\prime}\right) \\
\frac{1}{2} u^{2}(x) \leqslant\left(u(x)-u\left(x^{\prime}\right)\right)^{2}+u^{2}\left(x^{\prime}\right) \leqslant \int_{-\frac{1}{2}}^{\frac{1}{2}}\left|\frac{\partial u}{\partial x_{1}}\left(t_{1}, x_{2}\right)\right|^{2} d t_{1} \\
+h \int_{-h}^{h}\left|\frac{\partial u}{\partial x_{2}}\left(x_{1}^{\prime}, t_{2}\right)\right|^{2} d t_{2}+u^{2}\left(x^{\prime}\right) .
\end{gathered}
$$

Интегрируя это неравенство по $x$ и $x^{\prime}, x \in I^{h}, x^{\prime} \in B_{h}$, получим (14.5). 
Рассмотрим теперь $h$-сетку. При этом нет необходимости считать функции периодическими. Сама $h$-сетка изображена на рис. 13 . Все $h$-стержни содержат круг $B_{h}$ радиуса $h$. Воспользуемся неравенством Пуанкаре для круга:

$$
\int_{B_{h}}|v|^{2} d x \leqslant c h^{2} \int_{B_{h}}|\nabla v|^{2} d x, \quad v \in C^{\infty}\left(B_{h}\right), \quad \int_{B_{h}} v d x=0 .
$$

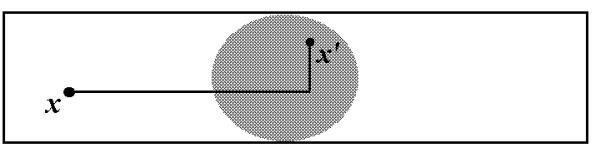

Р ис. 13. Круг $B_{h}$ в доказательстве неравенства Пуанкаре

Рассмотрим функцию

$$
v=u(x)-m, \quad m=\frac{1}{\left|B_{h}\right|} \int_{B_{h}} u d x .
$$

Складьвая неравенства (14.5), примененные к функции $v$, и используя (14.6), получим

$$
\int_{F^{h}}(u-m)^{2} d x \leqslant c \int_{F^{h}}|\nabla u|^{2} d x
$$

где через $F^{h}$ обозначена $h$-сетка. Отсюда следует неравенство Пуанкаре (3.5).

\section{§ 15. О критическом случае в теории тонких сеток}

Покажем, каким не может быть усредненное уравнение в критическом случае.

Было установлено, что как в случае достаточно тонких сеток, так и в случае достаточно толстых сеток решение $u_{\varepsilon, h}$ задачи (3.1) и тензор деформации $e\left(u_{\varepsilon, h}\right)$ обладают свойством сильной двухмасштабной компактности.

В критическом случае это свойство компактности теряет силу.

ПРЕДЛОЖЕНИЕ 15.1. Пусть $u_{\varepsilon, h}-$ решение задачи (3.1) $c h(\varepsilon) \sim \varepsilon$. Тогда последовательности $u_{\varepsilon, h}, e\left(u_{\varepsilon, h}\right)$ не могут быть одновременно компактными в смыслле сильной двухмасштабной сходимости.

ДоКАЗАТЕЛЬСТво. Допустим противное, именно

$$
\begin{gathered}
u_{\varepsilon, h}(x) \stackrel{2}{\longrightarrow} u(x, y)=u_{0}(x)+\chi(x, y), \\
e\left(u_{\varepsilon, h}(x)\right) \stackrel{2}{\longrightarrow} e\left(u_{0}(x)\right)+v(x, y), \quad v \in L^{2}\left(\Omega, \mathcal{V}_{\text {pot }}\right) .
\end{gathered}
$$

Шаг 1. Пусть $a \in L^{2}(\square, d \mu)^{2}, \int_{\square} a d \mu=0, a^{h}$ - естественное продолжение $a$ и $\operatorname{div} b^{h}=a^{h}$ (в смысле меры $\left.\mu^{h}\right)$, где матрица $b^{h}$ задается леммой 14.1.

Перейдем к пределу в тождестве (12.3), используя сильную сходимость (15.1). Предварительно изучим матрицу $\varepsilon b^{h}\left(\varepsilon^{-1} x\right)$. Из оценки (14.2) следует, что она ограничена в $L^{2}\left(\Omega, d \mu_{\varepsilon}^{h}\right)$. Пусть

$$
b_{\varepsilon}(x)=\varepsilon b^{h}\left(\varepsilon^{-1} x\right) \stackrel{2}{\rightarrow} z(x, y) .
$$


Используем тождество

$$
\int_{\Omega} b_{\varepsilon}(x) \cdot e(\psi(x)) d \mu_{\varepsilon}^{h}=-\int_{\Omega} a^{h}\left(\varepsilon^{-1} x\right) \cdot \psi(x) d \mu_{\varepsilon}^{h}, \quad \psi \in C_{0}^{\infty}(\Omega)^{2} .
$$

Так как $a^{h}\left(\varepsilon^{-1} x\right) \stackrel{2}{\rightarrow} a(y)$ (по лемме 11.5$)$, то

$$
\lim _{h \rightarrow 0} \int_{\Omega} a^{h}\left(\varepsilon^{-1} x\right) \cdot \psi(x) d \mu_{\varepsilon}^{h}=\int_{\Omega} \int_{\square} a(y) \cdot \psi(x) d x d \mu=\int_{\square} a d \mu \cdot \int_{\Omega} \psi d x=0,
$$

и поэтому (см. (15.3))

$$
0=\int_{\Omega} \int_{\square} z(x, y) \cdot e(\psi(x)) d x d \mu=\int_{\Omega}\langle z\rangle \cdot e(\psi) d x .
$$

Отсюда $\operatorname{div}_{x}\langle z\rangle=0$. Далее, взяв в тождестве (15.3) пробную функцию $\psi=$ $\varepsilon \varphi(x) w\left(\varepsilon^{-1} x\right)$, где $\varphi \in C_{0}^{\infty}(\Omega), w \in C_{\mathrm{per}}^{\infty}(\square)^{N}$, получим $\operatorname{div}_{y} z=0$. Итак,

$$
\operatorname{div}_{y} z=0, \quad \operatorname{div}_{x}\langle z\rangle=0 .
$$

Теперь можно перейти к пределу в тождестве (12.3), используя сильную двухмасштабную сходимость (15.1), (15.2). В результате получим

$$
\int_{\Omega} \int_{\square} \varphi\left(e\left(u_{0}\right)+v\right) \cdot z d x d \mu+\int_{\Omega} \int_{\square}(u \times \nabla \varphi) \cdot z d x d \mu=-\int_{\Omega} \int_{\square} \varphi a \cdot u d x d \mu .
$$

Оба слагаемые в левой части можно упростить. Действительно, так как $v \in$ $L^{2}\left(\Omega, \mathcal{V}_{\text {pot }}\right)$, a $z \in L^{2}\left(\Omega, \mathcal{V}_{\text {sol }}\right)$, то (см. ортогональное разложение $\left.(9.11)\right)$

$$
\int_{\Omega} \int_{\square} \varphi\left(e\left(u_{0}\right)+v\right) \cdot z d x d \mu=\int_{\Omega} e\left(u_{0}\right) \cdot\langle z\rangle d x .
$$

Далее, вектор $z$ тангенщиален, и поэтому $(\chi \times \nabla \varphi) \cdot z=0$ (лемма 5.3). Следовательно, второе слагаемое, стоящее слева в (15.4), равно

$$
\int_{\Omega} \int_{\square}\left(u_{0} \times \nabla \varphi\right) \cdot z d x d \mu=\int_{\Omega}\left(u_{0} \times \nabla \varphi\right) \cdot\langle z\rangle d x .
$$

В силу равенства $\operatorname{div}_{x}\langle z\rangle=0$ сумма величин (15.5) и (15.6) равна нулю. Тогда и правая часть в (15.4) равна нулю. Получили равенство (14.1), из которого следует, что $\chi=0$. Итак, предельное уравнение обязано быть классическим. Отметим, что само уравнение (3.1) не было использовано. Фактически доказано, что если $u_{\varepsilon, h}$ - произвольная последовательность такая, что $u_{\varepsilon, h}, e\left(u_{\varepsilon, h}\right)$ компактны в смысле сильной двухмасштабной сходимости, то предел $u_{\varepsilon, h}$ не зависит от $y$.

Шаг 2. Повторим рассуждения из $\S 13$, связанные с аппроксимацией гладкого вектора $g \in \mathcal{R}_{1}$. В нашем случае последовательность $\varepsilon^{-1} e_{y}\left(g_{h}\left(\varepsilon^{-1} x\right)\right)$ ограничена в $L^{2}\left(\Omega, d \mu_{\varepsilon}^{h}\right)$, но не сходится к нулю сильно, как в $\S 13$. Из явной формулы для $e_{y}\left(g_{h}\right)$ (см. (13.1)) нетрудно заключить, что

$$
\varepsilon^{-1} e_{y}\left(g_{h}\left(\varepsilon^{-1} x\right)\right) \stackrel{2}{\rightarrow} 0 .
$$


Теперь рассмотрим тождество (13.4). Первое слагаемое в левой части этого тождества сходится к нулю в силу (15.7) и (15.1) (см. определение 11.3). Второе также сходится к нулю - это устанавливается тем же рассуждением из $\S 13$. В результате получаем равенство (13.5), из которого следует, что предельное уравнение является неклассическим (при этом мы использовали только компактность последовательности $\left.e\left(u_{\varepsilon, h}\right)\right)$. Полученное противоречие доказывает наше утверждение.

Сильная сходимость (15.1) эквивалентна двум свойствам (10.3) и (10.4). Вывод этих свойств в $\S 10$ сушественно использовал энергетическое равенство (3.4). Отсюда следует, что энергетическое равенство, справедливое в обоих некритических случаях, в критическом случае не выполняется.

\section{$\S 16$. О других тонких структурах}

16.1. Допустим, что $\mu^{h}, \mu$ - произвольные периодические борелевы меры, $\mu^{h} \rightarrow \mu$. Сформулируем в общем виде аппроксимативные свойства мер $\mu^{h}$, обеспечивающие справедливость теорем, аналогичных теоремам 9.4 и 9.5.

Пусть $\mathcal{R}$ - множество периодических жестких перемещений, отвечающих предельной мере $\mu, \mathcal{A}^{\text {hom }}$ - усредненный тензор, также соответствующий предельной мере $\mu$. Предполагается, что для $\mathcal{R}$ выполнено основное разложение (9.10) и тензор $\mathcal{A}^{\text {hom }}$ невырожден.

Аппроксимативные свойства следуюшие:

(i) для любого вектора $a$ из некоторого плотного в $\mathcal{R}^{\perp} \subset L^{2}(\square, d \mu)^{N}$ множества найдутся вектор $a^{h} \in L^{2}\left(\square, d \mu^{h}\right)^{N}$ и матрища $b^{h} \in L^{2}\left(\square, d \mu^{h}\right)^{\frac{N(N+1)}{2}}$ такие, что $a^{h}=\operatorname{div} b^{h}$ (в смысле меры $\left.\mu^{h}\right), a^{h} \longrightarrow a, b^{h} \longrightarrow b$ (вместо сильной сходимости $b^{h} \longrightarrow b$ часто достаточно просто ограниченности $b^{h}$ в $\left.L^{2}\left(\square, d \mu^{h}\right)\right)$;

(ii) для любой матрищы $b \in \mathcal{V}_{\text {sol }}(\square, d \mu)$ найдется матрища $b^{h} \in \mathcal{V}_{\text {sol }}\left(\square, d \mu^{h}\right)$ такая, что $b^{h} \longrightarrow b$ в $L^{2}\left(\square, d \mu^{h}\right)$ (сильная аппроксимируемость соленоидальных матриц).

Свойство (i) обеспечивает доказательство аналога теоремы 9.4, а свойство (ii) аналога основной теоремы 9.5. Лемма 9.6 о сходимости моментов является следствием теоремы 9.5.

Теперь обратимся к задаче (3.1) и теореме 10.1. Смысл этой теоремы заключается в выводе двух предельных соотношений (10.1) и (10.2). Шаг 1, в результате которого получено соотношение (10.1), можно легко повторить, поскольку в нем использована только лемма о сходимости моментов. Таким образом, соотношение (10.1), совпадаюшее в данном случае с (2.6), остается в силе. Получаем следующее утверждение.

ТЕОРЕМа 16.1. Пусть $h(\varepsilon) \rightarrow 0$ произвольно $и u_{\varepsilon, h}$ - последовательность из $\mathcal{H}_{0}^{1}\left(\Omega, d \mu_{\varepsilon}^{h}\right)$ такая, что $u_{\varepsilon, h}, e\left(u_{\varepsilon, h}\right)$ ограничены в $L^{2}\left(\Omega, d \mu_{\varepsilon}^{h}\right)$. Тогда ( точностью до выделения подпоследовательности) имеет место двухмаситабная сходимость (12.4), (12.5).

Далее, если $u_{\varepsilon, h}$ - решения задачи (3.1), то предельная функция $и(x, y)$ удовлетворяет соотношению (2.6), а также имеет место сходимость (12.6).

Можно показать, что свойства (i), (ii) выполнены не только для тонких сеток 
(как это было проверено ранее), но и для соответствуюших аппроксимаций составной структуры и ящичной структуры (см. рис. 14).

Проверим свойство (i) для тонкой ящичной структуры.

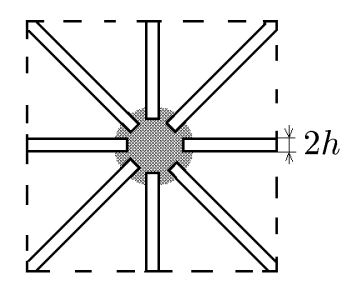

Тонкая составная структура

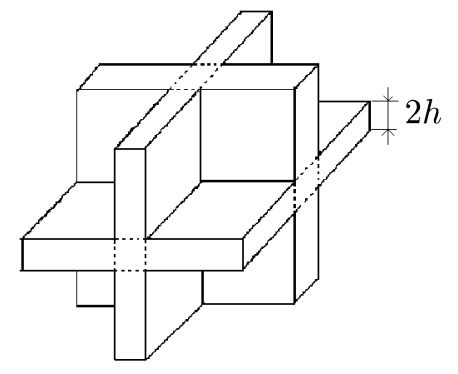

Тонкая ящичная структура

Рис. 14

Рассмотрим вектор $a \in L^{2}(\square, d \mu), a \perp \mathcal{R}$. Из $a \perp \mathcal{R}$ следует, что на каждой грани вектор а параллелен этой грани и имеет нулевое среднее по грани. На каждой из трех плоскостей периодическая задача

$$
\operatorname{div} e(z)=a
$$

(в смысле соответствующей плоской меры) имеет решение. Возьмем сумму этих решений и через $a^{h}$ и $b^{h}$ обозначим естественное продолжение вектора $a$ и матрищы $e(z)$. Тогда $\operatorname{div} b^{h}=a^{h}$ в смысле меры $\mu^{h}$. Свойство (i) доказано.

Свойство (ii) для ящичной структуры доказывается несколько сложнее. Пространство $\mathcal{V}_{\text {sol }}(\square, d \mu)$ не расщепляется (оно шире, чем это следовало бы из принципа расщепления). Тем не менее свойство (ii) справедливо как для тонкой ящичной структуры, так и для тонкой составной структуры. Ниже (см. теорему 16.2) оно будет доказано в общем случае, когда $\mu^{h}$ - сглаживание произвольной меры.

До сих пор речь шла о тонких структурах (составной и ящичной), но сама толшина $h=h(\varepsilon) \rightarrow 0$ была произвольной. Было получено свойство (2.6), которое не определяет однозначно саму вектор-функцию $u(x, y)$. Как и в случае тонких сеток, возникают два варианта:

1) очень тонкие структуры: $\lim _{\varepsilon \rightarrow 0} h(\varepsilon) \varepsilon^{-1}=0$;

2) не очень тонкие структуры: $\lim _{\varepsilon \rightarrow 0} h(\varepsilon) \varepsilon^{-1}=\infty$.

В первом случае нетрудно повторить рассуждения из $\S 13$, связанные со специальной аппроксимацией периодических жестких перемещений, и к свойству (2.6) добавить (2.7). Иначе говоря, в этом случае справедлив неклассический принцип усреднения.

Во втором случае действует классическое усреднение. Для этого достаточно доказать неравенство Корна типа (3.6) и вывести из него независимость двухмасштабного предела решений от переменной $y$.

16.2. Укажем пример общего характера, в котором выполнены аппроксимативные свойства. 
ТЕОРема 16.2. Пусть $\mu$ - произвольная периодическая борелева мера, $\mu^{h}-$ ее сглаживание (см. (3.7)). Тогда аппроксимативные свойства (i), (ii) имеют место.

ДокАЗАТЕльСтво. По функции $b \in L^{2}(\square, d \mu)$ определим $b^{h} \in L^{2}\left(\square, d \mu^{h}\right)$ равенством

$$
\int_{\square} b^{h} \varphi d \mu^{h}=\int_{\square} b(\varphi)_{h} d \mu, \quad \varphi \in C_{\mathrm{per}}(\square),
$$

где $\mu^{h}$ и $(\varphi)_{h}$ определены в $(3.7),(3.8)$.

Для доказательства существования такой функции $b^{h}$ рассмотрим очевидную оценку

$$
\left(\int_{\square} b(\varphi)_{h} d \mu\right)^{2} \leqslant C \int_{\square}\left((\varphi)_{h}\right)^{2} d \mu, \quad C=\int_{\square} b^{2} d \mu .
$$

По интегральному неравенству Йенсена имеем $\left((\varphi)_{h}\right)^{2} \leqslant\left(\varphi^{2}\right)_{h}$, поэтому

$$
\int_{\square}\left((\varphi)_{h}\right)^{2} d \mu \leqslant \int_{\square}\left(\varphi^{2}\right)_{h} d \mu=\int_{\square} \varphi^{2} d \mu^{h}
$$

в силу определения меры $\mu^{h}$ (см. (3.7)). Следовательно, функция $b^{h}$ сушествует по теореме Рисса о представлении и справедливо неравенство

$$
\int_{\square}\left(b^{h}\right)^{2} d \mu^{h} \leqslant \int_{\square} b^{2} d \mu .
$$

Далее, так как $(\varphi)_{h} \rightarrow \varphi$ равномерно, то из определения (16.1) получим слабую сходимость $b^{h} \rightarrow b$ в $L^{2}\left(\square, d \mu^{h}\right)$. Но тогда оценка (16.2) показывает, что эта сходимость является сильной: $b^{h} \longrightarrow b$ в $L^{2}\left(\square, d \mu^{h}\right)$ (см. свойство полунепрерывности (11.6) и предложение 11.4).

После этого рассмотрим матрицу $b \in \mathcal{V}_{\text {sol }}(\square, d \mu)$ и заметим, что соответствующая ей матрица $b^{h}$ будет соленоидальной относительно меры $\mu^{h}$. Действительно, для $\varphi=e(\psi), \psi \in C_{\mathrm{per}}^{\infty}(\square)^{N}$, из равенства (16.1) получим

$$
\int_{\square} b^{h} \cdot \varphi d \mu^{h}=\int_{\square} b \cdot(\varphi)_{h} d \mu=0,
$$

так как $(\varphi)_{h}=e\left((\psi)_{h}\right)$. Итак, проверено, что $b^{h} \longrightarrow b$ в $\mathcal{V}_{\text {sol }}\left(\square, d \mu^{h}\right)$. Аппроксимативное свойство (ii) доказано.

Проверим аппроксимативное свойство (i).

Пусть $\mathcal{R}$ - множество периодических жестких перемешений, отвечающих мере $\mu$. Согласно лемме 9.3 векторы $a$ из плотного в $\mathcal{R}^{\perp}$ множества допускают представление в виде дивергенции, $\operatorname{div} b=a$ (в смысле меры $\mu$ ).

Для соответствующей матрицы $b^{h}$ по определению имеем (см. (16.1))

$$
\begin{aligned}
\int_{\square} b^{h} \cdot e(\psi) d \mu^{h} & =\int_{\square} b \cdot e\left((\psi)_{h}\right) d \mu=-\int_{\square} a \cdot(\psi)_{h} d \mu \\
& =-\int_{\square} a^{h} \cdot \psi d \mu^{h}, \quad \psi \in C_{\mathrm{per}}^{\infty}(\square) .
\end{aligned}
$$

Получили соотношение $\operatorname{div} b^{h}=a^{h}$ (в смысле меры $\mu^{h}$ ), причем по доказанному ранее $b^{h} \longrightarrow b, a^{h} \longrightarrow a$ в $L^{2}\left(\square, d \mu^{h}\right)$. Тем самым проверено аппроксимативное свойство (i). Теорема доказана. 
СлЕДСТВИЕ 16.3. Предположим, что:

1) $\mu$ - произвольная периодическая борелева мера, $\mu^{h}-$ ее сглаживание, $h(\varepsilon) \rightarrow 0$ произвольно;

2) для периодических жестких перемещений, отвечающих мере $\mu$, выполнено разложение (9.10);

3) усредненный тензор, отвечающий мере $\mu$, невырожден (это условие приводится только для простоты формулировок).

Тогда справедливы все утверждения теоремы 16.1.

16.3. Рассмотрим некоторые вопросы, связанные со свойством (ii) п. 16.1 сильной аппроксимируемости соленоидальных матриц.

Пусть $v^{h} \in \mathcal{V}_{\text {pot }}\left(\square, d \mu^{h}\right)$ и $v^{h} \rightarrow v$ в $L^{2}\left(\square, d \mu^{h}\right)$. Будет ли $v$ принадлежать $\mathcal{V}_{\text {pot }}(\square, d \mu)$ ? Другими словами, сохранится ли потенщиальность при слабой сходимости?

Лемма 16.4. Свойство сохранения потенциальности матрии при слабой сходимости эквивалентно свойству сильной аппроксимируемости соленоидальных матрич.

ДокаЗАТЕЛЬСтво. Пусть выполнено свойство (ii) п. 16.1. Рассмотрим равенство

$$
\int_{\square} v^{h} \cdot b^{h} d \mu^{h}=0
$$

в котором $b^{h}$ - соленоидальная матрица из (ii). Отсюда (см. разложение (8.1))

$$
\int_{\square} v \cdot b d \mu=0 \quad \forall b \in \mathcal{V}_{\mathrm{sol}}(\square, d \mu) \Longrightarrow v \in \mathcal{V}_{\mathrm{pot}}(\square, d \mu) .
$$

Докажем обратное. Для $b \in \mathcal{V}_{\mathrm{sol}}(\square, d \mu)$ возьмем какую-нибудь последовательность

$$
\tilde{b}^{h} \longrightarrow b \text { в } L^{2}\left(\square, d \mu^{h}\right)
$$

и рассмотрим задачу $u^{h} \in \mathcal{H}_{\mathrm{per}}^{1}\left(\square, d \mu^{h}\right), \operatorname{div} e\left(u^{h}\right)=\operatorname{div} \tilde{b}^{h}$ (в смысле меры $\left.\mu^{h}\right)$. Из тождества

$$
\int_{\square} e\left(u^{h}\right) \cdot e(\varphi) d \mu^{h}=\int_{\square} \tilde{b}^{h} \cdot e(\varphi) d \mu^{h} \quad \forall \varphi \in C_{\mathrm{per}}^{\infty}(\square)^{N}
$$

следует, что $e\left(u^{h}\right)$ ограничена в $L^{2}\left(\square, d \mu^{h}\right)$. Если $e\left(u^{h}\right) \rightarrow z$ в $L^{2}\left(\square, d \mu^{h}\right)$, то

$$
\int_{\square} z \cdot e(\varphi) d \mu=\int_{\square} b \cdot e(\varphi) d \mu=0,
$$

т.е. $z \in \mathcal{V}_{\text {sol }}(\square, d \mu)$. С другой стороны, $z \in \mathcal{V}_{\text {pot }}(\square, d \mu)$; отсюда $z=0$, и поэтому (cм. (16.3))

$$
\lim _{h \rightarrow 0} \int_{\square} e\left(u^{h}\right) \cdot e\left(u^{h}\right) d \mu^{h}=\lim _{h \rightarrow 0} \int_{\square} \tilde{b}^{h} \cdot e\left(u^{h}\right) d \mu^{h}=\int_{\square} b \cdot z d \mu=0 .
$$

Остается положить $b^{h}=\tilde{b}^{h}-e\left(u^{h}\right)$, чтобы получить (ii). Лемма доказана. 
Между потенщиальными и соленоидальными матрищами имеется существенное различие. Так, слабый предел соленоидальных матриц - всегда соленоидальная матрица, что ясно из определения соленоидальной матрицы. Для этого ничего не требуется, кроме $\mu^{h} \rightarrow \mu$. С другой стороны, для последовательности $\mu^{h} \rightarrow \mu$ общего вида свойство сильной аппроксимируемости (ii) может не вьполняться, а слабый предел потенциальных матриц - уже необязательно потенциальная матрица. Подробное обсуждение этих вопросов, однако, выходит за рамки настоящей работы.

Укажем некоторые применения аппроксимативных свойств.

ЛЕмма 16.5. Пусть выполняется свойство (ii) сильной аппроксимируемости соленоидальных матрич. Тогда

$$
\lim _{h \rightarrow 0} A_{h}^{\text {hom }}=A^{\text {hom }},
$$

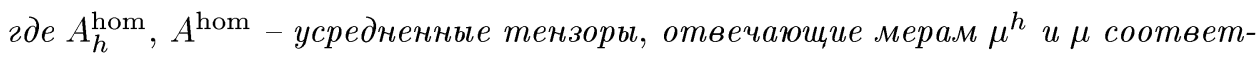
ственно.

При этом имеет место сильная в $L^{2}\left(\square, d \mu^{h}\right)$ сходимость решений периодической задачи вида (8.4) для меры $\mu^{h} к$ решению такой же задачи для меры $\mu$.

ДокАЗАТЕЛЬСтво. Рассмотрим периодическую задачу вида (8.4) для меры $\mu^{h}$, т.e.

$$
v^{h} \in \mathcal{V}_{\mathrm{pot}}\left(\square, d \mu^{h}\right), \quad \int_{\square} A\left(\xi+v^{h}\right) \cdot e(\varphi) d \mu^{h}=0, \quad \varphi \in C_{\mathrm{per}}^{\infty}(\square)^{N} .
$$

Легко проверяется, что последовательность $v^{h}$ ограничена в $L^{2}\left(\square, d \mu^{h}\right)$, и поэтому можно считать, что она слабо сходится: $v^{h} \rightarrow v$. По доказанному $v \in$ $\mathcal{V}_{\text {pot }}(\square, d \mu)$, и переходом к пределу получим

$$
v \in \mathcal{V}_{\mathrm{pot}}(\square, d \mu), \quad \int_{\square} A(\xi+v) \cdot e(\varphi) d \mu=0, \quad \varphi \in C_{\mathrm{per}}^{\infty}(\square)^{N} .
$$

Итак, $v$ - решение уравнения (8.4). Отсюда

$$
A_{h}^{\text {hom }} \xi \cdot \xi=\int_{\square} A\left(\xi+v^{h}\right) \cdot \xi d \mu^{h} \longrightarrow \int_{\square} A(\xi+v) \cdot \xi d \mu=A^{\text {hom }} \xi \cdot \xi .
$$

Ясно, что имеет место сильная сходимость $v^{h} \longrightarrow v$. Лемма доказана.

Можно показать, что в общем случае равенство (16.4) не выполняется.

Укажем другое применение аппроксимативных свойств.

Предположим, что $\mu^{h} \rightarrow \mu$ и выполнены аппроксимативные свойства (i), (ii) из п. 16.1 .

ТЕОРема 16.6. Пусть $u_{h} \in \mathcal{H}_{\mathrm{per}}^{1}\left(\square, d \mu^{h}\right)$ и последовательности $u_{h}, e\left(u_{h}\right)$ ограничены в $L^{2}\left(\square, d \mu^{h}\right)$. Если для предельной меры $\mu$ выполнено неравенство Корна (8.7), то (с точностью до выделения подпоследовательности) имеем

$$
\begin{gathered}
u_{h} \rightarrow u \quad \text { e } \quad L^{2}\left(\square, d \mu^{h}\right), \quad u \in \mathcal{H}^{1}(\square, d \mu), \\
e\left(u_{h}\right) \rightarrow e(u) \quad \text { в } \quad L^{2}\left(\square, d \mu^{h}\right) .
\end{gathered}
$$


ДоКАЗАТЕЛЬСТВО. По лемме 16.4 слабый предел $e\left(u_{h}\right)$ принадлежит пространству $\mathcal{V}_{\text {pot }}(\square, d \mu)$. В силу неравенства Корна (8.7) имеет место представление (8.8), и поэтому $e\left(u_{h}\right) \rightarrow e(v), v \in \mathcal{H}_{\text {per }}^{1}(\square, d \mu)$. Остается сравнить элемент $v$ со слабым пределом $u_{h}$, обозначаемым через $u$. Для этого рассмотрим равенство

$$
-\int_{\square} e\left(u_{h}\right) \cdot b^{h} d \mu^{h}=\int_{\square} a^{h} \cdot u_{h} d \mu^{h},
$$

где $\operatorname{div} b^{h}=a^{h}$, матрица $b^{h}$ и вектор $a^{h}$ такие, как в аппроксимативном свойстве (i). Тогда

$$
-\int_{\square} e(v) \cdot b d \mu=\int_{\square} u \cdot a d \mu,
$$

при этом $\operatorname{div} b=a$ в смысле меры $\mu$. Поэтому

$$
-\int_{\square} e(v) \cdot b d \mu=\int_{\square} v \cdot a d \mu .
$$

Получим, что

$$
\int_{\square}(u-v) \cdot a d \mu=0
$$

для любого $a$ из плотного в $\mathcal{R}^{\perp}$ множества. Отсюда $u-v \in \mathcal{R}$, и теорема доказана.

16.4. Рассмотрим скалярные задачи.

Сформулируем аппроксимативные свойства мер $\mu^{h}$. Напомним, что соотношение $\operatorname{div} b=a$, где $a \in L^{2}(\square, d \mu), b \in L^{2}(\square, d \mu)^{N}$, означает выполнение тождества

$$
\int_{\square} b \cdot \nabla \varphi d \mu=-\int_{\square} a \varphi d \mu, \quad \varphi \in C_{\mathrm{per}}^{\infty} .
$$

Пусть $\mathcal{V}_{\text {sol }}(\square, d \mu)$ - множество соленоидальных векторов.

(i) Для любой функции $a$ из некоторого плотного в $L^{2}(\square, d \mu) / \mathbb{R}^{1}$ множества найдутся векторы $a^{h} \in L^{2}\left(\square, d \mu^{h}\right)$ и $b^{h} \in L^{2}\left(\square, d \mu^{h}\right)^{N}$ такие, что $a^{h}=\operatorname{div} b^{h}$ (в смысле меры $\mu^{h}$ ), $a^{h} \longrightarrow a, b^{h} \longrightarrow b$ (вместо сильной сходимости $b^{h} \longrightarrow b$ часто достаточно ограниченности $b^{h}$ в $\left.L^{2}\left(\square, d \mu^{h}\right)\right)$.

(ii) Для любого вектора $b \in \mathcal{V}_{\text {sol }}(\square, d \mu)$ найдется вектор $b^{h} \in \mathcal{V}_{\text {sol }}\left(\square, d \mu^{h}\right)$ такой, что $b^{h} \longrightarrow b$ в $L^{2}\left(\square, d \mu^{h}\right)$ (сильная аппроксимируемость соленоидальных векторов).

Свойства (i), (ii) обеспечивают классическое усреднение для задачи вида (3.1) при любом $h(\varepsilon) \rightarrow 0$, и в этом состоит основное отличие случая скалярных задач от случая задач теории упругости.

Приведем точную формулировку теоремы усреднения.

Рассмотрим задачу Дирихле

$$
-\operatorname{div} A \nabla u_{\varepsilon, h}+u_{\varepsilon, h}=f_{\varepsilon, h},
$$

где $A$ - постоянная симметрическая матрища, $A>0$. По определению

$$
\begin{gathered}
u_{\varepsilon, h} \in H_{0}^{1}\left(\Omega, d \mu_{\varepsilon, h}\right), \\
\int_{\Omega}\left[A \nabla u_{\varepsilon, h} \cdot \nabla \varphi+u_{\varepsilon, h} \varphi\right] d \mu_{\varepsilon, h}=\int_{\Omega} f_{\varepsilon, h} \varphi d \mu_{\varepsilon}^{h}, \quad \varphi \in C_{0}^{\infty}(\Omega) .
\end{gathered}
$$


Введем задачу на ячейке

$$
v \in \mathcal{V}_{\text {pot }}, \quad \operatorname{div} A(\xi+v)=0
$$

где $\mathcal{V}_{\text {pot }}$ - замыкание множества $\left\{\nabla \varphi, \varphi \in C_{\text {per }}^{\infty}(\square)\right\}$ в $L^{2}(\square, d \mu)^{N}$, и определим усредненную матрицу $A^{\text {hom }}$ равенством $A^{\text {hom }} \xi=\langle A(\xi+v)\rangle$. Для упрошения формулировки считаем, что $A^{\text {hom }}>0$.

ТЕОРема 16.7. Предположим, что:

(a) выполнены аппроксимативные свойства (i), (ii);

(b) $h(\varepsilon) \rightarrow 0$ произвольно, $u_{\varepsilon, h}$ - решение задачи (16.5);

(c) $f_{\varepsilon, h} \longrightarrow f$ сильно в $L^{2}\left(\Omega, d \mu_{\varepsilon}^{h}\right)$.

Тогда $u_{\varepsilon, h}(x) \longrightarrow u(x)$ сильно в $L^{2}\left(\Omega, d \mu_{\varepsilon}^{h}\right)$, где $и(x)$ - решение усредненной задачи

$$
u \in H_{0}^{1}(\Omega), \quad-\operatorname{div} A^{\mathrm{hom}} \nabla u+u=f .
$$

Кроме того, имеет место сильная двухмасштабная сходимость

$$
\nabla u_{\varepsilon, h}(x) \stackrel{2}{\longrightarrow} \nabla u(x)+v(x, y)
$$

где $v(x, \cdot)$ - решение вспомогательной периодической задачи (16.6) для $\xi=\nabla u$.

Нет необходимости останавливаться на доказательстве этой теоремы, а также на различных ее уточнениях, поскольку эти доказательства аналогичны случаю задач теории упругости (и много проше). Важно знать, когда выполнены аппроксимативные свойства (i), (ii).

Если $\mu^{h}-$ сглаживание произвольной әргодической борелевой меры, то оба свойства выполнены.

\section{$\S 17$. Неравенство Корна для трехмерных стержневых структур}

Докажем неравенство Корна (3.6) для трехмерного аналога тонких периодических сеток. Сначала напомним некоторые факты, касающиеся классического неравенства Корна.

Если $\Omega$ - ограниченная липшищева область в $\mathbb{R}^{N}$, то справедливо неравенство

$$
\int_{\Omega} \nabla v \cdot \nabla v d x \leqslant c \int_{\Omega}(v \cdot v+e(v) \cdot e(v)) d x, \quad v \in C^{\infty}(\bar{\Omega})^{N}
$$

в котором $c=c(\Omega)$. Из этого общего неравенства Корна нетрудно получить другие формулировки, среди которых наиболее часто используется следующая (cм. [2], [3]).

Пусть $V$ - подпространство в $H^{1}(\Omega)^{N}$, не содержащее ненулевых жестких перемешений. Тогда

$$
\int_{\Omega}(v \cdot v+\nabla v \cdot \nabla v) d x \leqslant c \int_{\Omega} e(v) \cdot e(v) d x, \quad v \in V,
$$

где $c=c(\Omega, V)$. Напомним, что вектор $v \in H^{1}(\Omega)^{N}$ - это жесткое перемещение, если $e(v)=0$ (в смысле распределений). Известно, что жесткие перемешения 
имеют вид $v=c+A x$, где $c$ - постоянный вектор, а $A$-кососимметрическая матрища. В частности, на плоскости жесткие перемешения определяются формулой $c+t\left(-x_{2}, x_{1}\right)$.

17.1. Установим следуюший результат, относящийся к тонкому круговому цилиндру.

Пусть цилиндр $I^{h}=\left[-\frac{1}{2}, \frac{1}{2}\right] \times Q_{h}$, где $Q_{h}-$ круг радиуса $h$, расположен вдоль оси $x_{1}$. Рассмотрим гладкие периодические по $x_{1}$ вектор-функции $u=\left(u^{1}, u^{2}, u^{3}\right)$. В этом случае жесткие перемещения имеют вид $c+t\left(0,-x_{3}, x_{2}\right)$.

Пусть $B_{h}$ - шар радиуса $h$, содержашийся в данном цилиндре $I^{h}$, например $B_{h}=\{|x|<h\}$.

ЛЕмма 17.1. Справедлива оценка

$$
h^{2} \int_{I^{h}} u \cdot u d x \leqslant c \int_{I^{h}} e(u) \cdot e(u) d x, \quad u \in C_{\mathrm{per}}^{\infty}(\square)^{3},
$$

при условии ортогональности жсестким перемещениям:

$$
0=\int_{B_{h}} u d x=\int_{B_{h}}\left(u^{2} x_{3}-u^{3} x_{2}\right) d x .
$$

Кроме того, для продольной компоненты $u^{1}$ выполнено

$$
\int_{I^{h}}\left(u^{1}\right)^{2} d x \leqslant c \int_{I^{h}} e(u) \cdot e(u) d x, \quad \text { как только } \int_{B_{h}} u^{1} d x=0 .
$$

Эту лемму докажем позже.

Из оценки (17.3) следует неравенство

$$
h^{2} \int_{I^{h}}(u-p) \cdot(u-p) d x \leqslant c \int_{I^{h}} e(u) \cdot e(u) d x, \quad \int_{B_{h}} u d x=0
$$

где $p$ - проекция вектора $u$ на одномерное подпространство $t\left(0,-x_{3}, x_{2}\right)$ в $L^{2}\left(B_{h}\right)^{3}$. Простыми вычислениями получим

$$
\int_{I^{h}} p \cdot p d x \leqslant \frac{c}{h} \int_{B_{h}} u \cdot u d x
$$

В результате

$$
h^{2} \int_{I^{h}} u \cdot u d x \leqslant 2 c\left(h \int_{B_{h}} u \cdot u d x+\int_{I^{h}} e(u) \cdot e(u) d x\right), \quad \int_{B_{h}} u d x=0
$$

Теперь рассмотрим периодические стержневые структуры определенного вида. Предположим, что в кубе периодичности $\left[-\frac{1}{2}, \frac{1}{2}\right]^{3}=\square$ такая структура устроена аналогично тонкой сетке (см. рис. 12), т.е. существует конечное число круговых цилиндров с одинаковым поперечным сечением радиуса $h$, и эти цилиндры содержат общий шар $B_{h}$. При этом число цилиндров должно быть не менее трех. Например, можно взять три цилиндра, идущих вдоль координатных осей, затем 
добавить к ним цилиндры, идушие вдоль диагоналей куба $\square$, и т.д. Возможно рассмотрение и более сложных структур, но сейчас это мы обсуждать не будем.

Оценка (17.5) справедива для стержневой структуры, если только $B_{h}$ - упомянутый выше обший шар. Требуется оценить слагаемое $h \int_{B_{h}} u \cdot u d x$.

По условию шар $B_{h}$ принадлежит, по крайней мере, трем стержням. Следовательно, вектор $\left.u\right|_{B_{h}}$ является линейной комбинацией продольных компонент для некоторых стержней. Для продольных компонент имеются более точные оценки вида (17.4), из которых следует

$$
h \int_{B_{h}} u \cdot u d x \leqslant c_{0} \int_{F^{h}} e(u) \cdot e(u) d x
$$

где через $F^{h}$ обозначена стержневая структура. Отсюда получим оценку

$$
h^{2} \int_{F^{h}} u \cdot u d x \leqslant c \int_{F^{h}} e(u) \cdot e(u) d x, \quad u \in C_{\mathrm{per}}^{\infty}(\square)^{3}, \quad \int_{B_{h}} u d x=0,
$$

из которой следует искомое неравенство Корна (3.6).

17.2. ДоКАЗАТЕЛЬСТво ЛЕмМЫ 17.1. Произведем замену переменных

$$
x_{1}=y_{1}, \quad x_{2}=h y_{2}, \quad x_{3}=h y_{3} .
$$

Тогда тонкий цилиндр $I^{h}$ перейдет в стандартный цилиндр $I^{1}=\left[-\frac{1}{2}, \frac{1}{2}\right] \times Q_{1}$, где $Q_{1}$ - единичньй круг, а шар $B_{h}$ перейдет в эллипсоид $K_{h}=\left\{y_{1}^{2}+h^{2} y_{2}^{2}+\right.$ $\left.h^{2} y_{3}^{2} \leqslant h^{2}\right\}$.

Достаточно проверить, что выполнено неравенство

$$
\int_{I^{1}} v \cdot v d y \leqslant c \int_{I^{1}} e(v) \cdot e(v) d y
$$

при условии периодичности по $y_{1}$ и ортогональности жестким перемешениям, т.е.

$$
0=\int_{K_{h}} v d y=\int_{K_{h}}\left(v^{2} y_{3}-v^{3} y_{2}\right) d y
$$

Действительно, если вернуться к переменным $x$, произведя при этом замену $u^{1}=v^{1}, u^{2}=h^{-1} v^{2}, u^{3}=h^{-1} v^{3}$, то неравенство (17.6) перейдет в неравенство

$$
\begin{aligned}
\int_{I^{h}}\left[\left(u^{1}\right)^{2}\right. & \left.+h^{2}\left(u^{2}\right)^{2}+h^{2}\left(u^{3}\right)^{2}\right] d x \\
\leqslant & c \int_{I^{h}}\left[\left(\frac{\partial u^{1}}{\partial x_{1}}\right)^{2}+h^{2}\left(\frac{\partial u^{2}}{\partial x_{1}}+\frac{\partial u^{1}}{\partial x_{2}}\right)^{2}+h^{2}\left(\frac{\partial u^{1}}{\partial x_{3}}+\frac{\partial u^{3}}{\partial x_{1}}\right)^{2}\right. \\
& \left.+h^{4}\left(\frac{\partial u^{2}}{\partial x_{2}}\right)^{2}+h^{4}\left(\frac{\partial u^{2}}{\partial x_{3}}+\frac{\partial u^{3}}{\partial x_{2}}\right)^{2}+h^{4}\left(\frac{\partial u^{3}}{\partial x_{3}}\right)^{2}\right] d x
\end{aligned}
$$

из которого легко получить все утверждения леммы 17.1. 
Докажем неравенство (17.6) от противного. Допуская, что оно не выполняется, найдем $v_{h}$, для которых вьполнено (17.7) и

$$
\int_{I^{1}} v_{h} \cdot v_{h} d y=1, \quad \int_{I^{1}} e\left(v_{h}\right) \cdot e\left(v_{h}\right) d y \rightarrow 0 \text { при } h \rightarrow 0 .
$$

Из общего неравенства Корна (17.1) следует, что $v_{h}$ ограничена в $H^{1}\left(I^{1}\right)^{3}$.

Без потери обшности считаем, что

$$
\begin{gathered}
v_{h} \longrightarrow v_{0} \text { в } L^{2}\left(I^{1}\right), \quad v_{h} \rightarrow v_{0} \text { в } H^{1}\left(I^{1}\right), \\
e\left(v_{0}\right)=0, \quad \int_{I^{1}} v_{0} \cdot v_{0} d y=1 .
\end{gathered}
$$

Нетрудно понять (далее это будет строго доказано), что из условий (17.7) в пределе получаем

$$
0=\int_{K_{0}} v_{0} d y_{2} d y_{3}=\int_{K_{0}}\left(v_{0}^{2} y_{3}-v_{0}^{3} y_{2}\right) d y_{2} d y_{3}
$$

где $K_{0}=Q_{1}$ - единичный круг в плоскости $y_{2}, y_{3}$. Поскольку $v_{0}$ - жесткое перемещение (периодическое по $\left.y_{1}\right)$, то $v_{0}=c_{0}+t_{0}\left(0, y_{3},-y_{2}\right)$. Поэтому из (17.9) следует $v_{0}=0$, что противоречит (17.8).

Остается обосновать предельный переход в (17.7). Это не связано с теорией упругости, а относится к свойствам следа функций из классического соболевского пространства $H^{1}$. Для обоснования достаточно воспользоваться следующим предложением.

ПРЕДЛОЖЕНИЕ 17.2. Пусть $w_{h} \rightarrow w$ в $H^{1}\left(I^{1}\right), g$ - гладкая функиия. Тогда

$$
\lim _{h \rightarrow 0} \frac{1}{\left|K_{h}\right|} \int_{K_{h}} w_{h} g d y=\frac{1}{\left|K_{0}\right|} \int_{K_{0}} w g d y_{2} d y_{3},
$$

где $\left|K_{0}\right|-$ площадь круга $K_{0}$.

ДокАЗАТЕЛЬСТво. Сравним эллипсоид $K_{h}$ с тонкой пластиной $[-h, h] \times Q_{1}=$ $T_{h}$. Очевидно, эллипсоид $K_{h}$ содержится в пластине $T_{h}$, а их объемы имеют одинаковый порядок $h$ при $h \rightarrow 0$. Согласно теореме о следе имеем

$$
\int_{Q_{1}}\left|w\left(y_{1}, y_{2}, y_{3}\right)\right|^{2} d y_{2} d y_{3} \leqslant c(\delta) \int_{I^{1}}|w|^{2} d y+\delta \int_{I^{1}}|\nabla w|^{2} d y \quad \forall \delta>0 .
$$

Поэтому

$$
\frac{1}{\left|T_{h}\right|} \int_{T_{h}}|w|^{2} d y \leqslant c(\delta) \int_{I^{1}}|w|^{2} d y+\delta \int_{I^{1}}|\nabla w|^{2} d y \quad \forall \delta>0
$$

Такая же оценка верна и для эллипсоида $K_{h}$. Из нее с учетом сходимости $\int_{I^{1}}\left|w_{h}-w\right|^{2} d y \rightarrow 0$ и ограниченности $\nabla w_{h}$ в $L^{2}\left(I^{1}\right)$ получаем

$$
\begin{gathered}
\lim _{h \rightarrow 0} \frac{1}{\left|K_{h}\right|} \int_{K_{h}}\left|w-w_{h}\right|^{2} d y=0, \\
\lim _{h \rightarrow 0} \frac{1}{\left|K_{h}\right|} \int_{K_{h}} w_{h} g d y=\lim _{h \rightarrow 0} \frac{1}{\left|K_{h}\right|} \int_{K_{h}} w g d y .
\end{gathered}
$$


Очевидно, что для гладкой функции $w$

$$
\lim _{h \rightarrow 0} \frac{1}{\left|K_{h}\right|} \int_{K_{h}} w g d y=\frac{1}{\left|K_{0}\right|} \int_{K_{0}} w g d y_{2} d y_{3} .
$$

В общем случае это равенство также справедливо, и под $\left.w\right|_{K_{0}}$ следует понимать след функции $w \in H^{1}\left(I^{1}\right)$.

\section{§ 18. Приложение: вывод неклассического принципа усреднения методом асимптотических разложений}

Предположим, что для меры $\mu$ выполнено неравенство Корна (8.7), и поэтому справедливо представление (8.8). Поэтому можно рассмотреть периодическую задачу вида

$$
\operatorname{div}_{y}\left(A(y) e_{y}(w)\right)=-F_{0}(y)+\operatorname{div}_{y} F, \quad F_{0} \in L^{2}(\square, d \mu)^{N}, \quad F \in L^{2}(\square, d \mu)^{\frac{N(N+1)}{2}} .
$$

По определению

$w \in \mathcal{H}_{\mathrm{per}}^{1}, \quad w \perp \mathcal{R}, \int_{\square} A e_{y}(w) \cdot e_{y}(\varphi) d \mu=\int_{\square}\left(F_{0} \cdot \varphi+F \cdot e_{y}(\varphi)\right) d \mu \quad \forall \varphi \in C_{\mathrm{per}}^{\infty}(\square)^{N}$.

Легко понять, что необходимым и достаточным условием разрешимости служит условие ортогональности $F_{0} \perp \mathcal{R}$. При этом решение $w$ определено однозначно, поскольку оно нормировано условием $w \perp \mathcal{R}$.

С помощью представления (8.8) вспомогательная периодическая задача (8.4) принимает более привычную форму

$$
w \in \mathcal{H}_{\mathrm{per}}^{1}, \quad w \perp \mathcal{R}, \quad \operatorname{div}_{y}\left(A\left(\xi+e_{y}(w)\right)\right)=0,
$$

а формула (8.5) переписывается в виде

$$
A^{\text {hom }} \xi=\left\langle A\left(\xi+e_{y}(w)\right)\right\rangle .
$$

После этих замечаний обратимся к исходному уравнению (1.3), предполагая, что $f_{\varepsilon}=f \in C^{\infty}(\bar{\Omega})^{N}$. Приближение $\bar{u}_{\varepsilon}$ к точному решению $u_{\varepsilon}$ ищем в виде

$$
\bar{u}_{\varepsilon}(x)=u_{0}(x)+\chi(x, y)+\varepsilon u_{1}(x, y)+\varepsilon^{2} u_{2}(x, y)+\cdots,
$$

где $\chi, u_{1}, u_{2}$ периодичны по $y, y=\frac{x}{\varepsilon}$.

Вектор $\chi(x, y)$ будет выбран как линейная комбинация векторов

$$
\varphi(x) g(y), \quad \varphi \in C(\bar{\Omega}), \quad g \in \mathcal{R}_{1}
$$

(см. разложение (9.10)).

Предположим, что $u_{1}, u_{2}$ - гладкие функции аргумента $x \in \Omega$ со значениями в $\mathcal{H}_{\mathrm{per}}^{1}(\square, d \mu)$. Фактически эти функции будут иметь вид $\sum \varphi_{i}(x) b_{i}(y)$, где $\varphi_{i}-$ гладкие функции, $b_{i} \in \mathcal{H}^{1}(\square, d \mu)$. Для сложной функции $v\left(x, \frac{x}{\varepsilon}\right)$, где $v$ имеет указанный вид, используем формулу

$$
e(v)=e_{x}(v)+\varepsilon^{-1} e_{y}(v),
$$


в которой $e_{x}(v)$ - обычный тензор деформации, $e_{x}(v)=\frac{1}{2}\left\{\frac{\partial v_{i}}{\partial x_{j}}+\frac{\partial u_{j}}{\partial x_{i}}\right\}$, a $e_{y}(v)-$ некоторый (подлежащий определению) симметрический градиент вектор-функции $v(x, \cdot) \in \mathcal{H}_{\text {per }}^{1}$.

Для слагаемого $\chi$ градиент $e_{y}(\chi)$ выберем таким, чтобы выполнялось равенство $e(\chi)=0$ в $L^{2}\left(\Omega, d \mu_{\varepsilon}\right)$. Это можно сделать по лемме 7.2. Вектор-функции $u_{1}(x, \cdot)$, $u_{2}(x, \cdot)$ будут далее определены как решения некоторых периодических задач, что позволит сделать однозначный выбор их градиентов.

Итак, имеем разложения

$$
\begin{aligned}
e\left(\bar{u}_{\varepsilon}\right) & =e\left(u_{0}\right)+\varepsilon e\left(u_{1}\right)+\varepsilon^{2} e\left(u_{2}\right)+\cdots, \\
e\left(\bar{u}_{\varepsilon}\right) & =e\left(u_{0}\right)+e_{y}\left(u_{1}\right)+\varepsilon\left(e_{x}\left(u_{1}\right)+e_{y}\left(u_{2}\right)\right)+\cdots, \\
A e\left(\bar{u}_{\varepsilon}\right) & =A\left(e\left(u_{0}\right)+e_{y}\left(u_{1}\right)\right)+\varepsilon A\left(e_{x}\left(u_{1}\right)+e_{y}\left(u_{2}\right)\right)+\cdots,
\end{aligned}
$$

в которых слагаемое $\chi$ пока отсутствует.

Затем для симметрической матрищы $\Phi=\Phi(x, y), y=\varepsilon^{-1} x$, гладкой по $x$ и периодической по $y$, используем формулу

$$
\operatorname{div} \Phi=\operatorname{div}_{x} \Phi+\varepsilon^{-1} \operatorname{div}_{y} \Phi
$$

Здесь $\operatorname{div}_{x} \Phi-$ обычная дивергенция, $\operatorname{div}_{x} \Phi=\frac{\partial \Phi_{i j}}{\partial x_{j}}$, в то время как $\operatorname{div} \Phi-$ дивергенция в смысле меры $\mu_{\varepsilon}, \operatorname{a~div}_{y} \Phi$ - дивергенция в смысле меры $\mu$ при фиксированном $x$, т.е.

$$
\int_{\square} \Phi(x, y) e_{y}(\psi(y)) d \mu(y)=-\int_{\square} g(x, y) \psi(y) d \mu(y), \quad \psi \in C_{\mathrm{per}}^{\infty}(\square)^{N}, \quad g=\operatorname{div}_{y} \Phi .
$$

Таким образом, формулу (18.6) можно записать как тождество

$$
\int_{\Omega} \Phi \cdot e(\varphi) d \mu_{\varepsilon}=-\int_{\Omega} \operatorname{div}_{x} \Phi \cdot \varphi d \mu_{\varepsilon}-\varepsilon^{-1} \int_{\Omega} g \cdot \varphi d \mu_{\varepsilon}, \quad \varphi \in C_{0}^{\infty}(\Omega)^{N}
$$

в котором $\operatorname{div}_{x} \Phi=\frac{\partial \Phi_{i j}}{\partial x_{j}}, g=\operatorname{div}_{y} \Phi$ в смысле (18.7).

Это тождество легко проверяется для $\Phi=\eta(x) b(y)$, где $\eta \in C^{\infty}(\bar{\Omega}), \operatorname{div}_{y} b=a$ (в смысле меры $\mu$ ). Действительно, в этом случае $\operatorname{div}_{x} \Phi=b \nabla \eta$ и тождество (18.8) принимает вид (см. (3.9))

$$
\begin{aligned}
-\int_{\Omega} \eta b \cdot e(\varphi) d \mu_{\varepsilon} & =\int_{\Omega} b \nabla \eta \cdot \varphi d \mu_{\varepsilon}+\varepsilon^{-1} \int_{\Omega} \eta a \cdot \varphi d \mu_{\varepsilon} \\
& =\int_{\Omega} b \cdot(\nabla \eta \times \varphi) d \mu_{\varepsilon}+\varepsilon^{-1} \int_{\Omega} \eta a \cdot \varphi d \mu_{\varepsilon}
\end{aligned}
$$

Из соотношения $\operatorname{div}_{y} b=a$ имеем

$$
-\varepsilon^{-1} \int_{\Omega} a \cdot \eta \varphi d \mu_{\varepsilon}=\int_{\Omega} b \cdot e(\eta \varphi) d \mu_{\varepsilon}=\int_{\Omega} b \cdot \eta e(\varphi) d \mu_{\varepsilon}+\int_{\Omega} b \cdot(\nabla \eta \times \varphi) d \mu_{\varepsilon}
$$

и этим тождество (18.8) проверено. 
По формуле (18.6) из разложения (18.5) получим

$$
\begin{aligned}
\operatorname{div} A e\left(\bar{u}_{\varepsilon}\right)= & \varepsilon^{-1} \operatorname{div}_{y}\left(A\left(e_{x}\left(u_{0}\right)+e_{y}\left(u_{1}\right)\right)\right)+\operatorname{div}_{x}\left(A\left(e_{x}\left(u_{0}\right)+e_{y}\left(u_{1}\right)\right)\right) \\
& +\operatorname{div}_{y}\left(A\left(e_{x}\left(u_{1}\right)+e_{y}\left(u_{2}\right)\right)\right)+\cdots .
\end{aligned}
$$

Положим

$$
\operatorname{div}_{y}\left(A(y)\left(e_{x}\left(u_{0}\right)+e_{y}\left(u_{1}\right)\right)\right)=0 .
$$

Это - периодическая по $y$ задача, в которой $x$ - параметр. Видим, что $u_{1}(x, \cdot)$ есть решение задачи $(18.2)$ для $\xi=e_{x}\left(u_{0}(x)\right)$. Поэтому $u_{1}(x, y)=w\left(y, e_{x}\left(u_{0}(x)\right)\right)$, a из (18.3) следует равенство

$$
A^{\mathrm{hom}} e_{x}\left(u_{0}\right)=\left\langle A\left(e_{x}\left(u_{0}\right)+e_{y}\left(u_{1}\right)\right)\right\rangle .
$$

Рассмотрим выражение

$$
-\operatorname{div} A e\left(\bar{u}_{\varepsilon}\right)+\bar{u}_{\varepsilon}-f
$$

и приравняем нулю слагаемые порядка $\varepsilon^{0}$. Получим равенство

$$
-\operatorname{div}_{x}\left(A\left(e_{x}\left(u_{0}\right)+e_{y}\left(u_{1}\right)\right)\right)-\operatorname{div}_{y}\left(A\left(e_{x}\left(u_{1}\right)+e_{y}\left(u_{2}\right)\right)\right)+u_{0}+\chi=f,
$$

представляющее собой периодическую (по у) задачу нахождения вектор-функции $u_{2}=u_{2}(x, \cdot)$. Эта задача имеет вид (18.1), а критерий разрешимости $F_{0} \perp \mathcal{R}$ приводит к условию ортогональности

$$
-\operatorname{div}_{x}\left(A\left(e_{x}\left(u_{0}\right)+e_{y}\left(u_{1}\right)\right)\right)+u_{0}+\chi-f \perp \mathcal{R} \text { при каждом } x \in \Omega .
$$

Напомним, что пространство $\mathcal{R}$, помимо всех постоянных векторов, содержит множество $\mathcal{R}_{1}$, состоящее из периодических трансверсальных векторов.

Используя условие ортогональности (18.10), но только для постоянных векторов, получим

$$
-\operatorname{div}_{x}\left\langle A\left(e_{x}\left(u_{0}\right)+e_{y}\left(u_{1}\right)\right)\right\rangle+u_{0}+\langle\chi\rangle=f,
$$

или (см. формулу (18.3))

$$
-\operatorname{div} A^{\mathrm{hom}} e\left(u_{0}\right)+u_{0}+\langle\chi\rangle=f .
$$

Тем самым для нулевого приближения $u(x, y)=u_{0}(x)+\chi(x, y)$ выведено первое из двух соотношений $(2.6),(2.7)$, определяющих неклассическое усредненное уравнение. Покажем, как получается второе соотношение.

Мы знаем, что периодическая матрица $K(x, y)=A\left(e_{x}\left(u_{0}\right)+e_{y}\left(u_{1}\right)\right)$ тангенциальна при каждом $x \in \Omega$. Согласно лемме $5.2 K(x, y) \xi$ - тангенциальный периодический вектор (для любых $\xi \in \mathbb{R}^{N}, x \in \Omega$ ). Поэтому для $\varphi \in C_{0}^{\infty}(\Omega)$ вектор

$$
\int_{\Omega} \operatorname{div}_{x} K(x, y) \varphi(x) d x=-\int_{\Omega} K(x, y) \nabla \varphi(x) d x
$$

также тангенциален. Отсюда следует, что периодический вектор $\operatorname{div}_{x} K$ тангенциален при любом $x \in \Omega$; в частности, он ортогонален (поточечно) любому вектору из $\mathcal{R}_{1}$. 
Видим, что первое слагаемое в левой части (18.10) заведомо ортогонально множеству $\mathcal{R}_{1}$. Следовательно, $\left(u_{0}+\chi\right)-f=u-f \perp \mathcal{R}_{1}$. Получили соотношение $(2.7)$ и тем самым неклассический принцип усреднения.

Итак, порядок определения вектор-функций $u_{0}, \chi, u_{1}, u_{2}$ следующий.

Векторы $u_{0}$ и $\chi$ находим из (2.9). Видим, что $\chi$ действительно имеет структуpy (18.4). После этого определяем $u_{1}$ и $u_{2}$ как решения указанных выше периодических задач.

Приведенный вывод неклассического усредненного уравнения можно сделать совершенно строгим, иначе говоря, доказать соотношение (2.4). Но мы не будем останавливаться на этом.

\section{Список литературы}

1. Бахвалов Н. С., Панасенко Г. П. Осреднение процессов в периодических средах. М.: Наука, 1984.

2. ЖКков С. М., Козлов С. М., Олейник О. А. Усреднение дифференциальных операторов. М.: Наука, 1993.

3. Олейник О.А., Иосифьян Г. А., Шамаев А. С. Математические задачи теории сильно неоднородных упругих сред. М.: Изд-во МГУ, 1990.

4. Braides A., Defranceschi A. Homogenization of Multiple Integrals // Oxford Lecture Series in Mathematics and its Applications. V. 12. Oxford: Clarendon Press, 1998.

5. Saint Jean Paulin J., Cioranescu D. Homogenization of Reticulated Structures // Applied Mathematical Sciences. V. 136. Berlin-N. Y.: Springer-Verlag, 1999.

6. Sanchez-Palencia E. Homogenization Techniques for Composite Media. Berlin-N. Y.: Springer-Verlag, 1987.

7. ЖКиков В. В. Связность и усреднение. Примеры фрактальной проводимости // Матем. сб. 1996. Т. 187. № 8. С. 3-40.

8. Жиков В. В. К технике усреднения вариационных задач // Функц. анализ и его прилож. 1999. T. 33. № 1. C. 14-29.

9. Ciarlet P. G. Mathematical Elasticity. V. II. Theory of Plates // Studies in Mathematics and Applications. V. 27. Amsterdam-Lausanne-New York-Oxford-Shannon-Tokyo: Elsevier, 1997.

10. Acerbi E., Butazzo G., Percivale D. Thin inclusion in linear elasticity: a variational approach // J. reine angew. Math. 1988. V. 386. P. 99-115.

11. Kohn R. V., Vogelius $M$. A new model for thin plates with rapidly varying thikness. II. A convergence proof // Quart. Appl. Math. 1985. V. 43. P. 1-22.

12. Mosco U. Composite Media abd Asymptotic Dirichlet Forms // J. Funct. Anal. 1994. V. 123. P. 368-421.

13. Nazarov S. A. Korn's inequalities for functions of spatial bodies and thin rods // Math. Methods Appl. Sci. 1997. V. 20. № 3. P. 219-243.

14. Bouchitté G., Butazzo G., Seppecher P. Energies with respect to a measure and applications to low dimensional structures // Calc. Var. 1997. V. 5. P. 55-87.

15. Allaire G. Homogenization and two-scale convergence // SIAM J. Math. Anal. 1992. V. 23. P. 1482-1518.

16. Жиков В. В. Об одном расширении и применении метода двухмасштабной сходимости // Матем. сб. 2000. Т. 191. № 7. С. 31-72.

17. Жиков В. В. О весовых соболевских пространствах // Матем. сб. 1998. Т. 189. №8. C. $27-58$. 